Subwavelength metal nanogap surface for surface-enhanced Raman spectroscopy

Le Thi Ngoc Loan 
The research described in this thesis was carried out in the BIOS/Lab-on-a-Chip group at the MESA+ Institute for Nanotechnology, University of Twente, Enschede, The Netherlands. The project was financially supported by the Vietnamese Overseas Scholarship Program (Project number 322).

Committee members:

\section{Chairman}

Prof. dr. ir. P.M.G. Apers $\quad$ University of Twente

\section{Promotor}

Prof. dr. ir. A. van den Berg University of Twente

\section{Assistant promotor}

Dr. E.T. Carlen

University of Twente and

University of Tsukuba

\section{Members}

Prof. dr. J. Herek

University of Twente

Prof. dr. J. G. E. Gardeniers

University of Twente

Prof. dr. ir. R. P. H. Bischoff

Rijksuniversiteit Groningen

Prof. dr. J. Popp

Friedrich Schiller University

Title: Subwavelength metal nanogap surface for surface-enhanced Raman spectroscopy

Author: Le Thi Ngoc Loan

ISBN: 978-90-365-3861-9

DOI: $10.3990 / 1.9789036538619$

Publisher: Gildeprint, Enschede, The Netherlands

Copyright (C) 2015 by Le Thi Ngoc Loan, Enschede, The Netherlands 


\title{
Subwavelength metal nanogap surface for surface-enhanced Raman spectroscopy
}

\section{DISSERTATION}

\author{
To obtain \\ the degree of doctor at the University of Twente \\ on the authority of the rector magnificus, \\ prof. dr. H. Brinksma, \\ on account of the decision of the graduation committee, \\ to be publicly defended \\ on Friday the $22^{\text {nd }}$ of May 2015 at 16:45 hrs
}

by

Le Thi Ngoc Loan

born on March 13, 1980

in Binh dinh, Vietnam 
Dit proefschrift is goedgekeurd door

Promotor: Prof. dr. ir. Albert van den Berg

Assistant promotor: Dr. Edwin T. Carlen 
To my family 





\section{Contents}

CHAPTER 1 9

1.1 Surface-enhanced Raman spectroscopy 9

1.2 Thesis outline 12

$\begin{array}{ll}\text { CHAPTER 2 } & 15\end{array}$

2.1 Vibrational spectroscopy 16

$\begin{array}{ll}2.2 \text { Raman spectroscopy } & 17\end{array}$

2.3 Surface-enhanced Raman spectroscopy 23

2.4 SERS as a "standard" analytical technique 30

$\begin{array}{ll}\text { CHAPTER } 3 & 37\end{array}$

3.1 What makes a good SERS substrate? 38

3.2 Metal nanowire arrays 38

3.3 Self-forming metal nanowire array surfaces 40

3.4 Surface characterization $\quad 41$

3.5 Conclusions 44

$\begin{array}{lr}\text { CHAPTER } 4 & 47\end{array}$

4.1 Plasmonics and plasmons 48

4.2 Optical properties of gold and silver 52

4.3 Plasmonic substrates $\quad 54$

4.4 Reflectance spectroscopy

4.5 Surface-enhanced Raman spectroscopy 60

4.6 Conclusions 63

$\begin{array}{lr}\text { CHAPTER 5 } & 67\end{array}$

5.1 Introduction to plasmon-enhanced photoluminescence 68

5.2 Optical and electronic properties of gold 69

5.3 Plasmon-modulated photoluminescence of gold 74

5.4 Plasmon-enhanced photoluminescence and SERS 83

5.5 Conclusions $\quad 86$ 
CHAPTER $6 \quad 91$

6.1 Introduction to spectroelectrochemistry 92

6.2 Small volume SEC system 93

6.3 SEC analysis with metalloporphyrin modified electrodes 95

$\begin{array}{ll}6.4 \text { Conclusions } & 99\end{array}$

$\begin{array}{ll}\text { CHAPTER } 7 & 103\end{array}$

$\begin{array}{ll}7.1 \text { Conclusions } & 103\end{array}$

$\begin{array}{ll}7.2 \text { Recommendations } & 108\end{array}$

$\begin{array}{ll}\text { SUMMARY } & 111\end{array}$

$\begin{array}{ll}\text { SAMENVATTEN } & 113\end{array}$

$\begin{array}{ll}\text { LIST OF PUBLICATIONS } & 115\end{array}$

$\begin{array}{ll}\text { ACKNOWLEDGEMENTS } & 117\end{array}$ 


\section{Chapter 1}

Introduction

\subsection{Surface-enhanced Raman spectroscopy}

Over the last 40 years since its discovery, surface-enhanced Raman spectroscopy continues to emerge as an analytical technique used in various fields, such as biochemistry, biology, chemistry, and materials science. Surface-enhanced Raman spectroscopy is an ultrasensitive analytical technique that includes all of the important attributes of Raman spectroscopy; for example, it provides molecular structural information, as well as surface conformations of adsorbates on metal surfaces. Surface-enhanced Raman spectroscopy is based on surfaceenhanced Raman scattering (SERS) of molecules in close proximity to metal nanostructured surfaces. The dominant enhancement mechanism of SERS is an electromagnetic field enhancement effect, which is based on extremely large electric fields generated by localized surface plasmon resonances (LSPR) created in nanogaps located between metal nanostructures.

A good SERS substrate is one that provides large uniform and reproducible SERS electromagnetic field enhancements, which is key in order for surface-enhanced Raman spectroscopy to develop into a standard analytical technique. In this dissertation, a new topdown nanofabrication technology is presented to realize large area metal nanowire arrays with tunable sub-20 nm separation nanogaps without the use of chemical etching or milling of the metal layer; in this case gold and silver are used. The metal nanowire array nanofabrication technology is based on a self-regulating metal deposition procedure that is facilitated by closely spaced and isolated heterogeneous template surfaces that confine the metal deposition into two dimensions, and therefore, electrically isolated parallel arrays of metal nanowire can be realized with uniform and controllable nanogaps. Gold and silver nanowire arrays are presented with high-density on the order of $10^{5} \mathrm{~cm}^{-1}$, variable diameters down to $50 \mathrm{~nm}$, variable nanogaps down to $5 \mathrm{~nm}$, and very large nanogap length density on the order of $1 \mathrm{~km}$ $\mathrm{cm}^{-2}$. A significant achievement from this structure is that the coupled-mode LSPR wavelength in the nanogaps between adjacent nanowire array dimers can be precisely tuned to match any excitation source in the range from $500 \mathrm{~nm}$ up to approximately $1000 \mathrm{~nm}$, with a spatially averaged SERS analytical enhancement factor in the range of $10^{7}$ to $10^{8}$. Figure 1.1 
depicts measurements from a benzenethiol self-assembled monolayer (SAM) chemisorbed on a gold metal nanowire array substrate with LSPR wavelength matched to a He-Ne laser source. ${ }^{1}$

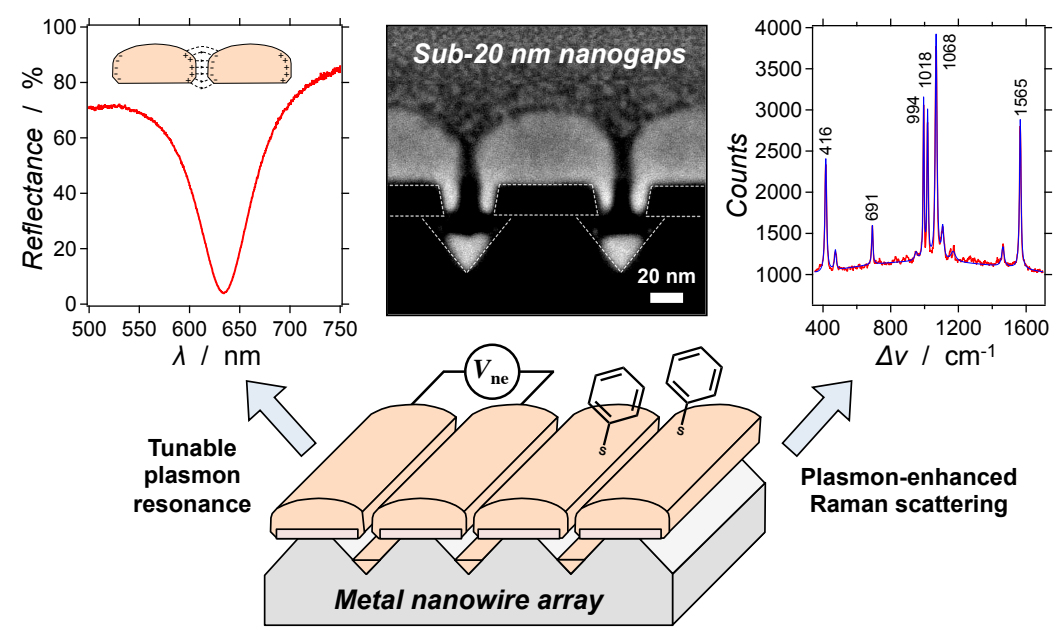

Figure 1.1. Gold nanowire array surfaces developed in this research provide highly uniform arrays of sub-20 nm nanogaps that form a dense array of LSPR structures that are used for surface-enhanced Raman spectroscopy.

Silver nanowire array surfaces have been used as a Raman surface spectroscopy tool to study the surface conformation characteristics of amine terminated alkanethiol SAMs. ${ }^{2}$ The surface conformation has implications for amine group availability, which is important when SAMs are used as linking layers for the binding of secondary molecules, which is particularly important for biosensing and medical diagnostics applications.

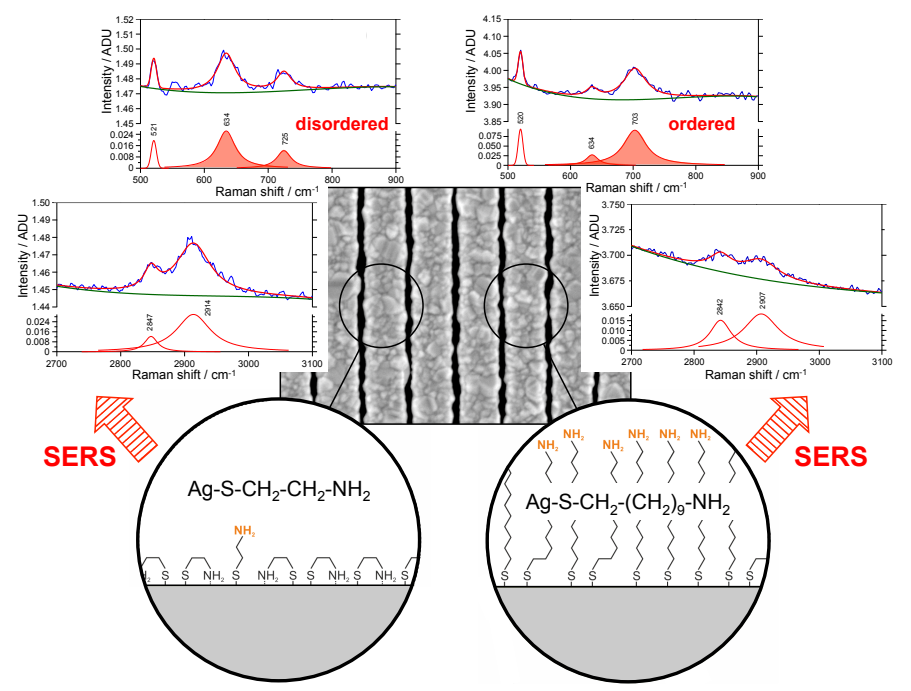

Figure 1.2. Silver nanowire array surfaces used for Raman surface spectroscopy to study the surface conformation characteristics of amine terminated alkanethiol SAMs.

\footnotetext{
${ }^{1}$ L. Le Thi Ngoc, et al. "Large area metal nanowire arrays with tunable sub-20 nm nanogaps," ACS Nano, 7, 5223, 2013.

2 J. Wiedemair, L. Le Thi Ngoc, et al. "Surface-enhanced Raman spectroscopy of self-assembled monolayer conformation and spatial uniformity on silver surfaces," J. Phys. Chem. C 118, 11857, 2014.
} 
Two different alkanethiols with different alkane chain lengths, namely 2-aminoethanethiol and 11-aminoundecanethiol, were investigated. It has been shown that both alkanethiols bind selectively to silver surfaces forming corresponding silver-thiolates, however surface conformations, which are related to the ordering of the SAM, are different for these two SAM systems. The quantification of the integrated intensity ratio of the trans to gauche $\mathrm{C}-\mathrm{S}$ stretch vibrations is used to assess the degree of SAM ordering.

Based on the observation of a broad background continuum accompanying all SERS spectra, since its discovery over 40 years ago, plasmon-modulated photoluminescence properties of the gold nanowire array surfaces were investigated. Two distinct single-photon plasmon-modulated photoluminescence processes are found to be generated from the gold nanowire array surfaces by tuning the spectral overlap of the incident laser source, localized surface plasmon resonance band, and the interband transitions between the $d$ and $s p$ bands, near the $X$ - and $L$-symmetry points of the electronic band structure of gold. ${ }^{3}$

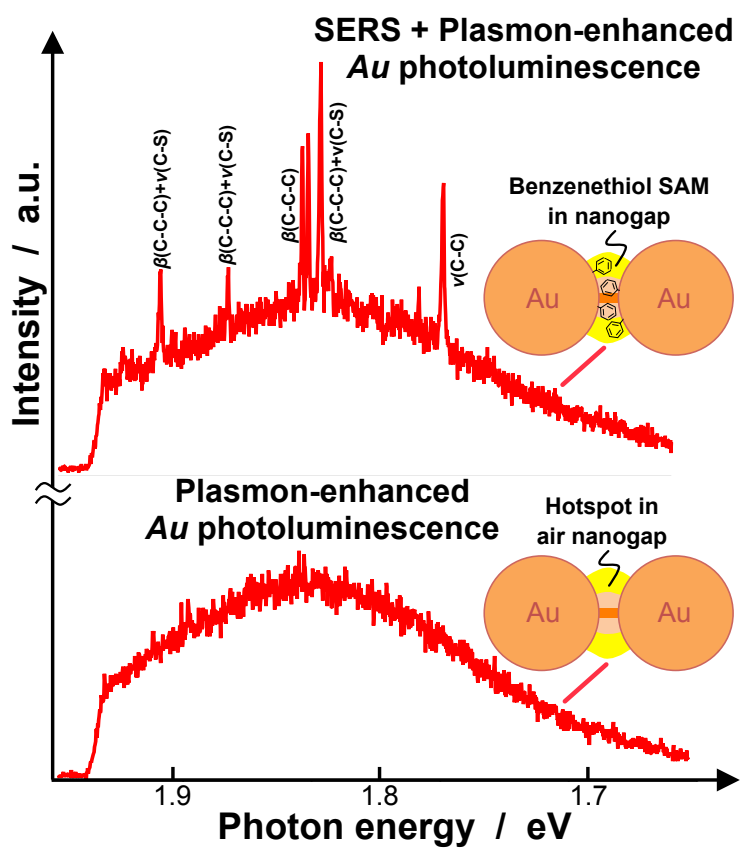

Figure 1.3. The broad background accompanying SERS spectra from benzenethiol and SAMs chemisorbed on gold nanowire array surfaces is shown to originate from plasmon-modulated photoluminescence.

The background continuum accompanying SERS spectra from benzenethiol and 4mercaptopyridine SAMs chemisorbed on the gold nanowire array surfaces is shown to originate from plasmon-modulated photoluminescence using a laser energy that is located near the $X$-symmetry point, in this case is a He-Ne laser.

An integrated SERS spectroelectrochemical (SEC) analysis system has been developed

\footnotetext{
${ }^{3}$ L. Le Thi Ngoc, et al. "Plasmon-modulated photoluminescence from gold nanostructures and its dependence on plasmon resonance, excitation energy, and band structure," Opt. Express 23, 5547, 2015.
} 
that combines a small volume microfluidic sample chamber with a compact three-electrode configuration for in situ surface-enhanced Raman spectroelectrochemistry. The SEC system includes a gold nanowire array surface that serves dual roles as the electrochemical working electrode and a SERS-active surface, a microfabricated platinum counter electrode, and an external $\mathrm{Ag} / \mathrm{AgCl}$ reference electrode.

The gold nanowire working electrode surface enables highly sensitive in situ surfaceenhanced Raman spectroscopy through large and reproducible SERS enhancements, which eliminates the need for resonant wavelength matching of the laser excitation source with the electronic absorption of the target molecule. The new SEC analysis system has the merits of applicability to wide range of target molecules, small sample volume, and low detection limit. In situ SERS spectroelectrochemistry measurements of the metalloporphyrin hemin are demonstrated showing shifts of the oxidation marker band of the iron core with the gold nanowire working electrode under potential control. ${ }^{4}$

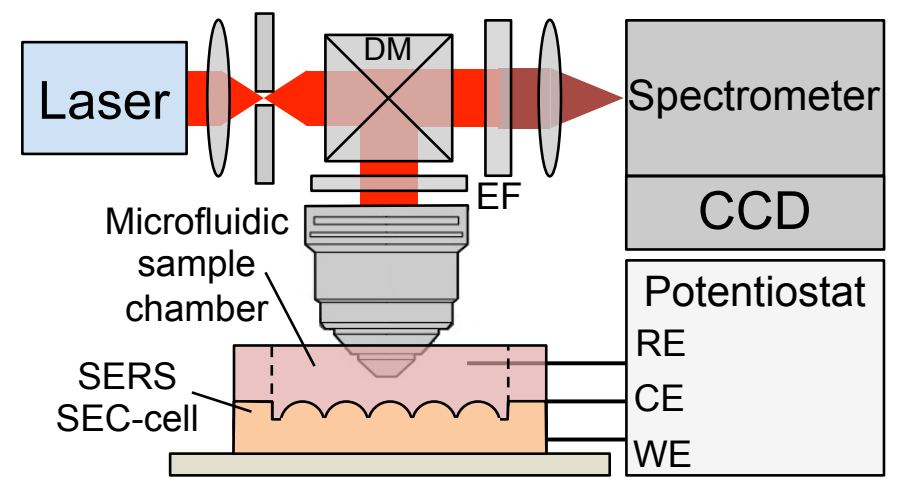

Figure 1.4. Integrated SERS spectroelectrochemical analysis system has been developed that combines a small volume microfluidic sample chamber with a compact three-electrode configuration for in situ surface-enhanced Raman spectroelectrochemistry.

\subsection{Thesis outline}

In chapter 2, a brief introduction to conventional Raman spectroscopy is described in terms of electromagnetic radiation and classical light scattering. Surface-enhanced Raman spectroscopy is described with respect to conventional Raman spectroscopy; SERS is described with respect to the molecular scattering cross section tensor. A short introduction to SERS surface selection rules is presented.

In Chapter 3, the fabrication process of the metal nanowire array surfaces is presented, along with a description of their characterization using high-resolution scanning electron microscopy and atomic force microscopy. An empirical model is introduced to estimate the gap size based on the pitch of a nanopatterned silicon nitride template and thickness of

\footnotetext{
${ }^{4}$ T. Yuan, L. Le Thi Ngoc, et al. "In situ surface-enhanced Raman spectroelectrochemical analysis system with a hemin modified nanostructured gold surface," Anal. Chem. 87, 2588, 2015.
} 
deposited metal.

In Chapter 4, the optical characterization of gold and silver nanowire array surfaces using reflection spectroscopy and surface-enhanced Raman spectroscopy is presented. Reflection spectroscopy is used to demonstrate the plasmonic tunability and uniformity of the dimensions of the metal nanowire array surfaces with normally incident linearly polarized white light aligned perpendicular to the nanogaps. Surface-enhanced Raman spectroscopy is used to assess the characteristics of the SERS enhancements of the gold nanowire array surfaces by covering the surfaces with a benzenethiol SAM, which provides an estimation of the uniformity of the spatially averaged enhancement factor.

In Chapter 5, an overview of conventional photoluminescence from polycrystalline gold layers and plasmon-modulated photoluminescence from gold nanostructures is first presented, followed by a systematic study of plasmon-modulated photoluminescence from the gold nanowire array surfaces presented in Chapter 3. The study outlines the importance of the spectral overlap of the excitation and surface plasmon resonance energies with the interband transition of the electronic band structure of gold. In addition, it is shown that plasmonenhanced photoluminescence is the main source of the background continuum observed in SERS spectra from surface adsorbed molecules on gold nanostructures with a red laser.

In Chapter 6, an integrated in situ SERS spectroelectrochemical analysis system is presented that combines a small volume microfluidic sample chamber with a compact threeelectrode configuration, which includes a gold nanowire array surface that serves dual roles as an electrochemical working electrode and as a substrate for SERS, a microfabricated platinum counter electrode, and an external $\mathrm{Ag} / \mathrm{AgCl}$ reference electrode. The gold nanowire working electrode surface enables in situ SERS spectroscopy measurements from small numbers of molecules through large and reproducible SERS enhancements, which eliminates the need for resonant wavelength matching of the laser excitation source with the electronic absorption of the target molecule as required by surface-enhanced resonance Raman scattering. Preliminary in situ SERS spectroelectrochemistry measurements are presented of the metalloporphyrin hemin showing shifts of the oxidation marker band of the iron core.

In Chapter 7, the conclusions of the work described in this thesis are summarized, and recommendations are provided to improve the reported results. 



\section{Chapter 2}

\section{Surface-enhanced Raman spectroscopy ${ }^{1}$}

Raman scattering is first introduced using a quantum mechanical model and followed by a description in terms of electromagnetic radiation and classical light scattering. Surface-enhanced Raman scattering is then described in terms of classical Raman scattering with respect to the molecular scattering cross section tensor. A short introduction of surface selection rules for surface-enhanced Raman scattering is described.

\footnotetext{
${ }^{1}$ Select sections adapted from: J. Wiedemair, L. Le Thi Ngoc, A. van den Berg, and E.T. Carlen, "Surfaceenhanced Raman spectroscopy of self-assembled monolayer conformation and spatial uniformity on silver surfaces," J. Phys. Chem. C 118, 11857, 2014.
} 


\subsection{Vibrational spectroscopy}

Spectroscopy is based on the interaction of electromagnetic radiation with matter. The molecular constituents of matter consist of electrically charged nuclei and electrons that can interact with electromagnetic fields and in certain cases absorb the energy carried by the incident electromagnetic radiation. Figure 2.1 shows several different molecular processes that occur depending on the molecule and the energy of the incident radiation. The right side of the energy diagram illustrates direct absorption processes. Fluorescence occurs when the incident radiation has an energy large enough to excite electrons of the molecule into an excited electronic state, for example, the transition from the ground state to an excited electronic state $s=0 \rightarrow s^{\prime}=2$ by the absorption of the incident photon energy.

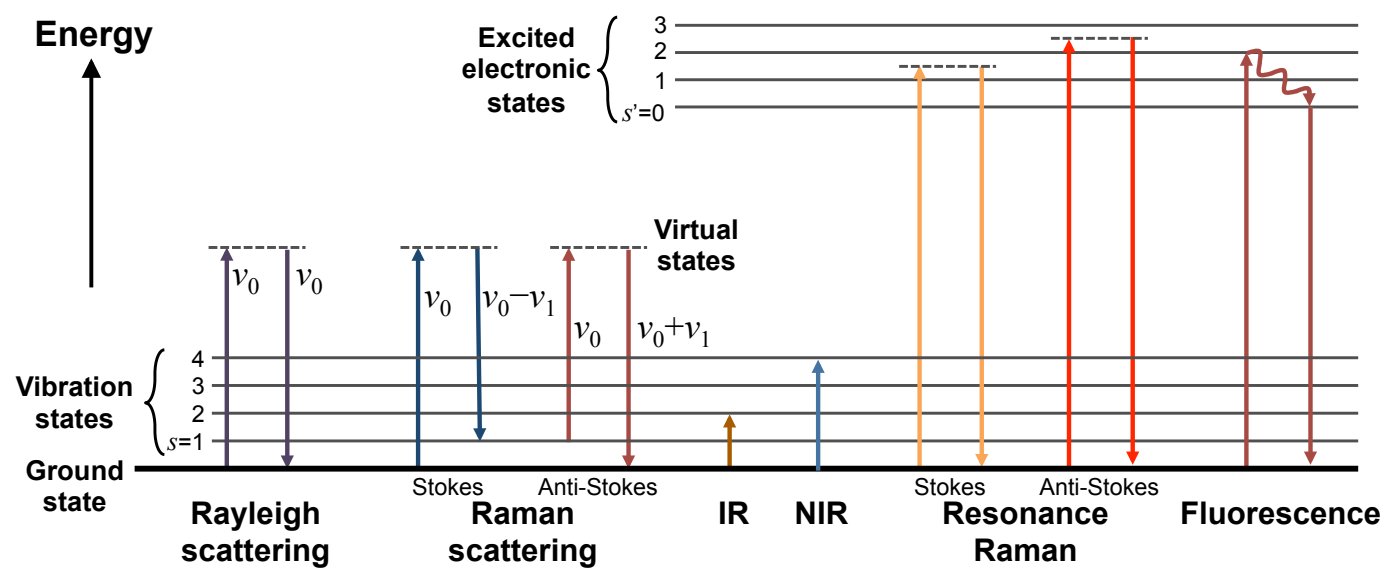

Figure 2.1. Energy-level diagram for comparing different optical processes occurring in materials for incident radiation ranging from visible to infrared wavelengths.

Electrons in the excited state decay to the lowest energy level $s^{\prime}=0$ via non-radiative transitions, and then spontaneously transition back to the ground state energy and emit radiation. Resonance Raman scattering occurs when the incident energy overlaps with the electronic absorption band of the molecule and results in an extremely strong Raman band intensity that originates from the electronic transition. The lifetime of the excited state of a typical resonance Raman process is on the order of $10^{-14} \mathrm{~s}$, while the lifetime of a fluorescence process is in the range of $10^{-5} \mathrm{~s}$ to $10^{-8} \mathrm{~s}$ [1]. Infrared (IR) and near-infrared (NIR) absorption, for example, transitions from the ground state to vibration states $s_{2}$ and $s_{4}$, respectively, arises from a direct resonance between the frequency of the incident radiation and the vibration frequency of a particular normal mode of vibration. The resonant interaction of the molecule with the incident radiation requires a change in the dipole moment of the molecule with respect to the vibrational motion. IR absorption is a one-photon event, where the photon is annihilated, and the molecule is elevated in vibrational energy by the photon energy at the frequency of the vibrational resonance. 
The left side of the energy diagram shows scattering processes. In scattering processes, the interaction with incident radiation excites the molecule from the ground state to a virtual energy state, indicated with a dashed line, which is well below the excited electronic state energy of the molecule. A virtual state is a short lifetime distortion of the electron distribution by the electric field of the incident radiation. Rayleigh scattering corresponds to the light scattered at the frequency of the incident radiation, in this case $v_{0}$, which is also referred to as elastically scattered radiation. Raman scattering involves scattered radiation shifted in frequency, thus energy, from the frequency of the incident radiation by the vibrational energy that is lost or gained in the molecule, which is also referred to as inelastic scattering, in this case $v_{0} \pm v_{\mathrm{s}}$ depending on the initial energy of the molecule. If the molecule gains vibrational energy, with frequency $v_{0}-v_{\mathrm{s}}$, the scattering is called Stokes Raman scattering, whereas if the molecule loses vibrational energy by starting from an elevated vibrational level, with frequency $v_{0}+v_{\mathrm{s}}$, the process is known as anti-Stokes Raman scattering. Rayleigh and Raman scattering are two photon processes. The two-photon events are associated with a single coherent quantum mechanical process, which makes Rayleigh and Raman scattering fundamentally different from the one-photon absorption process. Raman scattering involves the change in the polarizability of the molecule with respect to its vibrational motion. The interaction of the polarizability with the incident radiation creates an induced dipole moment in the molecule, and the radiation emitted by the induced dipole moment contains the observed Raman scattered spectra. The total radiation scattered by the induced dipole moment of the molecule consists of both Rayleigh scattered and Raman scattered light. Raman scattering and IR absorption are the basic mechanisms that are used to obtain information about the structure and properties of molecules from their vibrational transitions.

\subsection{Raman spectroscopy}

In 1905 Einstein proposed the hypothesis of light quanta. In 1923, Smekal postulated that light has a quantum structure and predicted that scattered monochromatic light is comprised of elastically and inelastically scattered radiation. Five years later, in 1928, Krishna and Raman first reported spectra showing the shift of frequency of scattered light by liquid benzene, which was subsequently called the Raman effect [2]. In this effect, Raman scattered light, is shifted in frequency with respect to the incident radiation, which is independent from the frequency of the incident light and is a unique property of scattering. Raman spectroscopy is a fundamental form of molecular spectroscopy, which is based on Raman scattering and provides information about chemical structure, molecular interactions, and molecular conformations. A Raman scattering spectrum provides a number of bands that represent the 
intensity and frequency position of the inelastic scattered light, where each band corresponds to the vibration of a specific molecular bond. Figure 2.2 shows an example of a Raman scattering spectrum showing Stokes, anti-Stokes and Rayleigh scattered bands [3].

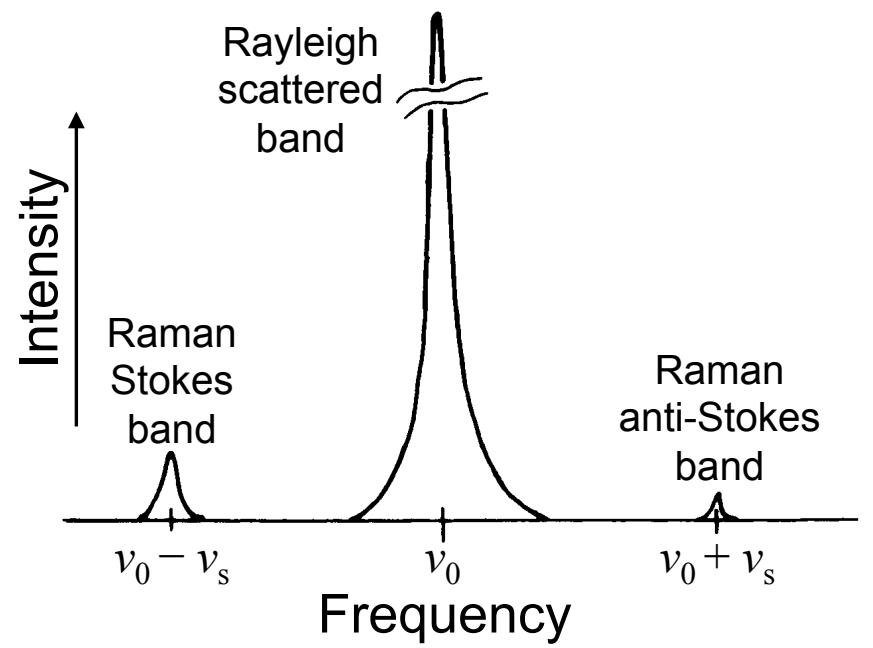

Figure 2.2. Example of Raman and Rayleigh scattered bands from excitation at frequency $v_{0}$ and molecular vibration frequency $v_{\mathrm{s}}[3]$.

The energy difference between the incident radiation $h v_{0}$ and the Raman scattered radiation $h v_{\mathrm{s}}$, is called the Raman shift, and provides information about vibration frequency of the particular vibration mode of the molecule [4].

Table 2.1. IR and Raman frequencies and relative intensities of selected organic compounds [4]. $\mathrm{vs} \equiv \mathrm{very}$ strong, $\mathrm{s} \equiv$ strong, $\mathrm{m} \equiv$ medium, $\mathrm{w} \equiv \mathrm{weak}$, and dash $(-) \equiv \mathrm{absent}$

\begin{tabular}{|c|c|c|c|}
\hline Vibration & $\begin{array}{c}\text { Frequencies } \\
\left(\mathrm{cm}^{-1}\right)\end{array}$ & $\begin{array}{c}\text { IR } \\
\text { intensity }\end{array}$ & $\begin{array}{c}\text { Raman } \\
\text { intensity }\end{array}$ \\
\hline Benzene ring breathing & 992 & - & vs \\
\hline Alcohol O-H stretch & $3635-3644$ & $\mathrm{~m}$ & $\mathrm{w}$ \\
\hline Acetylene $\mathrm{C}-\mathrm{H}$ bend & $825-640$ & $\mathrm{~s}$ & $\mathrm{w}$ \\
\hline Acetylene $\mathrm{C} \equiv \mathrm{C}$ & $2230-2237$ & - & $\mathrm{s}$ \\
\hline $\mathrm{C} \equiv \mathrm{N}$ stretch in $\mathrm{R}-\mathrm{CN}$ & $2230-2250$ & $\mathrm{~s}$ & vs \\
\hline Cyanate $\mathrm{C} \equiv \mathrm{N}$ & $2245-2256$ & $\mathrm{~s}$ & vs \\
\hline $\mathrm{C}-\mathrm{H}$ in $\mathrm{R}-\mathrm{CHO}$ & $2800-2850$ & $\mathrm{~m}$ & - \\
\hline $\mathrm{C}=\mathrm{O}$ in $\mathrm{R}-\mathrm{CHO}$ & $1730-1740$ & vs & $\mathrm{w}$ \\
\hline $\mathrm{R}-\mathrm{NO}_{2}$ asymmetric stretch & $1530-1600$ & vs & $\mathrm{m}-\mathrm{w}$ \\
\hline $\mathrm{R}-\mathrm{NO}_{2}$ symmetric stretch & $580-704$ & $\mathrm{~s}$ & vs \\
\hline C-S stretch & $1310-1397$ & - & vs \\
\hline S-H stretch & $2560-2590$ & $\mathrm{~W}$ & $\mathrm{~s}$ \\
\hline
\end{tabular}

IR spectroscopy is an important molecular vibration spectroscopy, which manifests from absorption and a direct resonance between the incident radiation and the vibrational frequency of a particular mode of vibration, as previously described. Although Raman and IR spectroscopies are both based on vibrational modes of the molecule, they provide similar, but complementary information. IR spectroscopy is based on the IR absorption process and directly probes vibrational transitions, whereas Raman spectroscopy is based on an inelastic scattering process. Molecular vibrations can be Raman-active, IR-active, or both. Table 2.1 
lists frequencies of a few select compounds with relative intensities of both processes for different types of molecular bonds [4]. Symmetric modes, double or triple bonds, covalent bonds, and homonuclear molecules usually have strong Raman intensities, hence are Ramanactive. Both IR and Raman spectra can show the same vibrational frequencies of the same molecular vibrational modes. These differences can be interpreted by the specific selection rules. Nevertheless, they are complementary techniques having distinct advantages and disadvantages. Table 2.2 compares some important properties of Raman and IR spectroscopies.

Table 2.2. General properties of Raman and IR spectroscopies [4].

\begin{tabular}{|c|c|c|}
\hline Property & Raman spectroscopy & IR spectroscopy \\
\hline Selection rules & Change in molecular polarizability & Change in dipole moment \\
\hline Physical process & Raman scattering $\rightarrow$ weak process & Absorption $\rightarrow$ strong process \\
\hline Information & $\begin{array}{l}\text { Probes molecular vibration and } \\
\text { structure }\end{array}$ & $\begin{array}{l}\text { Probes molecular vibration } \\
\text { and structure }\end{array}$ \\
\hline Measurements & $\begin{array}{l}\text { - Small interference from water, } \\
\text { glass tubing or polymer } \\
\text { - Stronger in UV-VIS (factor of } v^{4} \text { ) }\end{array}$ & $\begin{array}{l}\text {-Water and glass strongly } \\
\text { absorbed by IR radiation } \\
\text { - Infrared and near infrared }\end{array}$ \\
\hline $\begin{array}{l}\text { Sample } \\
\text { preparation }\end{array}$ & $\begin{array}{l}\text { - No sample preparation needed } \\
\text { - Small sample volume (on the } \\
\text { order of the laser spot size) } \\
\text { - Non-destructive }\end{array}$ & $\begin{array}{l}\text {-Sample preparation needed } \\
\text {-Large sample volume } \\
\text {-Destructive }\end{array}$ \\
\hline Techniques & $\begin{array}{l}\text { Raman scattered light can be } \\
\text { transmitted over long distances } \\
\text { (remote analysis) }\end{array}$ & Remote analysis not possible \\
\hline Instrumentation & $\begin{array}{l}\text { - Laser and highly sensitive } \\
\text { collection system required } \\
\text { - Interference by impurity } \\
\text { fluorescence } \\
\text { - Sample can be destroyed by lasers }\end{array}$ & $\begin{array}{l}\text { - Lower cost instrumentation } \\
\text { - No lasers needed } \\
\text { - No fluorescence interference }\end{array}$ \\
\hline
\end{tabular}

The use of Raman spectroscopy was initially limited since its discovery due to the weak intensity of the Raman scattering process, overlap with the fluorescence band, and inefficient light collection and detection methods. The growth of Raman spectroscopy as an analytical technique was triggered by the invention of the laser in 1958 and later with the use of resonance Raman scattering [5]. In the years that followed, the use of Raman spectroscopy further increased with improvements in instrumentation and the introduction of fiber optics. Nowadays, Raman spectroscopy has become an important analytical technique for various applications in materials science, and biomedical and chemical analyses. Raman spectroscopy can provide information about molecular symmetry of small molecules and functional groups in large and complex molecules, thus it is possible to identify molecular structure by using symmetry selection rules [6]. Since water is a weak Raman scatterer, Raman spectroscopy is well suited for studies of chemical and biological systems in aqueous samples. For example, 
iron porphyrins are very important model systems that serve as active sites of biological function of the heme protein, which is the topic in Chapter 6. The versatility of the iron core can take a variety of spin states (high, low and intermediate), oxidation states (II, III, IV, and V) and coordination numbers (four, five, and six). Using resonance Raman spectroscopy, Spiro and Strekas showed that the $v_{3}, v_{10}, v_{19}, v_{28}$ vibration modes are core-size sensitive in which $v_{3}$, near $1500 \mathrm{~cm}^{-1}$, can be used as a spin state marker; and the $v_{4}$ vibration band is used as an oxidation state marker, at $1350 \mathrm{~cm}^{-1}$ for $\mathrm{Fe}$ (II) and $1370 \mathrm{~cm}^{-1}$ for $\mathrm{Fe}$ (III) [7]. In Chapter 6 , the metalloporphyrin hemin is studied using non-resonant surface-enhanced Raman spectroelectrochemistry. With these advancements, Raman spectroscopy is gaining widespread use in materials characterization, defect analysis, chemical reaction monitoring, and disease diagnosis [3,4,7-9].

\subsubsection{Raman scattering}

Raman scattering can be described using models of classical light scattering and electromagnetic radiation, which are based on the interaction of vibrational and rotational motions of molecules in response to incident electromagnetic radiation [3]. When electromagnetic radiation is incident on a molecule, a force acts on the electron orbitals of the molecule $\boldsymbol{F}=Q \boldsymbol{E}$, where $\boldsymbol{F}$ is the force vector, $Q$ is the net charge, and $\boldsymbol{E}\left[\right.$ unit: $\left.V \mathrm{~m}^{-l}\right]$ is the electromagnetic field. This force induces an electric dipole moment $\boldsymbol{p}=Q \boldsymbol{d}$, where $\boldsymbol{p}$ [unit: $C$ $m]$ is the dipole moment and $\boldsymbol{d}$ is the displacement vector, which acts as a dipole emitter that radiates Rayleigh scattered light and Raman scattered light characteristic of the vibrational modes of the molecule [1]. The induced oscillating electric dipole vector of a molecule can be expressed as a classical scattering process

$$
P=\alpha \cdot E,
$$

where $\boldsymbol{P}\left[\right.$ unit: $\left.\mathrm{C} \mathrm{m}^{-2}\right]$ is the polarization ${ }^{2}$ and $\boldsymbol{\alpha}\left[\right.$ unit: $\left.\mathrm{m}^{-3} \mathrm{C}^{2} \mathrm{~kg}^{-1} \mathrm{~s}^{2}\right]$ is the polarizability tensor of the molecule. The components of the polarizability tensor contain two indices; one is associated with the incident photon and the other with the scattered photon. The time dependence of the electric field of the incident radiation with frequency $\boldsymbol{v}_{0}$ is $\boldsymbol{E}=\boldsymbol{E}_{0} \cos \left(2 \pi v_{0} t\right)$. We usually consider the normal vibration modes of the molecule with displacement

$$
d_{i}=d_{0 i} \cos \left(2 \pi v_{s i} t\right)
$$

where $d_{0 \mathrm{i}}$ is the amplitude and $v_{\mathrm{si}}$ is the fundamental harmonic frequency of the $i^{\text {th }}$ vibration

\footnotetext{
${ }^{2}$ The polarization is defined as $\boldsymbol{P}=N\langle\boldsymbol{p}\rangle$, where $N$ is the number of dipole moments per unit volume and $\langle\boldsymbol{p}\rangle$ is the average dipole moment.
} 
mode. For small displacements, the polarizability ${ }^{3}$ of the electrons in the molecule will be affected by the molecular vibration and can be represented as a Taylor expansion in $d_{\mathrm{i}}$

$$
\begin{aligned}
\alpha & \approx \alpha_{0}+\left(\partial \alpha / \partial d_{i}\right) d_{i}+\cdots \\
& =\alpha_{0}+\left(\partial \alpha / \partial d_{i}\right) d_{0 i} \cos \left(2 \pi v_{s i}\right)+\cdots
\end{aligned}
$$

Here, $\alpha_{0}$ is the polarizability at the equilibrium position, and $\partial \alpha / \partial d_{\mathrm{i}}$ is the change of $\alpha$ with respect to displacement change. Combining Eqs. (2.1)-(2.3) and retaining only the first order term of the expansion gives

$$
\begin{aligned}
& P \approx\left[\alpha_{0}+\left(\partial \alpha / \partial d_{i}\right) d_{0 i} \cos \left(2 \pi v_{s i} t\right)\right] E_{0} \cos \left(2 \pi v_{0} t\right) \\
& =\alpha_{0} E_{0} \cos \left(2 \pi v_{0} t\right)+\frac{1}{2} E_{0}\left(\frac{\partial \alpha}{\partial d_{i}}\right) d_{0 i}\left[\cos \left(2 \pi\left(v_{0}-v_{s i}\right) t\right)+\cos \left(2 \pi\left(v_{0}+v_{s i}\right) t\right)\right]
\end{aligned}
$$

Equation (2.4) indicates two distinct induced dipole frequency components: the first term results in Rayleigh scattered radiation, with frequency $v_{0}$, and the second term corresponds to Raman scattering, where the frequency $v_{0}-v_{\mathrm{s}}$ is the Stokes scattered radiation, and the frequency $v_{0}+v_{\mathrm{si}}$ is the anti-Stokes scattered radiation. A vibration mode is Raman-inactive if $\partial \alpha / \partial d_{\mathrm{i}}=0$, and Raman-active if $\partial \alpha / \partial d_{\mathrm{i}} \neq 0$ [4]. From this simple analysis, some useful characteristics should be highlighted [9]:

- The polarizability and intensity of Rayleigh and Raman scattering are linear with excitation laser power in the first order expansion

- Vibration modes with $\partial \alpha / \partial d_{\mathrm{i}} \neq 0$ are generally Raman-active

- Although not evident in the classical scattering derivation, $\partial \alpha / \partial d_{\mathrm{i}}$ can vary significantly among molecules and vibration modes, which can lead to variations in the Raman intensity

- $\partial \alpha / \partial d_{\mathrm{i}}$ is generally smaller than $\alpha_{0}$, thus Raman scattered radiation is much weaker than Rayleigh scattered radiation

The classical description of the Raman effect explains many of the experimental observations quite well; however, in the classical picture, equal intensities of the Stokes and anti-Stokes bands are expected, while, in fact, the Stokes bands are much stronger in intensity, which can only be explained using a quantum mechanics treatment of the Raman effect $[1,10]$. Since the population of molecules with energy at the ground state $s=0$ is much larger than with energy $s=1$ (Fig. 2.1), based on the Maxwell-Boltzmann distribution, the Stokes band intensities are stronger than the anti-Stokes band intensities under normal conditions [1]. The intensity ratio of the Stokes and anti-Stokes bands depends on the population of the first

\footnotetext{
${ }^{3}$ The indices of the polarizability tensor components are not shown.
} 
vibrationally excited states, which are related to temperature by

$$
\frac{I_{R}\left(v_{0}+v_{s i}\right)}{I_{R}\left(v_{0}-v_{s i}\right)}=\frac{\left(v_{0}+v_{s i}\right)^{4}}{\left(v_{0}-v_{s i}\right)^{4}} \exp \left(\frac{-h v_{s i}}{k T}\right),
$$

where $I_{\mathrm{R}}$ is the Raman intensity, $k$ is Boltzmann's constant and $T$ is temperature.

Today most spectrometers output a photon flux $\Phi_{\mathrm{R}}\left[\right.$ unit: photon $\left.s^{-1}\right]$, which can be represented as $\Phi_{R}=\Phi_{0} \sigma_{s}^{o} v_{0}\left(v_{0}-v_{s}\right)^{3} D d z=\Phi_{0} \sigma_{s} D d z$, where $\Phi_{0}$ is the laser flux, $\sigma_{\mathrm{s}}\left[\right.$ unit: $\mathrm{cm}^{2}$ molecule $\left.{ }^{-1}\right]$ is the integrated frequency dependent scattering cross section $\sigma_{s}=\sigma_{s}^{o} v_{0}\left(v_{0}-v_{s}\right)^{3}$ of the particular vibration mode, $D$ [unit: molecules $\mathrm{cm}^{-3}$ ] is the number density of scatterers, and $d z$ is the path length of the laser in the sample [4]. The observed flux, or intensity ${ }^{4}$, of Raman scattering scales with $v_{0}\left(v_{0}-v_{\mathrm{s}}\right)^{3}$, and is proportional to $\sigma_{\mathrm{s}}$, where $\sigma_{\mathrm{s}} \propto \partial \alpha / \partial d$ from Eq. (2.4) [1]. The integrated cross section $\sigma_{\mathrm{s}}$ is integrated over all directions of the sample, i.e. $4 \pi$ sr, and integrated over the wavelength range of the entire Raman band. In practice, it is more convenient to define the differential scattering cross section $\mathrm{d} \sigma_{\mathrm{s}} / \mathrm{d} \Omega$ [unit: $\mathrm{cm}^{2}$ molecule ${ }^{-1}$ $s r^{-1}$ ], where $\Omega$ represents the solid angle of the collection configuration [4]. The intensity is

$$
\Phi_{R}=\Phi_{0}\left(d \sigma_{S} / d \Omega\right) \kappa D d z
$$

where $\kappa$ is related to the collection efficiency. Table 2.3 lists some commonly reported cross section values.

\begin{tabular}{|c|c|c|}
\hline Sample, vibration band & $\begin{array}{c}\lambda_{0} \\
(\mathrm{~nm})\end{array}$ & $\begin{array}{c}\boldsymbol{d} \sigma_{\mathrm{s}} / \boldsymbol{d} \boldsymbol{\Omega}\left(\times 10^{30}\right) \\
\left(\mathrm{cm}^{2} \mathrm{molecule}^{-1} \mathrm{sr}^{-1}\right)\end{array}$ \\
\hline Benzene liquid, $992 \mathrm{~cm}^{-1}$ & 647 & 10.6 \\
\hline Benzene, neat, $992 \mathrm{~cm}^{-1}$ & 514.5 & 28.6 \\
\hline $\mathrm{N}_{2}$ gas, $2331 \mathrm{~cm}^{-1}$ & 514.5 & 0.43 \\
\hline $\mathrm{H}_{2} \mathrm{O}$ liquid, $1595 \mathrm{~cm}^{-1}$ & 514.5 & 0.11 \\
\hline Cyclohexane liquid, $802 \mathrm{~cm}^{-1}$ & 647 & 2.1 \\
\hline Rhodamine 6G, $1510 \mathrm{~cm}^{-1}$ & 632 & 2400 \\
\hline Pyridine, $1004 \mathrm{~cm}^{-1}$ & 514.5 & 11 \\
\hline Pyrazine, $1004 \mathrm{~cm}^{-1}$ & 514.5 & 16 \\
\hline
\end{tabular}

Table 2.3. Measured Raman cross sections [4,13].

The Raman cross section is typically very small, for example, the differential cross section of benzene is $\mathrm{d} \sigma_{\mathrm{s}} / \mathrm{d} \Omega \sim 10^{-29} \mathrm{~cm}^{2}$ molecule ${ }^{-1} \mathrm{sr}^{-1}$, considered a strong Raman scatterer, is about 13 orders of magnitude smaller than a typical fluorescence cross section [11,12]; this is one of the main limitations of Raman spectroscopy.

One of the main challenges of using conventional Raman spectroscopy is that high laser powers are required, typically above $20 \mathrm{~mW}$, in combination with long integration times on

\footnotetext{
${ }^{4}$ From this point forward intensity is used although a photon flux is measured.
} 
the order of 10's of seconds and greater, which can cause sample photodecomposition and degradation. To overcome these problems, there are methods that can increase the scattering cross sections, thus the Raman scattered intensities, such as resonance Raman scattering [14,15], coherent anti-Stokes Raman scattering (CARS) [16], and surface-enhanced Raman scattering (SERS) [17]. In this work, SERS is used to improve the limit of detection of surface Raman spectroscopy, which is described in more detail in subsequent chapters.

\subsection{Surface-enhanced Raman spectroscopy}

\subsubsection{Surface-enhanced Raman scattering}

SERS was first experimentally observed by Fleischmann et al. in 1974 when studying pyridine adsorbed on electrochemically roughened silver electrodes; however, the increase of Raman signals were attributed to an increased surface area [18]. Three years later, in 1977, Jeanmaire and Van Duyne [19], and independently Albrecht and Creighton [20], observed similar phenomena and attributed the increased Raman intensity to a new phenomena. In 1978, Moskovits attributed the increased scattering cross section to the excitation of surface plasmons on the rough silver surfaces [21]. Two mechanisms have been proposed to explain the total SERS enhancement: an electromagnetic field enhancement due to surface plasmon resonances [17], and a charge-transfer chemical enhancement [22], both from analytes physisorbed or chemisorbed on the surface of a metallic nanostructure. Many organic and inorganic ions tend to physisorb on metal surfaces.

Measured SERS spectra often differ from conventional Raman scattering spectra due to the interaction of the molecule and the metal surface. Figure 2.3 illustrates differences between a SERS spectrum of a benzenethiol (BT) self-assembled monolayer (SAM) on a gold metal nanowire array surface, described in Section 2.3.3, and a conventional Raman scattering spectrum from a neat BT solution. Most of the vibration bands present in the conventional Raman scattering spectrum are present in the SERS spectrum. Note the difference in the measurement conditions. The center frequencies of SERS vibration bands contain some differences. The largest peak intensity at $\Delta v \approx 1072 \mathrm{~cm}^{-1}$ represents a ring-breathing mode in the SERS spectrum. However, in the conventional Raman spectrum the peak intensity of the ring-breathing mode occurs at $\Delta v \approx 1000 \mathrm{~cm}^{-1}$; the frequency shift is attributed to chemisorption of BT to the gold surface. The intensity peak at $917 \mathrm{~cm}^{-1}$ of the conventional Raman spectrum is attributed to the S-H bending vibration, which disappears in the SERS spectrum, thus indicating S-Au chemisorption, as expected. 


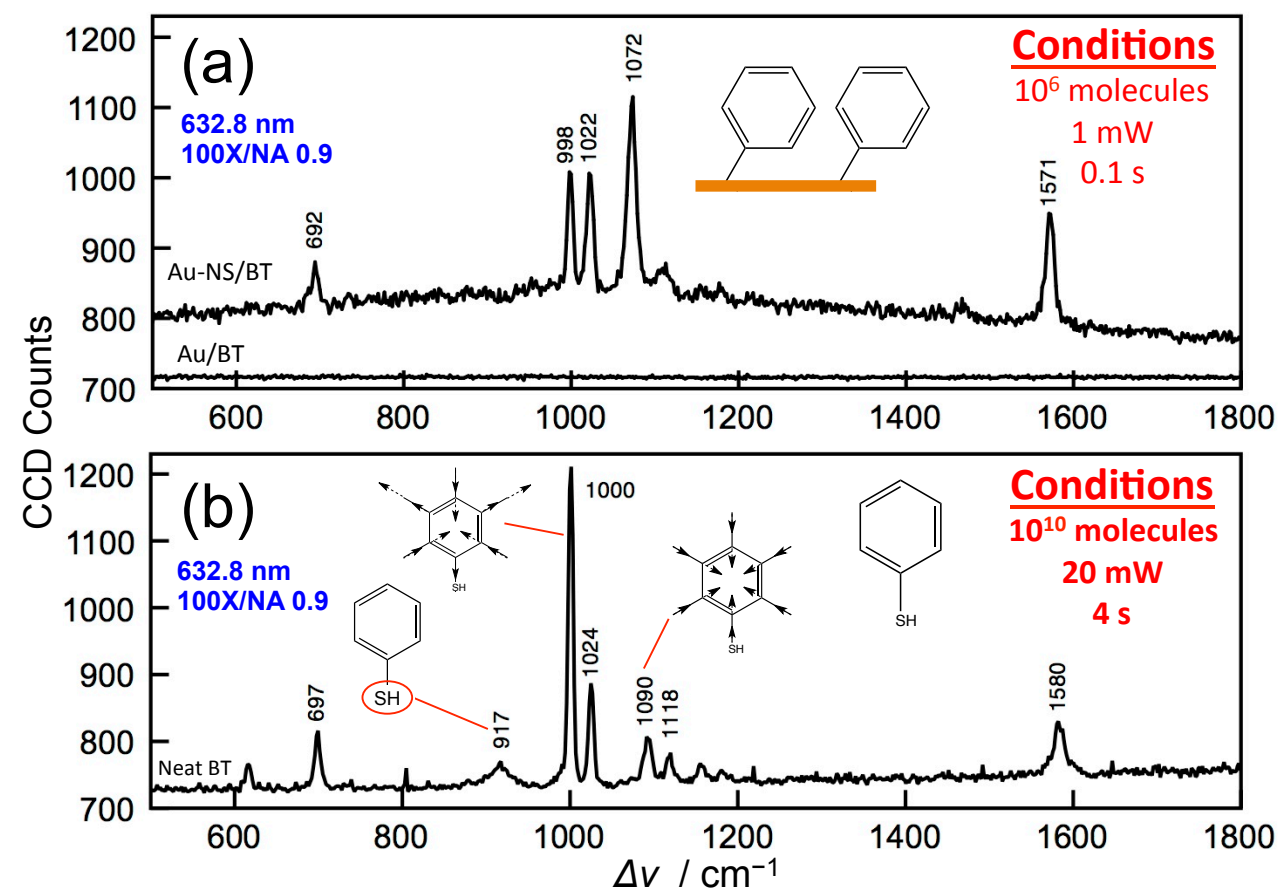

Figure 2.3. Comparison of SERS and conventional Raman scattering spectra from benzenethiol, where $\Delta v$ is the Raman shift. (a) SERS from BT SAM on gold nanowire array surface, (b) conventional Raman scattering from neat BT solution.

In general, many of the vibration bands are shifted in frequency in the SERS spectrum due to $\mathrm{S}-\mathrm{Au}$ chemisorption. Note that the BT SAM on the planar gold surface does not produce any detectable Raman bands. In addition, since the Raman effect is very weak, a large number of BT molecules, on the order of $10^{10}$, are contained in the confocal volume of the measurement system for the neat solution. A relatively high laser power and long integration time are required to produce measurable vibration intensities. These requirements are drastically reduced for the SERS measurement with about $10^{6}$ BT molecules in the collection area, $1 \mathrm{~mW}$ laser power, and $100 \mathrm{~ms}$ integration time; reductions of $10,000 \times, 10 \times$, and $40 \times$, respectively. Furthermore, vibrational modes in close proximity to the gold surface are preferably enhanced, which can provide better differentiation of species compared to the conventional Raman spectra. Interestingly, the SERS spectrum contains a broad background continuum, which is due to plasmon-enhanced photoluminescence from the gold surface; the topic of Chapter 5 .

\subsubsection{SERS enhancement}

SERS results from molecules positioned in the enhanced electric field near the interface between the metal nanostructure and the surrounding dielectric medium. The measured SERS intensity [unit: photons $s^{-1}$ ] of a particular vibration band is estimated as

$$
\Phi_{S}=\Phi_{0}\left(d \sigma_{s} / d \Omega\right) \kappa \sum_{j=1}^{N} G_{j}^{E M} G_{j}^{C H}
$$

where $j$ represents each molecule in the measurement collection volume, i.e. the spot size of 
the optical collection system for a monolayer on the surface, $G^{\mathrm{EM}}$ is the electromagnetic enhancement factor, and $G^{\mathrm{CH}}$ is the chemical enhancement [17]. The electromagnetic enhancement can be estimated as

$$
G_{j}^{E M}(\boldsymbol{r}, \omega)=M_{j}\left(\boldsymbol{r}, v_{0}\right) \cdot M_{j}\left(\boldsymbol{r}, v_{0}-v_{s}\right),
$$

where $M_{j}$ is related to the electromagnetic enhancement factor from the surface plasmon resonance of the metal nanostructure at location $j$, and will be described in a later section. It is commonly accepted that the electromagnetic enhancement dominates, and therefore, the measured intensity can be simplified to

$$
\Phi_{S} \approx \Phi_{0}\left(d \sigma_{s} / d \Omega\right) \kappa N\left\langle G^{E M}\right\rangle
$$

where $\left\langle G^{\mathrm{EM}}\right\rangle$ is an ensemble average enhancement factor from $N$ molecules in the collection volume at each measurement location; a similar effect was reported for fluorescence spectroscopy, which can significantly affect the measured Raman band intensities [23]. The number of molecules in a monolayer $N$ is related to the $D d z$ term in Eq. (2.6). Based on Eq. (2.9), the SERS intensity generated in collection area $A_{\mathrm{s}}$ can be estimated by

$$
\Phi_{S} \approx\left(\lambda_{0} / h c\right) I_{0} n_{d}\left(d \sigma_{s} / d \Omega\right) \kappa N A_{s}^{-1}\left\langle G^{E M}\right\rangle,
$$

where $n_{\mathrm{d}}$ is the refractive index of the medium surrounding the metal nanostructure, $N$ is the number of molecules in the collection area $A_{\mathrm{s}}$, and $\kappa$ is a collection efficiency parameter. For example, the number of chemisorbed BT molecules probed in the collection area of the microscope objective on a gold nanowire array surface is estimated as $N \approx 3 \times 10^{6}$ molecules ${ }^{5}$ (Fig. 2.3(a)). The SERS spectrum shown in Fig. 2.3(a) was measured in air using a He-Ne laser with $1 \mathrm{~mW}$ power and $100 \mathrm{~ms}$ integration time on the BT SAM surface. Assuming a differential scattering cross section of a particular vibration mode of benzenethiol is $\mathrm{d} \sigma_{s} / \mathrm{d} \Omega \approx 10^{-29} \mathrm{~cm}^{2}$ molecule ${ }^{-1} \mathrm{~s}^{-1}$, and an enhancement factor of $\left\langle G^{\mathrm{EM}}\right\rangle=10^{7}$, the total scattered photon flux, estimated from Eq. (2.10), is $\Phi_{\mathrm{S}} \approx 2 \kappa \times 10^{8}$ photons $\mathrm{s}^{-1}$. Furthermore, assuming $1 \%$ of the total of generated photons make it to the detector [4] then about $2 \times 10^{5}$ photons would be detected in $100 \mathrm{~ms}$, which is the same order of magnitude as the photon flux generated by the $1068 \mathrm{~cm}^{-1}$ band of BT on a gold nanowire array surface [25]. Although the Raman intensity can be roughly estimated in this way, in practice it is more useful to construct a calibration curve of the observed Raman intensity as a function sample concentration.

\footnotetext{
${ }^{5} N=A_{\mathrm{s}} D_{\mathrm{BT}} \approx 3 \times 10^{6}$ molecules, where $A_{\mathrm{s}}=1 \times 10^{-12} \mathrm{~m}^{2}$ is from the SERS surface area, and $D_{\mathrm{BT}} \approx 3 \times 10^{18}$ molecules $\mathrm{m}^{-}$ 2 is the surface density of BT molecules chemisorbed on a $\mathrm{Au}(111)$ surface [24]. A gold nanowire array surface with pitch $\Lambda_{\mathrm{g}}=100 \mathrm{~nm}$, nanogap spacing $d_{\mathrm{g}}=20 \mathrm{~nm}$, and a half-ellipse cross section $\left(e_{\mathrm{x}}=40 \mathrm{~nm}\right.$ and $\left.e_{\mathrm{z}}=40 \mathrm{~nm}\right)$ is used. The spot size diameter of the microscope objective is on the order of $1 \mu \mathrm{m}$, which includes 10 nanogaps.
} 


\subsubsection{Surface selection rules}

The surface selection rules of a vibration system, which is composed of an adsorbed molecule on a metal surface, define the vibrations that have zero composite transition dipoles parallel to the surface and the transition dipoles of vibrations perpendicular to the surface that are supported in the molecule-metal system [26]. Consider an infinite cylinder, as shown in Fig. 2.4(a), with radius $a$, and length $L$, such that $L>>a$, which is referred to as a single nanowire. Since the wavelength of the incident electromagnetic radiation is $\lambda>>a$, uniform polarization of the nanowire cross section is assumed using the electrostatic approximation (Fig. 2.4(a)) [27]. The electric field near the surface $(r=a)$ due to the polarization of the nanowire is $\boldsymbol{E}(\boldsymbol{r}, v)=\left(2 \varepsilon_{2} \varepsilon_{0}\right)^{-1}[(\boldsymbol{P} \cdot \hat{r}) \hat{r}-\boldsymbol{P}]$, where $\varepsilon_{2}$ is the permittivity of the surrounding dielectric medium, $\varepsilon_{0}$ is the vacuum permittivity, $\boldsymbol{P}$ is the polarization of the nanowire, and $\hat{r}$ is the radial unit vector of the cylindrical coordinates ${ }^{6}$.
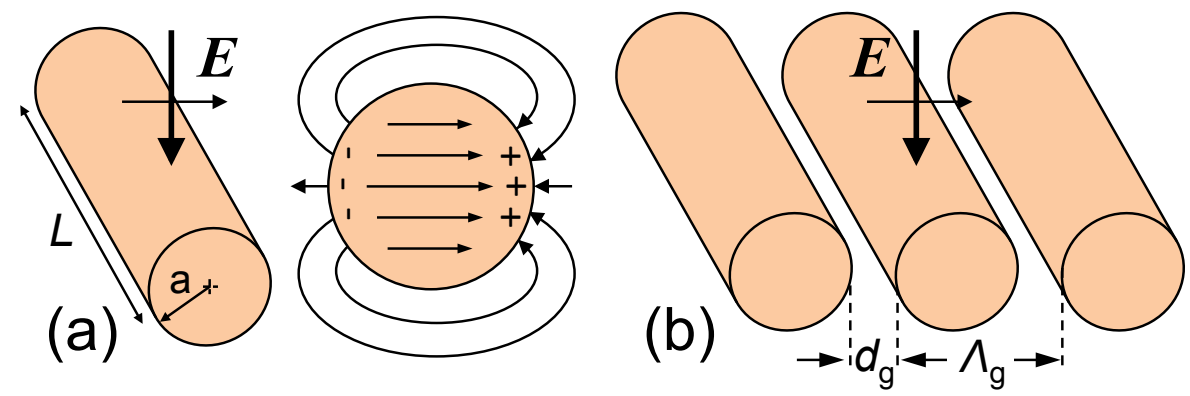

Figure 2.4. (a) Dipolar localized surface plasmon resonance response of infinite cylinder with radius $a$ and length $L$, (b) Closely spaced cylindrical nanoparticles with separation $d_{\mathrm{g}}$ and pitch $\Lambda_{\mathrm{g}}$ and excited polarized electromagnetic radiation along the inter-particle axis.

The polarization of the nanowire is $\boldsymbol{P}=2 \varepsilon_{2} \varepsilon_{0} g(v) \boldsymbol{E}$, with the electromagnetic enhancement factor $g(v)=\left[\varepsilon_{1}(v)-\varepsilon_{2}\right]\left[\varepsilon_{1}(v)+\varepsilon_{2}\right]^{-1}$, the frequency dependent dielectric function of the metal $\varepsilon_{1}(v)$, and the electric field of the incident radiation field $\boldsymbol{E}$. Therefore, the electric field in the near zone generated by the polarized nanowire is $\boldsymbol{E}_{s}=-g(v) E \hat{x}+2 g(v) E \cos \phi \hat{r}$, where $\hat{x}$ is the unit vector in the $x$-direction. The total electric field at the surface of the nanowire $(r=a)$ is $\boldsymbol{E}_{t}=(1-g(v)) E \hat{x}+2 g(v) E \cos \phi \hat{r}$, which is shown graphically in Fig. 2.5(b). The intensity enhancement factor is defined as $M(\phi, v)=\left|\boldsymbol{E}_{\mathrm{t}}(\boldsymbol{r}, v)\right|^{2}|\boldsymbol{E}|^{-2}$, which can be expressed as $M(\phi, v)=M_{\mathrm{s}}+M_{\mathrm{p}}$, where $M_{\mathrm{s}}=(1+g(v))^{2} \cos ^{2} \phi$ and $M_{\mathrm{p}}=(1-g(v))^{2} \sin ^{2} \phi$ are perpendicular and parallel to the nanowire surface, respectively. The perpendicular and parallel components of the enhancement factor are important for accessing the surface selection rules. The total enhancement is $M(\phi, v)=(1+g(v))^{2} \cos ^{2} \phi+(1-g(v))^{2} \sin ^{2} \phi$, which indicates that the maximum enhancement perpendicular to the surface occurs when $M(\phi=0, v)=(1+g(v))^{2}$, where the

\footnotetext{
${ }^{6}$ Cylindrical coordinates are defined as $(r, \varphi, z)$ where $x=r \cos \varphi, y=r \sin \varphi$, and $z=z$. 
parallel component of the electric field is zero, and the maximum enhancement parallel to the surface occurs when $M(\phi=\pi / 2, v)=(1-g(v))^{2}$, where the perpendicular component of the electric field is ideally zero. The orientation of molecules adsorbed uniformly over the surface leads to an average enhancement of the nanowire surface where the perpendicular and parallel components provide approximately the same contribution, in contrast to a spherical nanoparticle. The corresponding Raman scattering enhancement is $G=M(v) \cdot M\left(v_{\mathrm{s}}\right)$, where $v$ and $v_{\mathrm{s}}$ are the frequencies of the incident and scattered radiation, respectively.
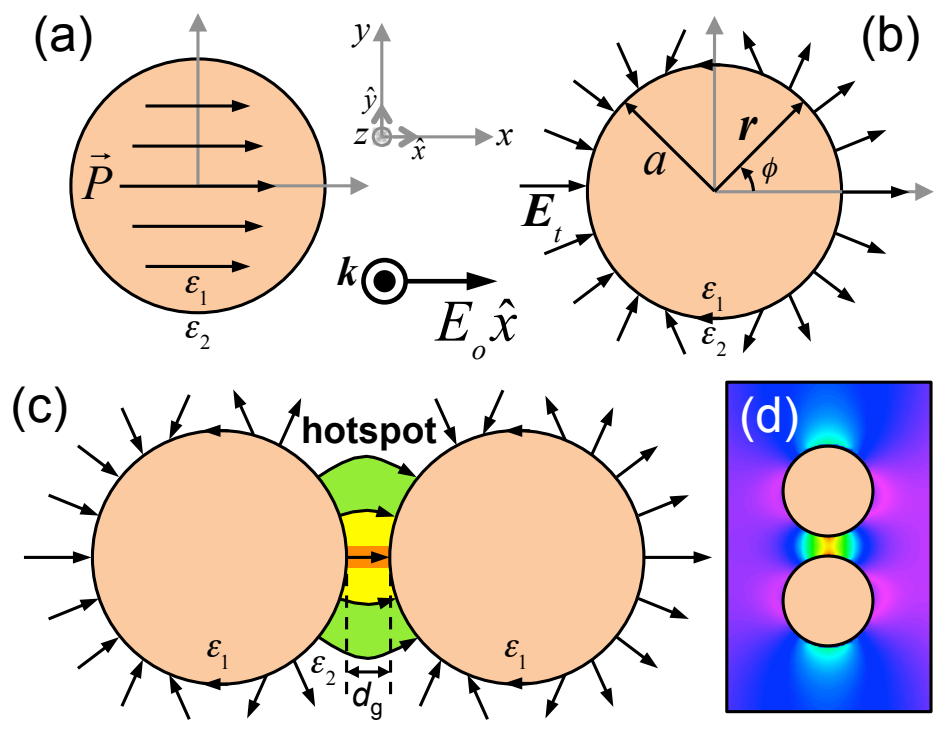

Figure 2.5. Polarization and electric field characteristics of cylindrical nanowire using the electrostatic approximation. (a) Uniform polarization of a single nanowire, (b) Electric near-field profile of a single nanowire, (c) Electric near-field profile and hotspot region of a nanowire dimer, (d) Simulated electric near-field profile of a nanowire dimer with $d_{\mathrm{g}}=10 \mathrm{~nm}$.

The maximum Raman enhancement of a single nanowire is $G_{\max } \propto(1+g(v))^{2}\left(1+g\left(v_{\mathrm{s}}\right)\right)^{2}$, which occurs at $x=a$ and $y=0$ along the $z$-axis of the nanowire, which is about $16 \times$ smaller than the maximum enhancement produced by a spherical nanoparticle [28]. When two metal nanowires are positioned in close proximity to each other (Fig. 2.4(b)), the electric field in the near zone generated by one nanowire interacts with the electric field in the near zone of the adjacent nanowire. Therefore, the total electric field acting on the nanowire is a combination of the incident electric field and the electric field from the adjacent nanowire; the nanowire dimer excitation is $\boldsymbol{E}_{\mathrm{t}}+\boldsymbol{E}_{\mathrm{s}}$. This near-field interaction results in a red-shifted coupled plasmon resonance and an increased electric field magnitude in the nanogap region between the adjacent nanowires, compared to the near region of a single nanowire. The electric field in the near zone is drastically modified when the nanogap dimensions are reduced to the nanometer scale. Nanogaps with $d_{\mathrm{g}} \leq 20 \mathrm{~nm}$ are called hotspots due the large electric field enhancement generation induced by the coupled plasmon resonance, as shown in the illustration of a nanowire-dimer pair in Fig. 2.5(c). Figure 2.5(d) shows an example of a simulated electric 
near-field profile showing the hotspot region located in the junction between parallel nanowires, where the red region of the hotspot can generate $M \sim 10^{4}$ [29].

The coupled plasmon resonances have important consequences for surface-enhanced Raman scattering of molecules adsorbed on the metal nanowire array surfaces. Since the electromagnetic field enhancement in the hotspot region is orders of magnitude larger than an enhancement generated by an isolated nanostructure, the measured signal is comprised largely from the scattering of molecules located in the hotspot region (Fig. 2.5(c)), where the electric field perpendicular to the surface is highly amplified.
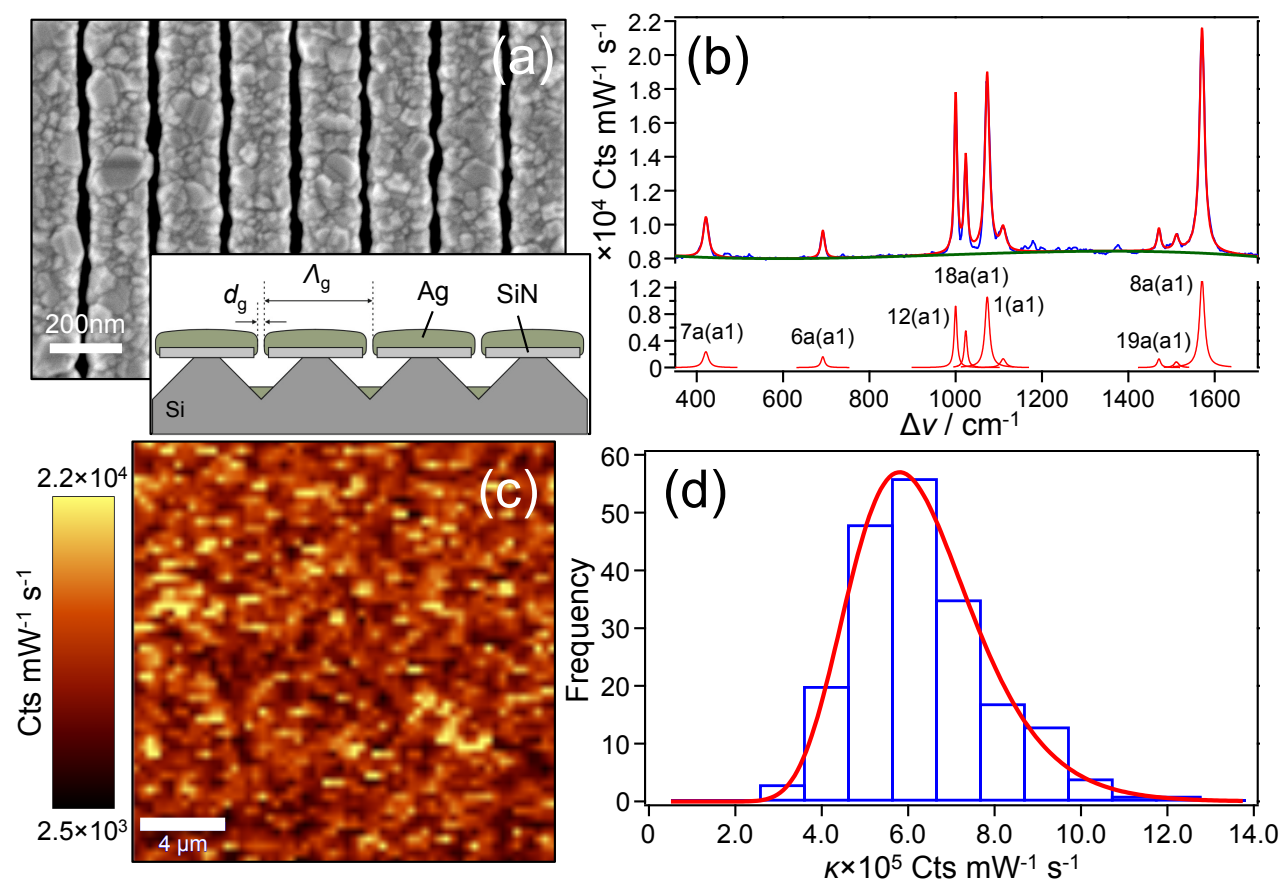

Figure 2.6. Silver nanowire array surface. (a) SEM image of typical surface. Inset: cartoon of the surface cross section (inset: excitation laser polarization), (b) Typical BT-Ag SERS spectrum (the blue trace is the experimental data, the red trace is the modeled data, and the green trace corresponds to the modeled background). The bottom part shows the bands obtained from the modeling procedure. Inset: Schematic BT SAM, (c) Raman image of the integrated intensities $\kappa_{\mathrm{i}}$ of the $8 \mathrm{a}\left(\mathrm{a}_{1}\right), v(\mathrm{C}-\mathrm{C})$ vibration over the imaged region, (d) Histogram of the distribution of $\kappa_{i}(n=200)$ of the $8 \mathrm{a}\left(\mathrm{a}_{1}\right), v(\mathrm{C}-\mathrm{C})$ vibration mode over the imaged region.

For molecules located in the nanogap region, a vibration with symmetry perpendicular to the surface will be enhanced to a greater extent compared to a vibration with symmetry parallel to the surface. This can be assessed using a BT SAM on a silver nanowire array surface, since the BT-Ag SAM surface density and orientation are well established [6,30]. BT belongs to the $C_{2 \mathrm{v}}$ point group and is composed of $\mathrm{a}_{1}, \mathrm{a}_{2}, \mathrm{~b}_{1}$ and $\mathrm{b}_{2}$ vibration mode symmetries that are distributed as: $11 a_{1}+10 b_{2}+3 a_{2}+6 b_{1}$ [31]. The $a_{1}$ modes are composed of a linear combination of $\alpha_{\mathrm{xx}}$ and $\alpha_{\mathrm{yy}}$, oriented parallel to the surface, and $\alpha_{\mathrm{zz}}$, which is oriented perpendicular to the surface, where $\alpha_{\mathrm{ij}}$ are components of the polarizability tensor $\alpha$ with excitation polarization state $i$ and induced molecular polarization state $j$. Note that in this case 
$x$ and $y$ represent orientations parallel to the surface and $z$ represents an orientation perpendicular to the surface. From the Raman spectrum measured from a BT SAM chemisorbed on the silver nanowire array surface, shown in Fig. 2.6(b), twelve vibration modes are resolved compared to fourteen vibration modes observed from the conventional Raman scattering measurements from a neat BT sample [32]. Table 2.4 summarizes the BT measurements where the integrated intensities of each band have been normalized with the intensity of the $12\left(a_{1}\right)$ mode, which is not influenced by the $v(C-S)$ vibration [6].

Table 2.4. SERS of BT SAM on silver nanowire array surface. Mode assignments from [31]. NII is the integrated intensity normalized to the integrated intensity of the $12\left(a_{1}\right)$ vibration mode. The Wilson numbering scheme is used for the vibration modes and symmetry is indicated in parentheses. Labels $\gamma$, $\beta$ and $v$ indicate out-of-plane bending, in-plane bending and stretching modes, respectively.

\begin{tabular}{|c|c|c|c|c|}
\hline Mode assignments & $\begin{array}{c}\text { Neat BT } \\
\Delta v / \mathrm{cm}^{-1}\end{array}$ & $\begin{array}{c}\text { Neat } \\
\text { BT } \\
\text { NII }\end{array}$ & $\begin{array}{c}\text { SERS } \\
\text { BT/Ag } \\
\Delta v / \mathrm{cm}^{-1}\end{array}$ & $\begin{array}{c}\text { SERS } \\
\text { BT/Ag } \\
\text { NII }\end{array}$ \\
\hline $7 \mathrm{a}\left(\mathrm{a}_{1}\right), v(\mathrm{C}-\mathrm{S})+\beta(\mathrm{C}-\mathrm{C}-\mathrm{C})$ & 414 & 0.37 & 421 & 0.49 \\
\hline $16 \mathrm{~b}\left(\mathrm{~b}_{1}\right), \gamma(\mathrm{C}-\mathrm{C}-\mathrm{C})$ & 484 & 0.12 & 473 & 0.04 \\
\hline $6 \mathrm{~b}\left(\mathrm{~b}_{2}\right), \beta(\mathrm{C}-\mathrm{C}-\mathrm{C})+v(\mathrm{C}-\mathrm{S})$ & 619 & 0.11 & - & - \\
\hline $6 \mathrm{a}\left(\mathrm{a}_{1}\right), \beta(\mathrm{C}-\mathrm{C}-\mathrm{C})+v(\mathrm{C}-\mathrm{S})$ & 701 & 0.21 & 691 & 0.22 \\
\hline$\beta(\mathrm{S}-\mathrm{H})$ & 917 & 0.26 & - & - \\
\hline $12\left(\mathrm{a}_{1}\right), \beta(\mathrm{C}-\mathrm{C}-\mathrm{C})$ & 1003 & 1.00 & 1000 & 1.00 \\
\hline $18 \mathrm{a}\left(\mathrm{a}_{1}\right), \beta(\mathrm{C}-\mathrm{H})$ & 1027 & 0.26 & 1023 & 0.59 \\
\hline $1\left(\mathrm{a}_{1}\right), \beta(\mathrm{C}-\mathrm{C}-\mathrm{C})+v(\mathrm{C}-\mathrm{S})$ & 1095 & 0.27 & 1073 & 1.59 \\
\hline $\begin{array}{c}6 \mathrm{a}\left(\mathrm{a}_{1}\right)+7 \mathrm{a}\left(\mathrm{a}_{1}\right),(\beta(\mathrm{C}-\mathrm{C}-\mathrm{C})+ \\
\mathrm{v}(\mathrm{C}-\mathrm{S}))+(v(\mathrm{C}-\mathrm{S})+\beta(\mathrm{C}-\mathrm{C}-\mathrm{C}))\end{array}$ & 1120 & 0.13 & 1111 & 0.28 \\
\hline $15\left(\mathrm{~b}_{2}\right), \beta(\mathrm{C}-\mathrm{H})$ & 1159 & 0.10 & 1157 & 0.04 \\
\hline $9 \mathrm{a}\left(\mathrm{a}_{1}\right), \beta(\mathrm{C}-\mathrm{H})$ & 1184 & 0.05 & 1178 & 0.09 \\
\hline $19 \mathrm{a}\left(\mathrm{a}_{1}\right), v(\mathrm{C}-\mathrm{C})$ & - & - & 1471 & 0.15 \\
\hline $8 \mathrm{a}\left(\mathrm{a}_{1}\right), v(\mathrm{C}-\mathrm{C})$ & 1586 & 0.44 & 1571 & 1.82 \\
\hline$v(\mathrm{~S}-\mathrm{H})$ & 2568 & 0.89 & - & - \\
\hline $2\left(\mathrm{a}_{1}\right), v(\mathrm{C}-\mathrm{H})$ & 3058 & 0.98 & 3047 & 0.36 \\
\hline
\end{tabular}

Nine of the resolved modes have $a_{1}$ symmetry, which results from the large enhancement perpendicular to the surface in the hotspot region. The $a_{2}$ symmetry modes are weak in benzene and its derivatives, [31] and are not observed in either the conventional Raman or the SERS measurements. Additionally, the $\mathrm{a}_{2}$ symmetry vibrations are least enhanced for BT oriented perpendicular to the surface [28]. The $b_{1}$ modes are composed of the $\alpha_{x z}$ component and $b_{2}$ modes contain the $\alpha_{y z}$ component. Very weak $16 b\left(b_{1}\right)$ and $15\left(b_{2}\right)$ modes have been resolved in the SERS measurements. Since the $b_{1}$ and $b_{2}$ modes result from vibrations parallel to the surface that are excited with a perpendicular field, thus their integrated intensities are very weak and at least an order of magnitude smaller than any of the resolved $a_{1}$ vibration modes. The fact that the integrated intensities of the $16 \mathrm{~b}\left(\mathrm{~b}_{1}\right)$ and $15\left(\mathrm{~b}_{2}\right)$ modes are similar in magnitude indicates that the BT SAM is oriented perpendicular to the surface [28]. Although 
the current results indicate that the BT SAM is oriented perpendicular to the silver surface, the model to justify the surface selection rules is overly simplistic and for definitive assignment a more careful study is required that takes into account charge transfer effects, ambient surface conditions, and the surface roughness of the silver layer. Another outstanding feature of this analysis is that modes $1\left(a_{1}\right)$ and $8 a\left(a_{1}\right)$ from the SERS measurements have much larger normalized ratios compared to the conventional Raman measurements. The increase of the $1\left(a_{1}\right)$ mode is attributed to the coupling of the $v(C-S)$ stretch with the $\beta(C-C-C)$ ring stretch, which is in close proximity to the surface. However, the integrated intensity of the $8 a\left(a_{1}\right)$ mode is the largest of all the observed bands, which is in contrast to the conventional Raman measurements and from BT-Au SERS measurements [25].

\subsection{SERS as a "standard" analytical technique}

Practical quantitative analytical techniques require measurements to be reproducible with established standards for calibration, as well as standard sample preparation methods. Nearly 40 years since the discovery of SERS, surface-enhanced Raman spectroscopy continues to evolve as an important analytical techniques due to its improved detection limit compared to conventional Raman spectroscopy, and its ability to identify chemical species and provide structural information of various materials in material science, biochemistry, catalysis, and electrochemistry [33].

Surface-enhanced Raman spectroscopy is a suitable technique to assess the orientation of molecules on the surface. Cunningham, et al. [34] showed that pyridine monolayers on silver surfaces tends to lie with the ring plane parallel to the surface, but tend to stack with the plane perpendicular to the surface. As a result, the SERS intensities of in-plane carbon vibrations increase at higher concentrations because they have larger polarizability normal to the surface. Surface-enhanced Raman spectroscopy has also been reported for use in conjunction with electrochemistry, which combines Raman spectroscopy and electrochemistry to study electrochemically active molecules. Based on changes in vibrational frequencies, relative peak intensities and background, one can monitor changes in oxidation states, structural changes or redox properties $[35,36]$. Single molecule surface-enhanced Raman spectroscopy is another important application area, where single molecule spectra are usually based on surface-enhanced resonance Raman scattering, and offers some advantages, compared to single molecule fluorescence measurements, such as decreased sample bleaching and richer molecular structural information. The bi-analyte method, introduced by Le Ru, et al. [37], is based on a pair of molecularly similar molecules, for example, isotopologues, or nondeuterated/deuterated pairs, which provide more quantitative counting [38]. Since most 
samples are composed of many components, separation techniques such as capillary electrophoresis or liquid chromatography can be used in combination with surface-enhanced Raman spectroscopy to isolate different analytes before identifying the samples $[39,40]$. Since surface-enhanced Raman spectroscopy can provide ultrasensitive detection it is appealing for integration with lab-on-a chip, or microfluidic, systems for biomedical and life science applications $[41,42]$.

A key step towards the success of surface-enhanced Raman spectroscopy as a standard analytical technique relies on the SERS-active substrate. The first SERS-active substrate was a roughened silver electrode [18]; however, metallic nanoparticles of various shapes and sizes prepared by chemical synthesis methods are commonly used currently. The main methods used to prepare metal nanoparticles include chemical reduction, laser ablation and photoreduction [43-51]. Metal nanoparticle colloids prepared by chemical methods are problematic because of the influence of residual ions on the SERS signal. To overcome this problem, laser ablation or photoreduction is used to produce silver, gold, copper, and platinum colloids [52-56]. Since molecules residing in close proximity to small nanogap regions created by two metal nanoparticles will give extremely strong Raman scattering, as previously described, many groups have attempted to form aggregates of two or multiple nanoparticles. A common approach for aggregation is by adding substances to the colloid solutions, such as inorganic salts $\mathrm{KCl}$ and $\mathrm{NaCl}$, surfactants, and organic amines [57,58]. Nie and co-workers reported that they achieved a surface-enhanced resonance Raman scattering enhancement factor as large as $10^{14}$ by adding chloride ions to a silver colloid solution and detected single rhodamine-6G molecules $[59,60]$. In retrospect, the single molecule spectra likely originated from electromagnetic hotspots located between adjacent nanoparticles, which was not discussed in the original report. Other groups have reported extremely large surface-enhanced resonance Raman scattering enhancement factors on gold nanoparticle aggregates [61] and 1$2 \mathrm{~nm}$ gap dimers [62-64]. Using nanoparticles as a SERS substrate offers advantages, such as low cost and ease of manipulation; the use of colloidal solutions permits the acquisition of an average spectrum due to the Brownian motion that governs colloidal dispersions [65]. There are a large number of reports of realizing metal nanoparticles, dimers, and aggregates into SERS-active substrates. However, non-uniformity and irreproducibility remains problematic. Roughened metal surface electrodes, or island metal films, can be simply created by drying the colloidal solution on silicon or glass substrates, physical vapor deposition, and electrochemical processing; however, the enhancement factor of these substrates is highly non-uniform. 
Nowadays, with the use of nanofabrication methods, lithographically patterned SERS substrates remain one of the most promising methods to reproducibly produce large and high density arrays of hotspots due to their commercial availability and wide spread expertise. In general, SERS-active solid support substrates offer several benefits: firstly, the surfaces are typically stable and can be stored for long period of time between fabrication and use; secondly, it is easier to make measurements when the excitation beam is properly focused; and lastly, a SERS surface can be interfaced to external electrochemical instrumentation. Different nanolithography techniques have been used to realize SERS-active surfaces, such as focused ion beam milling, nanoimprinting, and electron-beam lithography. These advanced techniques allow the generation of nanostructured metal surfaces with various shapes, sizes and gap separations [66,67]. Many groups have reported highly ordered arrays, including nanoholes [68], nanoslits [69], and in particular commercially available inverted pyramidal pits (Klarite 302, Renishaw Diagnostics, Ltd) [70]. Subwavelength metallic nanogratings are an important class of SERS-active substrates, as described in this thesis, and have been reported for over 30 years [25,32,71-75].

Despite the impressive progress made in surface-enhanced Raman spectroscopy over the past four decades, there remain areas that require improvement. First, there remains a need for reliable and reproducible SERS substrates, as mentioned above. Second, a much deeper understanding of the electromagnetic enhancement and chemical enhancement mechanisms are required in order to further improve the technique. Third, although significant progress has been made in developing reliable SERS-active substrates, relatively little has been done to advance practical applications for surface-enhanced Raman spectroscopy. As more application areas for surface-enhanced Raman spectroscopy emerge, SERS/SERRS spectral libraries will be essential to aid analytical scientists in quantitatively sample identification.

\section{References}

[1] D.A. Long, The Raman Effect. John Wiley \& Sons, 2002, p. 661.

[2] R. Singh, "C.V. Raman and the discovery of the Raman effect," Phys. Perspect. 4, 399420, 2002.

[3] G. Turrell and J. Corset, Raman Microscopy. Elsevier, 1996.

[4] R.L. McCreery, Raman Spectroscopy for Chemical Analysis. John Wiley \& Sons. 2000.

[5] M. Morris and D.J. Wallan, "Resonance Raman spectroscopy," Anal. Chem. 51, 182A192A, 1979.

[6] K.T. Carron and L.G. Hurley, "Axial and azimuthal angle determination with surfaceenhanced Raman spectroscopy: Thiophenol on copper, silver, and gold metal surfaces," $J$. Phys. Chem. 95, 9979-9984, 1991.

[7] T.G. Spiro and T.C. Strekas, "Resonance Raman spectra of heme proteins. Effects of oxidation and spin state," J. Am. Chem. Soc. 570, 338-345, 1974. 
[8] L.S. Alexander and H.M. Goff, "Chemicals, cancer, and cytochrome P-450," J. Chem. Educ. 59, 179, 1982.

[9] J.R. Ferraro, K. Nakamoto, and C.W. Brown, Introductory Raman Spectroscopy. Elsevier, 2003, pp. 406-421.

[10] H. Haken and H.C. Wolf, Molecular Physics and Elements of Quantum Chemistry. Springer, 1995.

[11] J. Schmidt and A. Penzkofer, "Absorption cross-section and density measurement of dye vapors," Chem. Phys. 117, 265-273, 1987.

[12] J.G. Skinner and W.G. Nilsen, "Absolute Raman scattering cross-section measurement of the $992 \mathrm{~cm}^{-1}$ line of benzene," J. Opt. Soc. Am. 58, 1968.

[13] D. Wu, M. Hayashi, S. Lin, and Z. Tian, "Theoretical differential Raman scattering cross-sections of totally-symmetric vibrational modes of free pyridine and pyridine-metal cluster complexes," Spectrochim. Acta A, 60, 137-146, 2004.

[14] S.A. Asher, "UV resonance Raman spectroscopy for analytical, physical and biophysical chemistry, part 1," Anal. Chem. 65, 59A-66A, 1993.

[15] S.A. Asher, "UV resonance Raman spectroscopy Part 2," Anal. Chem. 65, 201A-210A, 1993.

[16] P.R. Régnier and J.P.-E. Taran, "On the possibility of measuring gas concentrations by stimulated anti-Stokes scattering," Appl. Phys. Lett. 23, 240-242, 1973.

[17] M. Moskovits, "Surface-enhanced spectroscopy," Rev. Mod. Phys. 57, 783-826, 1985.

[18] M. Fleischmann, P.J. Hendra, and A.J. McQuillan, "Raman spectra of pyridine adsorbed at a silver electrode," Chem. Phys. Lett. 26, 163-166, 1974.

[19] D.L. Jeanmaire and R.P. Van Duyne, "Surface Raman spectroelectro- chemistry part I. Heterocyclic, aromatic, and aliphatic amines adsorbed on the anodized silver electrode," J. Electroanal. Chem. 84, 1-20, 1977.

[20] M.G. Albrecht and J.A. Creighton, "Anomalously intense Raman spectra of pyridine at a silver electrode," J. Am. Chem. Soc. 99, 5215-5217, 1977.

[21] M. Moskovits, "Surface roughness and the enhanced intensity of Raman scattering by molecules adsorbed on metals," J. Chem. Phys. 69, 4159, 1978.

[22] A. Otto, "The 'chemical' (electronic) contribution to surface-enhanced Raman scattering," J. Raman Spectrosc. 36, 497-509, 2005.

[23] W.E. Moerner, "Examining nanoenvironments in solids on the scale of a single, isolated impurity molecule," Science 265, 46-53, 1994.

[24] L.-J. Wan, M. Terashima, H. Noda, and M. Osawa, "Molecular orientation and ordered structure of benzenethiol adsorbed on gold(111)," J. Phys. Chem. B 104, 3563-3569, 2000.

[25] L. Le Thi Ngoc, M. Jin, J. Wiedemair, A. van den Berg, and E.T. Carlen, "Large area metal nanowire arrays with tunable sub-20 nm nanogaps," ACS Nano 7, 5223-5234, 2013.

[26] M. Moskovits, “Surface selection rules,” J. Chem. Phys. 77, 4408, 1982.

[27] J.D. Jackson, Classical Electrodynamics. John Wiley \& Sons, 1975.

[28] J.A. Creighton, "Surface Raman electromagnetic enhancement factors for molecules at the surface of small isolated metal spheres," Sur. Sci. 124, 209-219, 1983.

[29] F.J. Garcia-Vidal and J.B. Pendry, "Collective theory for surface enhanced Raman scattering," Phy. Rev. Lett. 77, 1163-1166, 1996.

[30] J.Y. Gui, D.A. Stern, D.G. Frank, F. Lu, D.C. Zapien, and A.T. Hubbard, "Adsorption and surface structural chemistry of thiophenol, benzyl mercaptan, and alkyl mercaptans. Comparative studies at $\operatorname{Ag}(111)$ and $\operatorname{Pt}(111)$ electrodes by means of Auger spectroscopy, electron energy loss spectroscopy, low-energy electron diffraction and electrochemistry," Langmuir 7, 955-963, 1991. 
[31] G. Varsanyi, Vibrational Spectra of Benzene Derivates. Academic Press, 1969.

[32] J. Wiedemair, L. Le Thi Ngoc, A. van den Berg, and E.T. Carlen, "Surface-enhanced Raman spectroscopy of self-assembled monolayer conformation and spatial uniformity on silver surfaces," J. Phys. Chem. C 118, 11857-11868, 2014.

[33] B. Sharma, R.R. Frontiera, A.-I. Henry, E. Ringe, and R.P. Van Duyne, "SERS: Materials, applications, and the future," Mater. Today 15, 16-25, 2012.

[34] D. Cunningham, R.E. Littleford, W.E. Smith, P.J. Lundahl, I. Khan, D. W. McComb, D. Graham, and N. Laforest, "Practical control of SERRS enhancement," Faraday Discuss. 132, 135, 2006.

[35] R.P. Van Duyne and J.P. Haushalter, "Resonance Raman spectroelectrochemistry of semiconductor electrodes," J. Phys. Chem. 88, 2446-2451, 1984.

[36] W.F. Paxton, S.L. Kleinman, A.N. Basuray, J.F. Stoddart, and R.P. Van Duyne, "Surface-enhanced Raman spectroelectrochemistry of TTF-modified self-assembled monolayers," J. Phys. Chem. Lett., 2, 1145-1149, 2011.

[37] E.C. Le Ru, M. Meyer, and P.G. Etchegoin, "Proof of single-molecule sensitivity in surface enhanced Raman scattering (SERS) by means of a two-analyte technique," $J$. Phys. Chem. B 110, 1944-1948, 2006.

[38] J.A. Dieringer, R.B. Lettan II, K.A. Scheidt, and R.P. Van Duyne, “A frequency domain existence proof of single-molecule surface-enhanced Raman spectroscopy," J. Am. Chem Soc. 129, 16249-16256, 2007.

[39] G.L. DeVault and M.J. Sepaniak, "Spatially focused deposition of capillary electrophoresis effluent onto surface-enhanced Raman-active substrates for off-column spectroscopy," Electrophoresis, 22, 2303-2311, 2001.

[40] S.A. Soper, K.L. Ratzlaff, and T. Kuwana, "Surface-enhanced resonance Raman spectroscopy of liquid chromatographic analytes on thin-layer chromatographic plates," Anal. Chem. 62, 1438-1444, 1990.

[41] J. Popp, A. März, K. Ackermann, P. Rösch, and T. Henkel, "SERS measurements in microfluidic devices: A promising way for online-monitoring of lowest agent concentrations," Proc. of SPIE, vol. 7593, pp. 759303-759309, 2010.

[42] W. Kiefer, Surface Enhanced Raman Spectroscopy Analytical, Biophysical and Life Science Applications. Wiley-VCH Verlag, 2011.

[43] Y. Wang, D. Li, P. Li, W. Wang, W. Ren, S. Dong, and E. Wang, "Surface enhanced Raman scattering of Brilliant-Green on Ag nanoparticles and applications in living cells as optical probes," J. Phys. Chem. C 111, 16833-16839, 2007.

[44] M.-F. Huang, Y.-C. Kuo, C.-C. Huang, and H.-T. Chang, "Separation of long doublestranded DNA by nanoparticle-filled capillary electrophoresis," Anal. Chem. 76, 192196, 2004.

[45] P.C. Lee and D. Meisel, "Adsorption and Surface-enhanced Raman of dyes on silver and gold sols," J. Phys. Chem. 86, 3391-3395, 1982.

[46] G.C. Weaver and K. Norrod, "Surface enhanced Raman spectroscopy: A novel physical chemistry experiment for the undergraduate laboratory," J. Chem. Edu. 75, 621, 1998.

[47] U. Nickel, K. Mansyreff, and S. Schneider, "Production of monodisperse silver colloids by reduction with hydrazine: the effect of chloride and aggregation on SER(R)S signal intensity," J. Raman Spectrosc. 35, 101-110, 2004.

[48] M.V. Canamares, J.V. Garcia-Ramos, J.D. Gomez-Varga, C. Domingo, and S. SanchezCortes, "Comparative study of the morphology, aggregation, adherence to glass, and surface-enhanced Raman scattering activity of silver nanoparticles prepared by chemical reduction of Ag+ using citrate and hydroxylamine," Langmuir, 21, 8546-8553, 2005. 
[49] N. Leopold and B. Lendl, "A new method for fast preparation of highly surface-enhanced Raman scattering (SERS) active silver colloids at room temperature by reduction of silver nitrate with hydroxylamine hydrochloride," J. Phys. Chem. B 107, 5723-5727, 2003.

[50] U. Nickel, A.Z. Castell, K. Pöppl, and S. Schneider, "A silver colloid produced by reduction with hydrazine as support for highly sensitive surface-enhanced Raman spectroscopy," Langmuir 16, 9087-9091, 2000.

[51] L. Kvitek, R. Prucek, A. Panacek, R. Novotny, J. Hrbac, and R. Zboril, "The influence of complexing agent concentration on particle size in the process of SERS active silver colloid synthesis," J. Mater. Chem. 15, 1099, 2005.

[52] A.M. Schwartzberg, T.Y. Oshiro, J.Z. Zhang, T. Huser, and C.E. Talley, "Improving nanoprobes using surface-enhanced Raman scattering from 30-nm hollow gold particles," Anal. Chem. 78, 4732-4736, 2006.

[53] S. Besner, A.V. Kabashin, and M. Meunier, "Two-step femtosecond laser ablation-based method for the synthesis of stable and ultra-pure gold nanoparticles in water," Appl. Phys. A-Mater. 88, 269-272, 2007.

[54] M. Prochazka, P. Mojzes, J. Stepanek, B. Vlckova, and P.-Y. Turpin, "Probing applications of laser-ablated Ag colloids in SERS spectroscopy: Improvement of ablation procedure and SERS spectral testing," Anal. Chem. 69, 5103-5108, 1997.

[55] J. Neddersen, G. Chumanov, and T.M. Cotton, "Laser ablation of metals: A new method for preparing SERS active colloids," App. Spectrosc. 47, 1959-1964, 1993.

[56] S. Tan, M. Erol, A. Attygalle, H. Du, and S. Sukhishvili, "Synthesis of positively charged silver nanoparticles via photoreduction of $\mathrm{AgNO}_{3}$ in branched polyethyleneimine/HEPES solutions," Langmuir 24, 9836-9843, 2007.

[57] L. Sun, Y. Song, L. Wang, C. Guo, Y. Sun, Z. Liu, and Z. Li, "Ethanol-induced formation of silver nanoparticle aggregates for highly active SERS substrates and application in DNA detection," J. Phys. Chem. C, 112, 1415-1422, 2008.

[58] S.E.J. Bell and N.M.S. Sirimuthu, "Surface-enhanced Raman spectroscopy (SERS) for sub-micromolar detection of DNA/RNA mononucleotides," J. Am. Chem. Soc. 128, 15580-15581, 2006.

[59] S. Nie and S.R. Emory, "Probing single molecules and single nanoparticles by surfaceenhanced Raman scattering," Science 275, 1102-1106, 1997.

[60] W.E. Doering and S. Nie, "Single-molecule and single-nanoparticle SERS: Examining the roles of surface active sites and chemical enhancement," J. Phys. Chem. B 106, 311$317,2002$.

[61] C. Ruan, W. Wang, and B. Gu, "Single-molecule detection of thionine on aggregated gold nanoparticles by surface enhanced Raman scattering," J. Raman Spectrosc. 38, 568573, 2007.

[62] W. Li, P.H.C. Camargo, X. Lu, and Y. Xia, "Dimers of silver nanospheres: facile synthesis and their use as hot spots for surface-enhanced Raman scattering," Nano Lett. 9, 485-490, 2009.

[63] C.E. Talley, J.B. Jackson, C. Oubre, N.K. Grady, C.W. Hollars, S.M. Lane, T.R. Huser, P. Nordlander, and N.J. Halas, "Surface-enhanced Raman scattering from individual $\mathrm{Au}$ nanoparticles and nanoparticle dimer substrates," Nano Lett. 5, 1569-1574, 2005.

[64] J.M. McMahon, A.-I. Henry, K.L. Wustholz, M.J. Natan, R.G. Freeman, R.P. Van Duyne, and G.C. Schatz, "Gold nanoparticle dimer plasmonics: finite element method calculations of the electromagnetic enhancement to surface-enhanced Raman spectroscopy," Anal. Bioanal. Chem. 394, 1819-1825, 2009.

[65] R.F. Aroca, R.A. Alvarez-Puebla, N. Pieczonka, S. Sanchez-Cortez, and J.V. GarciaRamos, "Surface-enhanced Raman scattering on colloidal nanostructures," Adv. Colloid Interfac. 116, 45-61, 2005. 
[66] S. Kitson, W. Barnes, and J. Sambles, "Full photonic band gap for surface modes in the visible," Phys. Rev. Lett. 77, 2670-2673, 1996.

[67] Z. Li, W.M. Tong, W.F. Stickle, D.L. Neiman, R.S. Williams, L.L. Hunter, and A.A. Talin, "Plasma-induced formation of Ag nanodots for ultra-high-enhancement surfaceenhanced Raman scattering substrates," Langmuir 23, 5135-5138, 2007.

[68] A.G. Brolo, E. Arctander, R. Gordon, B. Leathem, and K.L. Kavanagh, "Nanoholeenhanced Raman scattering," Nano Lett. 4, 2015-2018, 2004.

[69] J.A. Porto, F.J. Garcia-Vidal, and J.B. Pendry, "Transmission resonances on metallic gratings with very narrow slits," Phys. Rev. Lett. 83, 2845-2848, 1999.

[70] N.M.B. Perney, J.J. Baumberg, M.E. Zoorob, M.D.B. Charlton, S. Mahnkopf, and C.M. Netti, "Tuning localized plasmons in nano-structured substrates for surface-enhanced Raman scattering," Opt. Express 14, 824-830, 2006.

[71] J. Tsang, J. Kirtley, and J. Bradley, "Surface-enhanced Raman spectroscopy and surface plasmons," Phys. Rev. Lett. 43, 772-775, 1979.

[72] I. Baltog, N. Primeau, R. Reinisch, and J.L. Coutaz, "Surface enhanced Raman scattering on silver grating: Optimized antennalike gain of the stokes signal of 10," Appl. Phys. Lett. 66, 1187, 1995.

[73] K.C. Vernon, T.J. Davis, F.H. Scholes, D.E. Gómez, and D. Lau, "Physical mechanisms behind the SERS enhancement of pyramidal pit substrates," J. Raman Spectrosc. 41, 1106-1111, 2010.

[74] X. Deng, G.B. Braun, S. Liu, P.F. Sciortino, B. Koefer, T. Tombler, and M. Moskovits, "Single-order, subwavelength resonant nanograting as a uniformly hot substrate for surface-enhanced Raman spectroscopy," Nano Lett. 10, 1780-1786, 2010.

[75] M. Jin, H. van Wolferen, H. Wormeester, A. van den Berg, and E.T. Carlen, "Large-area nanogap plasmon resonator arrays for plasmonics applications," Nanoscale 4, 4712-8, 2012. 


\section{Chapter 3}

\section{Subwavelength metal nanowire array surfaces ${ }^{1}$}

The fabrication process of metal nanowire array surfaces, which are called subwavelength metal nanowire array surfaces, and their characterization using high-resolution scanning electron microscopy and atomic force microscopy is described. An empirical model is introduced to estimate the gap size based on the pitch of a nanopatterned silicon nitride template and thickness of deposited metal.

\footnotetext{
${ }^{1}$ Adapted from: L. Le Thi Ngoc, M. Jin, J. Wiedemair, A. van den Berg, and E.T. Carlen, "Large area metal nanowire arrays with tunable sub-20 nm nanogaps," ACS Nano 7, 5223, 2013; and J. Wiedemair, L. Le Thi Ngoc, A. van den Berg, and E.T. Carlen, "Surface-enhanced Raman spectroscopy of self-assembled monolayer conformation and spatial uniformity on silver surfaces," J. Phys. Chem. C 118, 11857, 2014.
} 


\subsection{What makes a good SERS substrate?}

Typically surface-enhanced Raman scattering (SERS) substrates are realized in the form of colloidal metal nanoparticle suspensions or nanopatterned metal surfaces. Most SERS substrates are made of gold or silver because these materials support surface plasmon resonances in the visible and near infrared frequency range. In general, silver SERS substrates generate higher enhancements compared to gold; however, gold is advantageous because of its chemical stability and excellent biocompatibility. Substrates comprised of metal colloidal nanoparticles with various size and shape have been developed and are widely used, and have been reported to provide high enhancement factors, up to about $10^{10}$ [1] at "hotspot" regions where extremely large localized electromagnetic fields are generated by localized surface plasmon resonance (LSPR) on metal nanostructures. The challenge of using SERS substrates based on colloidal nanoparticles is the fact that the hotspots are randomly distributed, thus resulting in poor areal uniformity of the enhancement factor. Therefore, top-down fabricated nanostructured surfaces are preferred when using SERS for surface-enhanced Raman spectroscopy as an analytical technique.

Surface-enhanced Raman spectroscopy is commonly used to detect analytes at extremely low concentrations due to its highly sensitive molecular vibrations [2-5], and suitability for spectroelectrochemistry [6,7]. Therefore, SERS substrates need to provide electromagnetic enhancements in the range of $10^{6}-10^{8}$ in order to detect small amounts of target analytes with varying scattering cross sections. In addition to a large enhancement factor, the SERS substrates are required to have a high surface density of hotspot enhancement regions over large surface areas with high level stability and reproducibility. In general, a good SERS substrate meets the following specific criteria:

- Large and reproducible electromagnetic enhancement factor

- Large areal uniformity and high density of hotspot enhancement regions

- LSPR tunability within the visible/near-infrared range for optimized electromagnetic enhancement.

- Easy alignment of nanostructure with the excitation light polarization

- Reusable substrates that can be easily cleaned without cross-contamination from the previous measurements.

- Simple sample preparation

- Low manufacturing cost with small number of fabrication steps

- Suitability for integration with other analytical techniques, such as electrochemistry

\subsection{Metal nanowire arrays}

Over the past decade, there has been increasing interest in metal nanowires due to their unique 
electronic, optical, and electrochemical properties [8]. There are different types of metal nanowire devices and substrates depending on the manufacturing method, such as one dimensional quantum confinement devices [9], vertical metal nanowires arrays [10], and aligned planar metal nanowire arrays [11]. In this chapter, a new micro/nano-fabrication technology has been developed to realize aligned planar metal nanowire arrays, commonly referred to as nanogratings, with pitch dimensions down to about $100 \mathrm{~nm}$ and nanogap dimensions between adjacent metal nanowires less than $20 \mathrm{~nm}$, which are especially important for both electrical nanogap biochemical sensors [12] and the emerging field of plasmonics [13], where controllable and uniform nanogap dimensions over large areas are typically required.

There are many techniques available to fabricate planar nanowire arrays that can be broadly classified as either bottom-up or top-down. Bottom-up nanofabrication is simple and provides many high quality materials. However, suitable methods for accurate nanowire alignment are lacking, thus making this approach problematic for manufacturing large area metal nanowire arrays. Top-down nanofabrication techniques currently provide the best approach to manufacture arrays of aligned metal nanowires with sub-20 nm separation nanogap dimensions. Conventional top-down nanopatterning techniques, such as deep ultraviolet and immersion deep-ultraviolet stepper photolithography, are standard for semiconductor manufacturing. However, these techniques are expensive and accessible only to large integrated circuit manufacturers. Serial nanopatterning techniques, such as electron beam lithography and focused ion beam milling can realize feature sizes below $10 \mathrm{~nm}$. However, uniform chemical etching of polycrystalline gold and silver thin films over large areas can be problematic due to etching rate non-uniformity caused by grain size variations and poor photoresist adhesion, which manifests as rough nanostructure edges. In the case of ion beam milling metal materials, metal redeposition can be a limiting factor in machining nanoscale dimensions [14].

The new metal nanowires fabrication technology is based on a combination of conventional and well-characterized top-down microfabrication and nanofabrication steps to realize a template surface that facilitates the growth of a physical vapor deposited metal layer on a nanopatterned template surface, which limits the metal growth rate in the lateral direction between adjacent template surfaces, and allows for precise control of the separation nanogaps between adjacent nanowires that are electrically isolated. The advantage of this new technology is that extremely dense arrays of electrically isolated metal nanowires, with controllable elliptical surface shapes and sub-20 nm separation nanogaps, can be realized 
without the use of metal etching or milling steps. Furthermore, replacing the metal layer, which does not damage the template surface or dimensions, allows the reuse of the metal nanowire arrays described in this thesis.

The metal nanowire arrays produced with the fabrication technology presented here are quite different than conventional subwavelength metal gratings $[15,16]$, where the grating is formed by coating a nanostructured surface with a continuous metal thin film. Therefore, electrically isolated nanostructures separated by nanogaps are not passible, which precludes application requiring electrically isolated nanoelectrodes. Recently, a metal nanograting was reported that consists of isolated nanostructures that are separated by nanogaps but requires an oblique substrate orientation with the metal deposition source to control the nanogap spacing, and also has not been showed isolated nanoelectrodes [17]. Both gold nanowire and silver nanowire arrays have been fabricated and extensive characterization of the structure dimensions and cross sectional shape using high-resolution scanning electron microscopy (SEM) and atomic force microcopy (AFM) has been conducted. A simple model has been developed that can be used as design tool to estimate the nanogap dimensions based on the metal deposition process and template dimensions. The metal nanowire array substrates have been implemented as interdigitated nanoelectrode substrates for electrochemistry applications and as plasmonic substrates for SERS.

\subsection{Self-forming metal nanowire array surfaces}

The metal nanowire array nanofabrication technology presented in this chapter is based on a self-controlled metal deposition process that is facilitated by closely spaced isolated template surfaces realized with a nanopatterned silicon nitride $(\mathrm{SiN})$ layer formed on a structured silicon substrate. A brief process sequence is shown in Figure 3.1. First, a thin (30-40 nm) low stress SiN layer is deposited onto the silicon surface using a low-pressure chemical vapor deposition reactor. A $100 \mathrm{~nm}$ thick polymethyl methacrylate (PMMA, MicroChem Corp.) electron-sensitive photoresist is then spin-coated on the SiN surface and exposed to a $130 \mathrm{pA}$ electron beam with the area dose in the range of 90-120 $\mu \mathrm{As} \mathrm{cm}^{-2}$ (FEI Sirion UHR-SEM). The electron-beam exposure along the length of the SiN template was aligned to the [110] direction of the (100) silicon wafers using the wafer flat as a reference. The total template surface array area $\left(1 \mathrm{~mm}^{2}\right)$ is patterned in 100 sections each with an area of $100 \times 100 \mu \mathrm{m}^{2}$. The exposed regions are developed in a 1:3 methyl isobutyl ketone:isopropanol solution for $30 \mathrm{~s}$, and followed by immersion in isopropyl alcohol. The exposed $\mathrm{SiN}$ regions are removed using reactive ion etching $\left(60 \mathrm{~W}, 25 \mathrm{sccm} \mathrm{CHF}_{3}\right.$ and $5 \mathrm{sccm} \mathrm{O}_{2}$ ) and followed by removal of the remaining PMMA and surface cleaning with oxygen plasma (Fig. 3.1(a)). Prior to silicon 
etching, the native oxide on the exposed silicon regions is removed by immersion in $1 \%$ hydrofluoric acid solution for $1 \mathrm{~min}$. and subsequently rinsed with deionized water. The silicon is etched in a $1 \% \mathrm{KOH}$ solution at $55{ }^{\circ} \mathrm{C}$ with stirring for $45 \mathrm{~s}$ and rinsed with deionized water for 2 minutes. The different crystal planes etch anisotropically by hydroxide ions in an alkaline solution where (111) planes have the lowest etch rate, i.e. 10 times less, and (100) and (110) planes both have higher etch rates. The surfaces are then cleaned in a 3:1 piranha solution $\left(\mathrm{H}_{2} \mathrm{SO}_{4}: \mathrm{H}_{2} \mathrm{O}_{2}\right)$ for $15 \mathrm{~min}$, rinsed with deionized water for $2 \mathrm{~min}$, and dried with $\mathrm{N}_{2}$ (Fig. 3.1(b)). The resulting structure is a template surface that facilitates the growth of individual metal nanowires along the length of the $\mathrm{SiN}$ template $(y$ direction) while restricting the lateral ( $x$ direction) growth of the metal due to the isolation regions of width $w$. Conventional physical vapor deposition methods, such as sputtering or vacuum evaporation, are used to deposit the polycrystalline metal layers. The undercut sections prevent the metal from forming a continuous layer, thus the individual nanowires are similar to ultra-long cylindrical nanoparticles. The edge of the etched SiN template layer induces internal stress in the growing metal layer, which results in the elliptically shaped upper surface
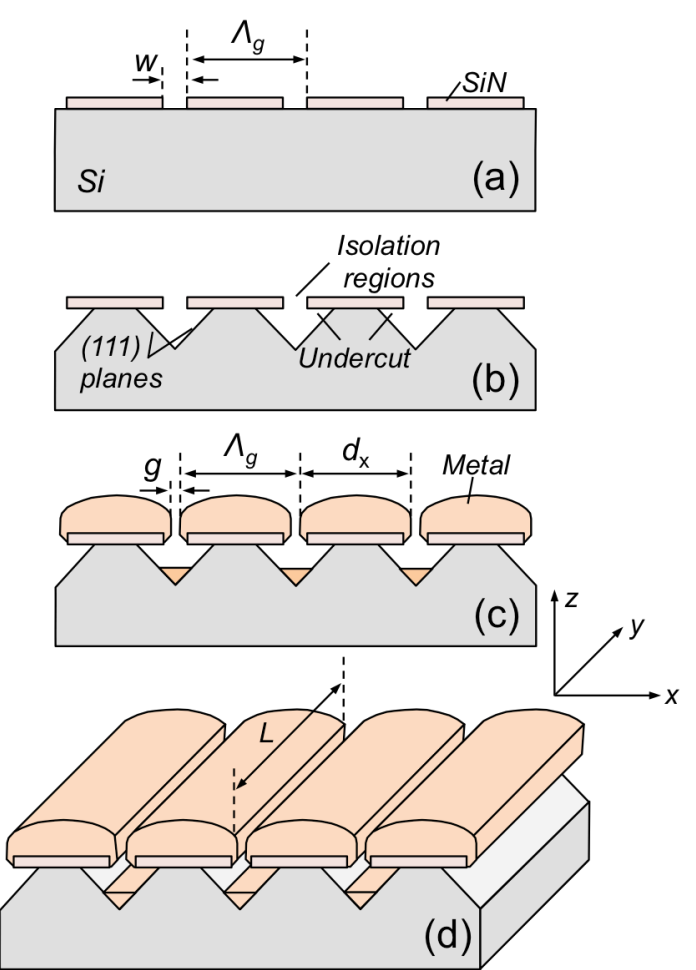

Figure 3.1. Top-down metal nanowire array fabrication procedure and relevant dimensions (not to scale). (a) Lithographically patterned and etched SiN template layer, (b) Anisotropic etching of silicon substrate to create the undercut regions, (c) Metal deposition using conventional physical vapor deposition, and (d) metal nanowire array surface. of each nanowire in the array (Fig. 3.1(c)).

The elliptical shape of the nanowire cross section, the major and the minor radii, is controlled by the metal layer thickness $t_{\mathrm{z}}$ and template pitch $\Lambda_{\mathrm{g}}$. This simple fabrication method can provide very uniform and high-density $\left(10^{5} \mathrm{~cm}^{-2}\right)$ metal nanowire arrays with small diameters (low limit, $d_{\mathrm{x}} \approx 50 \mathrm{~nm}$ ), small separation nanogaps (low limit, $g \approx 5 \mathrm{~nm}$ ), large length to pitch ratio $\left(L / \Lambda_{\mathrm{g}}>10^{4}\right)$, and extremely large nanogap length density $\left(1 \mathrm{~km} \mathrm{~cm}^{2}\right)($ Fig. $3.1(\mathrm{~d}))$ without the use of etching or milling the metal layer.

\subsection{Surface characterization}

Figure 3.2 shows high-resolution SEM images of a gold nanowire array surface fabricated 
using the procedure above, which clearly demonstrate high quality uniform metal nanowire arrays with well-controlled sub-20 nm separation gaps (Fig. 3.2(a)). Figure 3.2(b) shows an SEM image of a gold nanowire array cross section that is prepared with focused ion beam milling. The image shows that electrically isolated gold nanowires with uniform shape and sub-20 nm separation nanogap can be realized. Silver nanowire arrays have also been realized with this fabrication technique and have similar characteristics of the gold nanowire arrays.
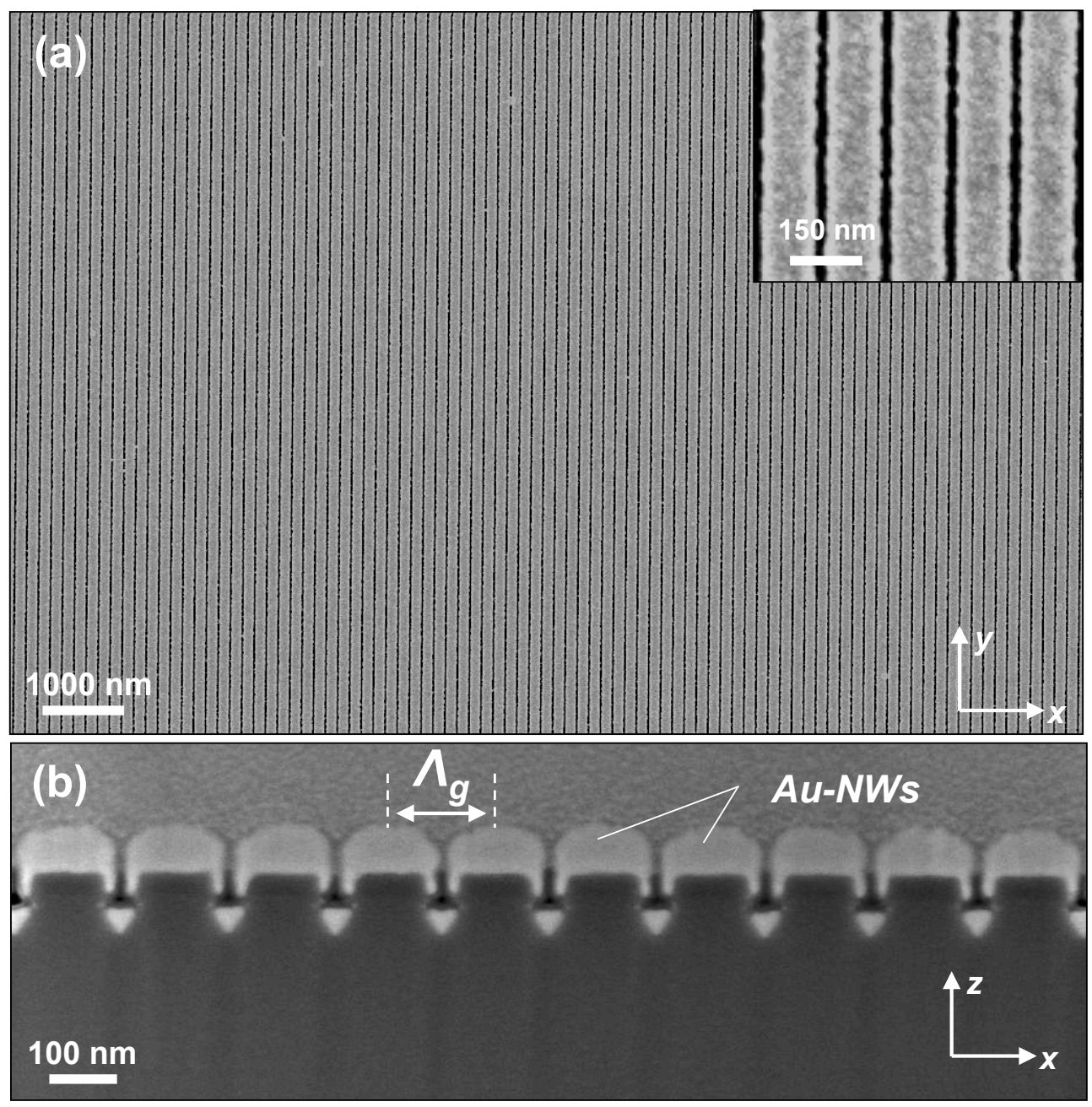

Figure 3.2. High-resolution SEM images of fabricated gold nanowire arrays. (a) Large area $(15 \times 15$ $\mu \mathrm{m}^{2}$ ) nanowire array with $\Lambda_{\mathrm{g}}=150 \mu \mathrm{m}$ pitch and $g \approx 20 \mathrm{~nm}$ nanogap spacing, (b) Gold nanowire array cross section with $\Lambda_{\mathrm{g}}=150 \mu \mathrm{m}$ and $g \approx 10 \mathrm{~nm}$, (c) High-resolution SEM mage of fabricated silver nanowire array $\Lambda_{\mathrm{g}}=200 \mu \mathrm{m}$ pitch and $g \approx 20 \mathrm{~nm}$ nanogap spacing.

The small triangular metal at the base of the isolation pit results from the deposition method, and is physically disconnected from the adjacent nanowires located on the template surface. Figure 3.3 shows representative SEM cross sections and high-resolution AFM threedimensional topography images of a gold nanowire array with $\Lambda_{\mathrm{g}}=150 \mathrm{~nm}$ (Fig. 3.3(c)) and $\Lambda_{\mathrm{g}}=100 \mathrm{~nm}$ (Fig. 3.3(d)). The nanowire shape and separation nanogap are remarkably uniform considering that a conventional physical vapor deposition method is used. The separation gap in this case is $g \approx 10 \mathrm{~nm}$ is quite well controlled. Figure 3.3(b) shows experimental 
measurements of nanogap width $g$ for different surface template separation widths $w$, and nominal metal thickness $t_{\mathrm{z}}$. The nanogap width decreases rapidly during the initial deposition of about $20 \mathrm{~nm}$ and then reduces as $g$ becomes smaller. The measured nanogap widths $g$ can be estimated with an empirical relationship $g=w \exp \left(-\alpha t_{\mathrm{z}}\right)$, where $\alpha \approx 0.02 \mathrm{~nm}^{-1}$ is the nanogap reduction parameter, which has been determined by fitting to the measured template width $\mathrm{w}$ and nominal thickness $t_{\mathrm{z}}$, shown in Fig. 3.3(b). Figure 3.3(c) clearly illustrates that the metal nanowires with sub-20 nm nanogaps are physically isolated. From the structure dimensions shown in Fig. 3.3(a), the lateral metal overlap thickness of the deposited metal layer can be estimated with $2 t_{\mathrm{x}}=w\left[1-\exp \left(-\alpha t_{\mathrm{z}}\right)\right]$, which can be used to design the separation gaps between adjacent nanowires in the array.
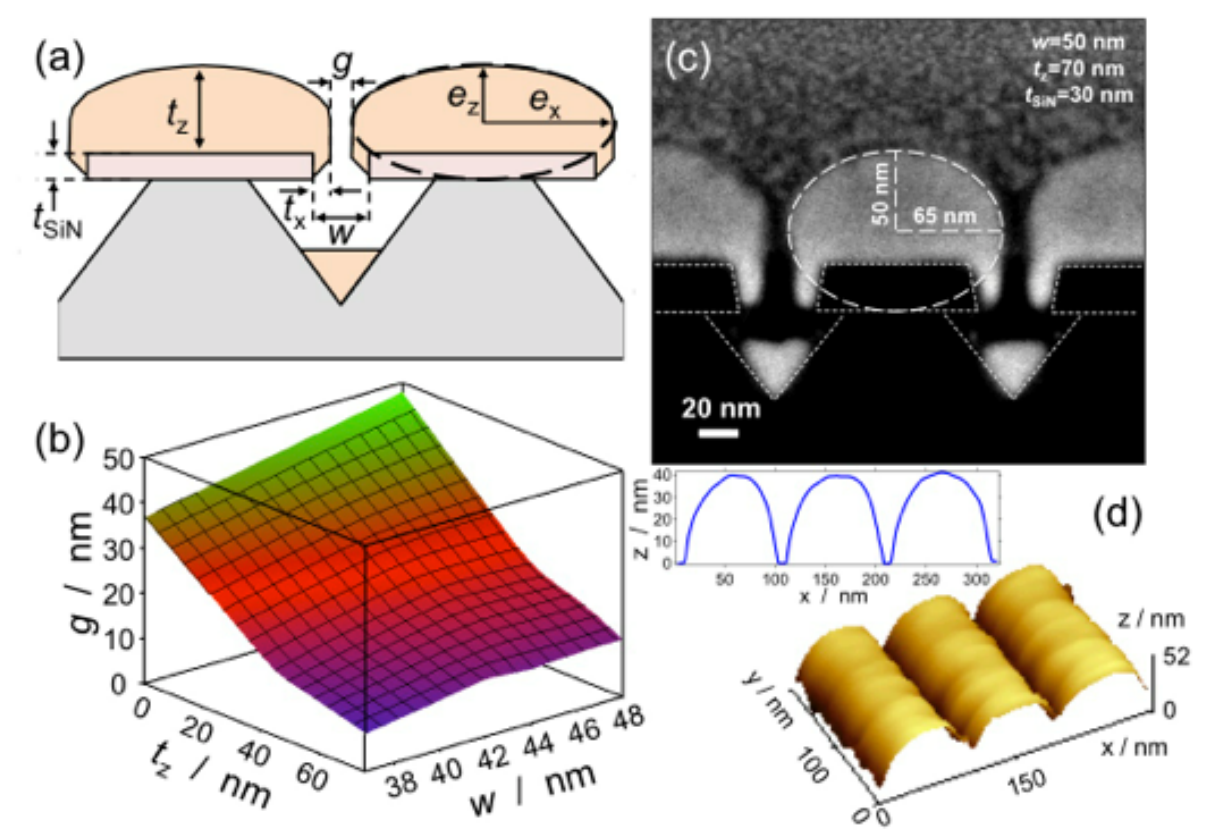

Figure 3.3. Gold nanowire array surface nanogap and shape. (a) Nanowire array cross section schematic with characteristic dimensions. Black dashed line shows the ellipse major radius $e_{\mathrm{x}}$ and minor radius $e_{\mathrm{z}}$, (b) Measured nanogap width $g$ as a function of the nominal gold thickness $t_{\mathrm{z}}$ and the template isolation width $w$, (c) High resolution SEM image of gold nanowire array cross section prepared with focused ion beam milling with $\Lambda_{\mathrm{g}}=150 \mathrm{~nm}$ and $g \approx 10 \mathrm{~nm}$. White dotted lines indicate template cross section profile (i.e., $\mathrm{SiN}$ and silicon layers). White dashed lines indicate the elliptical surface profile with $e_{\mathrm{z}}=50 \mathrm{~nm}$ and $e_{\mathrm{x}}=65 \mathrm{~nm}$, (d) High-resolution three-dimensional AFM image of upper surface and corresponding two-dimensional profile (inset) of a gold nanowire array with $\Lambda_{\mathrm{g}}=100$ $\mathrm{nm}$ and $g \approx 10 \mathrm{~nm}$.

The metal nanowire array surface follows an elliptical shape with major radius $e_{\mathrm{x}}$ and minor radius $e_{\mathrm{z}}$, as shown in Fig. 3.3(c). The major radius can be estimated with $2 e_{\mathrm{x}}$ $=\Lambda_{\mathrm{g}}-w \exp \left(-\alpha t_{\mathrm{z}}\right)$, and the minor radius estimated with $2 e_{\mathrm{z}}=t_{\mathrm{z}}+t_{\mathrm{SiN}}$, where $t_{\mathrm{SiN}}$ is the thickness of SiN layer, $\Lambda_{\mathrm{g}}$ and $w$ are defined by the template patterning method (Fig. 3.1(a)), and $t_{\mathrm{z}}$ is the nominal metal thickness, all of which can be well-controlled. Interestingly, as $\Lambda_{\mathrm{g}}$ is reduced in this case, the nanowire cross section approaches a circular shape, $e_{\mathrm{z}} \approx e_{\mathrm{x}}$, which for 
an elliptical cross section with tunable major and minor dimensions. From Fig. 3.3(c), the measured minor radius $e_{\mathrm{z}}=50 \mathrm{~nm}$ corresponds well with $\left(t_{\mathrm{z}}+t_{\mathrm{SiN}}\right) / 2$, where $t_{\mathrm{z}}=70 \mathrm{~nm}$ and $t_{\mathrm{SiN}}=30 \mathrm{~nm}$, which were determined with separate AFM measurements. With $\Lambda_{\mathrm{g}}=150 \mathrm{~nm}$, $w=50 \mathrm{~nm}, t_{\mathrm{z}}=70 \mathrm{~nm}$ and $\alpha=0.06 \mathrm{~nm}^{-1}$ corresponds to the measured major radius of $65 \mathrm{~nm}$ (Fig. 3.3(c)). Figure 3.4 shows the SEM images of gold nanowire arrays with different pitches ranging from $\Lambda_{\mathrm{g}}=100 \mathrm{~nm}$ to $\Lambda_{\mathrm{g}}=250 \mathrm{~nm}$ (Fig. 3.4(d)), and different gap widths ranging from $g=20 \mathrm{~nm}$ (Fig. 3.4(a)) to $g=10 \mathrm{~nm}$ (Fig. 3.4(d)).

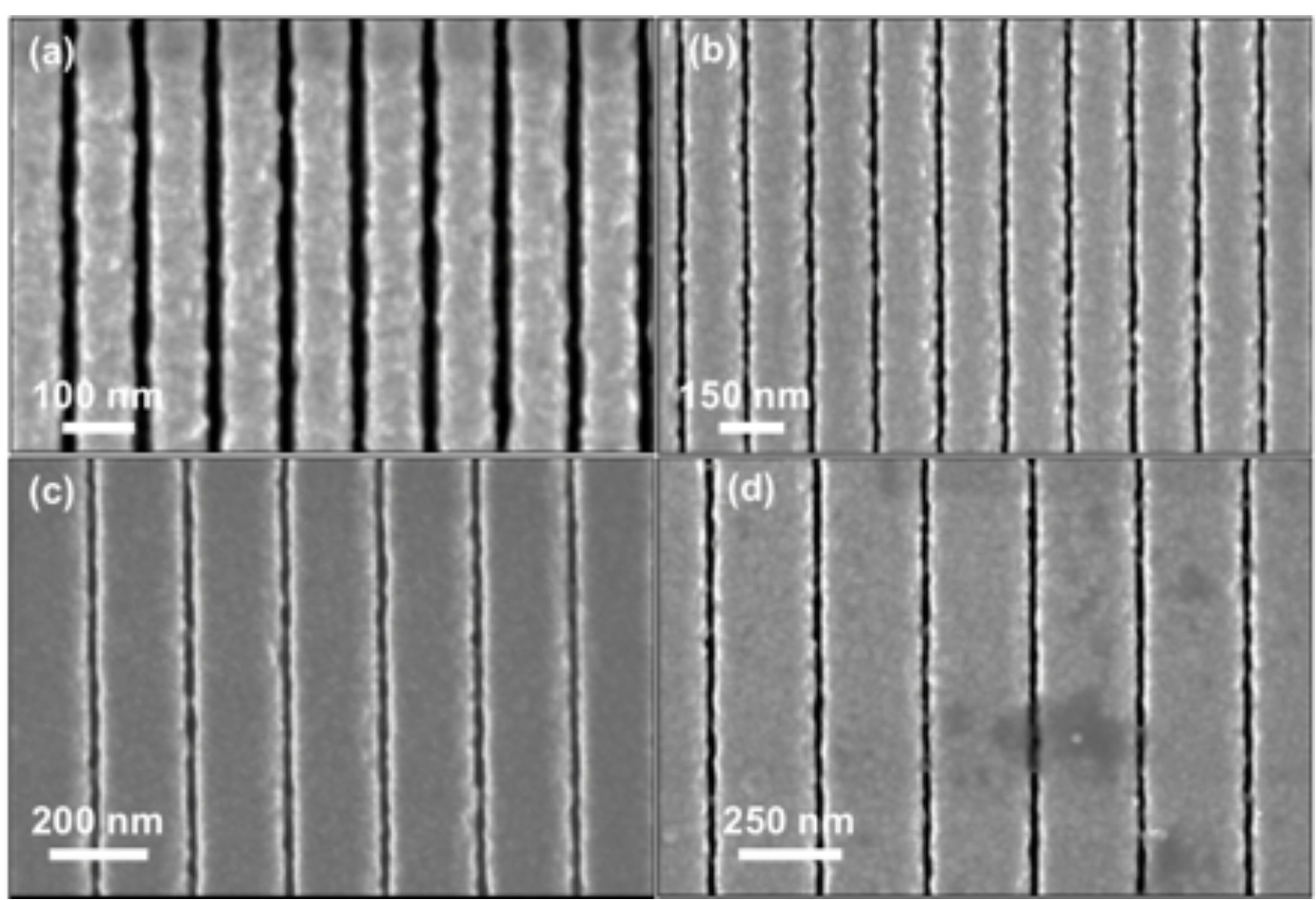

Figure 3.4. High-resolution SEM images of fabricated gold nanowire arrays with tunable pitch $\lambda_{\mathrm{g}}$ and nanogap spacing $g$. (a) $\Lambda_{\mathrm{g}}=100 \mathrm{~nm}, g=20 \mathrm{~nm}$, (b) $\Lambda_{\mathrm{g}}=150 \mathrm{~nm}, g=10 \mathrm{~nm}$, (c) $\Lambda_{\mathrm{g}}=200 \mathrm{~nm}, g=10 \mathrm{~nm}$, (d) $\Lambda_{\mathrm{g}}=250 \mathrm{~nm}, g=10 \mathrm{~nm}$.

Since the nanowire dimensions and separation nanogaps can be well controlled, the fabrication technique provides isolated metal nanostructures that have similar shape and dimensions of conventional metal cylindrical nanoparticles. However, the top-down fabrication approach results in high-density well-aligned nanowire array self-forming with sub-20 nm nanogaps. This also provides isolated nanostructures without the use of metal etching or milling which is problematic for most polycrystalline noble metal due to nonuniform material etching.

\subsection{Conclusions}

A new metal nanowire fabrication technology has been presented that is based on a combination of conventional microfabrication and nanofabrication steps to realize a template surface that eliminates the growth rate of physical vapor deposited metal layers in the lateral 
direction between adjacent template surfaces and allows for precise control of the separation nanogaps between adjacent nanowires that are electrically isolated. The advantage of this new technology is that extremely dense arrays of electrically isolated metal nanowires that have controllable elliptical surface shapes and sub-20 nm separation nanogaps can be realized without the use of a metal etching or milling step. Additionally, the template surface can be reused by selectively replacing the metal layer. The new fabrication technology using gold and silver noble metals deposited has been demonstrated with conventional physical vapor deposition have a high degree of uniformity and dimension control using far-field reflectance measurements of the nanograting structure. Finally, gold and silver nanowire arrays were fabricated and with the use of high-resolution SEM imaging to determine the nanogap dimensions, the localized surface plasmon resonance in the nanogap has been systematically tuned to common excitation laser wavelengths, $633 \mathrm{~nm}$ and $532 \mathrm{~nm}$. The metal nanowire array surfaces described in this chapter can be manufactured in any conventional microfabrication cleanroom using the established design rules.

\section{References}

[1] M. Xue, Z. Zhang, N. Zhu, F. Wang, X.S. Zhao, and T. Cao, "Transfer printing of metal nanoparticles with controllable dimensions, placement, and reproducible surface-enhanced Raman scattering effects," Langmuir, 25, 4347-4351, 2009.

[2] M. Fleischmann, P.J. Hendra, and A.J. McQuillan, "Raman spectra of pyridine adsorbed at a silver electrode," Chem. Phys. Lett. 26, 2-5, 1974.

[3] D.L. Jeanmaire and R.P. Van Duyne, "Surface Raman spectroelectro-chemistry Part I. Heterocyclic, aromatic, and aliphatic amines adsorbed on the anodized silver electrode," $J$. Electroanal. Chem. 84, 1-20, 1977.

[4] K. Kneipp, H. Kneipp, I. Itzkan, R.R. Dasari, and M.S. Feld, "Ultrasensitive chemical analysis by Raman spectroscopy," Chem. Rev. 99, 2957-2976, 1999.

[5] E.C. Le $\mathrm{Ru}$ and P.G. Etchegoin, "Single-molecule surface-enhanced Raman spectroscopy," Annu. Rev. Phys. Chem. 63, 65-87, 2012.

[6] B. Sharma, R.R. Frontiera, A.-I. Henry, E. Ringe, and R.P. Van Duyne, "SERS: Materials, applications, and the future," Mater. Today 15, 16-25, 2012.

[7] W.F. Paxton, S.L. Kleinman, A.N. Basuray, J.F. Stoddart, and R.P. Van Duyne, "Surfaceenhanced Raman spectroelectrochemistry of TTF-modified self-assembled monolayers," Phys. Chem Lett. 2, 1145-1149, 2011.

[8] Y. Xia, P. Yang, Y. Sun, Y. Wu, B. Mayers, B. Gates, Y. Yin, F. Kim, and H. Yan, "Onedimensional nanostructures: Synthesis, characterization, and applications," Adv. Mater. 15, 353-389, 2003.

[9] N. Agraït, A.L. Yeyati, and J.M. van Ruitenbeek, "Quantum properties of atomic-sized conductors," Phys. Rep. 377, 81-279, 2003.

[10] M. Kast, P. Schroeder, Y.J. Hyun, P. Pongratz, and H. Bruückl, "Synthesis of singlecrystalline Zn metal nanowires utilizing cold-wall physical vapor deposition," Nano Lett. 7, 2540-2544, 2007. 
[11] C. Xiang, S. Kung, D.K. Taggart, F. Yang, M.A. Thompson, A.G. Gu, Y. Yang, and R.M. Penner, "Lithographically patterned nanowire electrodeposition: A method for patterning electrically continuous metal nanowires on dielectrics," ACS Nano 2, 19391949, 2008.

[12] X. Chen, Z. Guo, G.-M. Yang, J. Li, M.-Q. Li, J.-H. Liu, and X.-J. Huang, "Electrical nanogap devices for biosensing," Mater. Today 13, 28-41, 2010.

[13] S.A. Maier and H.A. Atwater, "Plasmonics: Localization and guiding of electromagnetic energy in metal/dielectric structures," J. Appl. Phys. 98, 011101, 2005.

[14] K.P. Müller, "Ga+-ion beams on gold: Sputter yields and redeposition," Microelectron. Eng. 11, 443-447, 1990.

[15] H. Raether, Surface Plasmons on Smooth and Rough Surfaces and on Gratings. Springer, 1988, p. 136.

[16] X. Deng, G.B. Braun, S. Liu, P.F. Sciortino, B. Koefer, T. Tombler, and M. Moskovits, "Single-order, subwavelength resonant nanograting as a uniformly hot substrate for surface-enhanced Raman spectroscopy," Nano Lett. 10, 1780-1786, 2010.

[17] T. Siegfried, Y. Ekinci, H.H. Solak, O.J.F. Martin, and H. Sigg, "Fabrication of sub$10 \mathrm{~nm}$ gap arrays over large areas for plasmonic sensors," Appl. Phys. Lett. 99, 263302, 2011. 


\section{Chapter 4}

\section{Optical characterization of metal nanowire array surfaces ${ }^{1}$}

The optical characterization of the metal nanowire array surfaces using reflection spectroscopy and surface-enhanced Raman spectroscopy is presented. Reflection spectroscopy is used to demonstrate the plasmonic functionality, and tunability and uniformity of the dimensions of the metal nanowire array surfaces, pitch and nanogap spacing, with a normally incident linearly polarized white light source that is aligned perpendicular to the nanogaps. Surface-enhanced Raman spectroscopy is used to assess the uniformity of the electromagnetic enhancement of the gold nanowire array surfaces by covering the array surfaces with a benzenethiol self-assembled monolayer, which provides an estimation of the uniformity of the spatially averaged enhancement factor from a small number of molecules.

\footnotetext{
${ }^{1}$ Adapted from: L. Le Thi Ngoc, M. Jin, J. Wiedemair, A. van den Berg, and E.T. Carlen, "Large area metal nanowire arrays with tunable sub-20 nm nanogaps," ACS Nano 7, 5223, 2013; and J. Wiedemair, L. Le Thi Ngoc, A. van den Berg, and E.T. Carlen, "Surface-enhanced Raman spectroscopy of self-assembled monolayer conformation and spatial uniformity on silver surfaces," J. Phys. Chem. C 118, 11857, 2014.
} 


\subsection{Plasmonics: volume plasmons, surface plasmons, localized surface plasmons, and gap plasmons}

The emerging field of plasmonics, which has been defined as the science and engineering of surface plasmon polariton excitation on conductor-dielectric interfaces [1], has grown steadily over the last half century since the discovery of bulk plasmons [2], and surface plasmon polaritons on noble metal foils [3]. Interest in surface plasmons, continued to grow due to the discoveries of surface-enhanced Raman scattering (SERS) on electrochemically roughened silver surfaces $[4,5]$, second harmonic generation (SHG) on metal surfaces [6], and later the development and commercialization of surface plasmon resonance biosensors on planar metal surfaces [7]. More recently, surface-enhanced resonance Raman scattering (SERRS) spectra from single molecules have been reported $[8,9]$. Today, the unique properties of surface plasmon resonances and plasmonic-based devices are being applied to label-free biosensing, surface-enhanced Raman spectroscopy, nanoscale optical components, energy conversion, and quantum cascade lasers. Over this period of time several key technological advancements have aided its steady growth, such as three-dimensional simulation and analysis tools, high precision nanofabrication and materials synthesis techniques, and high quality lasers and optical components, all of which provide scientists and engineers with the tools necessary for designing, fabricating, and analyzing the optical behavior of metal nanostructures.

A plasmon is a quasi-particle representing the quantum of collective plasma ${ }^{2}$ oscillations in a free electron gas; Pines and Bohm [2,10] identified that the long-range Coulomb interaction between valence electrons in metals yields collective plasma oscillations similar to the electron density oscillations gaseous plasmas as reported by Langmuir [11], which are both longitudinal oscillations. The collective longitudinal oscillations of conduction electrons, with respect to the fixed positive ion cores, are called volume plasmons. Due to the longitudinal nature of the volume plasmon oscillations, they cannot be excited with transverse electromagnetic waves, but have been excited with focused electron beams [2]. The quanta of the volume plasmons have an oscillation frequency defined as

$$
\omega_{p}=\left(\frac{n q^{2}}{\varepsilon_{0} m_{0}}\right)^{1 / 2},
$$

where $n$ is the number of free electrons per unit volume, $\varepsilon_{0}$ is the permittivity of free space, and $m_{0}$ is the free electron mass. For gold, $n \approx 5.9 \times 10^{28} \mathrm{~m}^{-3}$, which gives $\omega_{\mathrm{p}} \approx 1.38 \times 10^{16} \mathrm{rad} \mathrm{s}^{-1}$ and $\lambda_{\mathrm{p}} \approx 2 \pi c / \omega_{\mathrm{p}}=137 \mathrm{~nm}$, and for silver, $n \approx 5.86 \times 10^{28} \mathrm{~m}^{-3}$, which gives $\omega_{\mathrm{p}} \approx 1.37 \times 10^{16} \mathrm{rad} \mathrm{s}^{-1}$

\footnotetext{
${ }^{2}$ Plasma is a medium with an equal concentration of positive and negative charges, where at least one type is mobile. In the solid state, the negative charges of the conduction electrons are balanced by the positive ion cores [12].
} 
and $\lambda_{\mathrm{p}} \approx 2 \pi c / \omega_{\mathrm{p}}=137 \mathrm{~nm}$. The volume plasmons decay through energy transfer to single electrons, which is known as Landau damping [13], thus behave as a relaxator system [14].

A few years after the discovery of volume plasmons, the existence of longitudinal surface collective charge density oscillations at a metal-dielectric interface was predicted to occur on thin metal foils [3], which was subsequently demonstrated with electron energy-loss experiments [15,16], and were subsequently called the surface plasmons polaritons [17]. Surface plasmons polaritons ${ }^{3}$, or surface plasmons, excited on metal surfaces are electromagnetic waves of collective electron oscillations that are confined to the interface between a non-absorbing dielectric material with positive real dielectric constant $\varepsilon_{\mathrm{d}}{ }^{4}$ and a conducting surface, such as a metal, with negative real value of the dielectric function $\varepsilon_{\mathrm{m}}(\omega)$ with energy proportional to $\hbar \omega_{\mathrm{p}} \eta^{-1}$, where $\eta$ is a scaling factor that depends on the shape of the nanostructure, and the dielectric properties of the metal $\varepsilon_{\mathrm{m}}(\omega)$ and the surrounding medium $\varepsilon_{\mathrm{d}}$.

Surface plasmons can take the form of propagating electromagnetic waves on planar surfaces that are confined in one dimension normal to the interface (Fig. 4.1(a)), or localized surface plasmon resonances on curved nanoscale structures where the surface fields are confined in two dimensions, for example, cylindrical nanostructures (Fig. 4.1(b)) and in three dimensions, for example, spherical nanostructures (Fig. 4.1(c)). The frequency of the surface plasmons on planar interfaces is linked to the wavevector $k_{\mathrm{spp}}$ through a dispersion relation $k_{\mathrm{spp}}(\omega)$, where $\omega$ is the angular frequency. These charge oscillations are localized in the $z$ direction (Fig. 4.1(a)) and are accompanied by a mixed transverse and longitudinal electromagnetic wave $\boldsymbol{E}_{\mathrm{s}}=\boldsymbol{E} \exp \left[i\left(k_{\mathrm{spp}} x \pm k_{\mathrm{z}} z-\omega t\right)\right]$ with imaginary $k_{\mathrm{z}}$, which results in an exponential decay of the $z$-component of the electromagnetic field $\boldsymbol{E}_{s z} \sim \exp \left[-\left|k_{z}\right| z\right]$ (Fig. 4.1(a)). The wavevector $k_{\mathrm{spp}}$ lies parallel to the metal-dielectric interface ( $x$-direction) with a wavelength of $\lambda_{\mathrm{spp}}=2 \pi / k_{\mathrm{spp}}$.

Maxwell's equations give the dispersion relation of the surface plasmon polariton $k_{S P P}=\omega / c \sqrt{\varepsilon_{m}(\omega) \varepsilon_{d} /\left(\varepsilon_{m}(\omega)+\varepsilon_{d}\right)}$, where $c$ is the speed of light in vacuum. The surface plasmon wavevector $k_{\mathrm{spp}}$ does not cross the light line, hence is nonradiative, and the oscillation frequency for large $k_{\mathrm{spp}}$ approaches $\omega_{\mathrm{spp}}=\omega_{\mathrm{p}} /(2)^{1 / 2}$ for an air-metal interface [18]. Surface plasmons on a planar metal-dielectric interface cannot be excited directly by incident light since $k_{\mathrm{spp}}>k$, where $k(k \equiv \omega / c)$ is the wavevector of light on the dielectric side of the interface.

\footnotetext{
${ }^{3}$ A surface plasmon polariton is a quantum of a coupled plasmon-photon wave field [18].

${ }^{4}$ It is assumed that the non-absorbing dielectric material is dispersionless in the visible to near infrared wavelength range with positive real dielectric constant.
} 
Phase matching of incident light to surface plasmon polaritons can be achieved using prism coupling or grating coupling. When surface plasmon polaritons are confined to a nanostructure with a characteristic dimension $D$, such that $D<<\lambda$, where $\lambda$ is the wavelength of the incident excitation, the free electrons of the nanostructure form a collective oscillation, which is called a localized surface plasmon resonance. Figures 4.1(b) and 4.1(c) schematically show the electric near-field dipolar local surface plasmon optical response of metal nanoparticles to uniform $x$-polarized plane wave excitation.
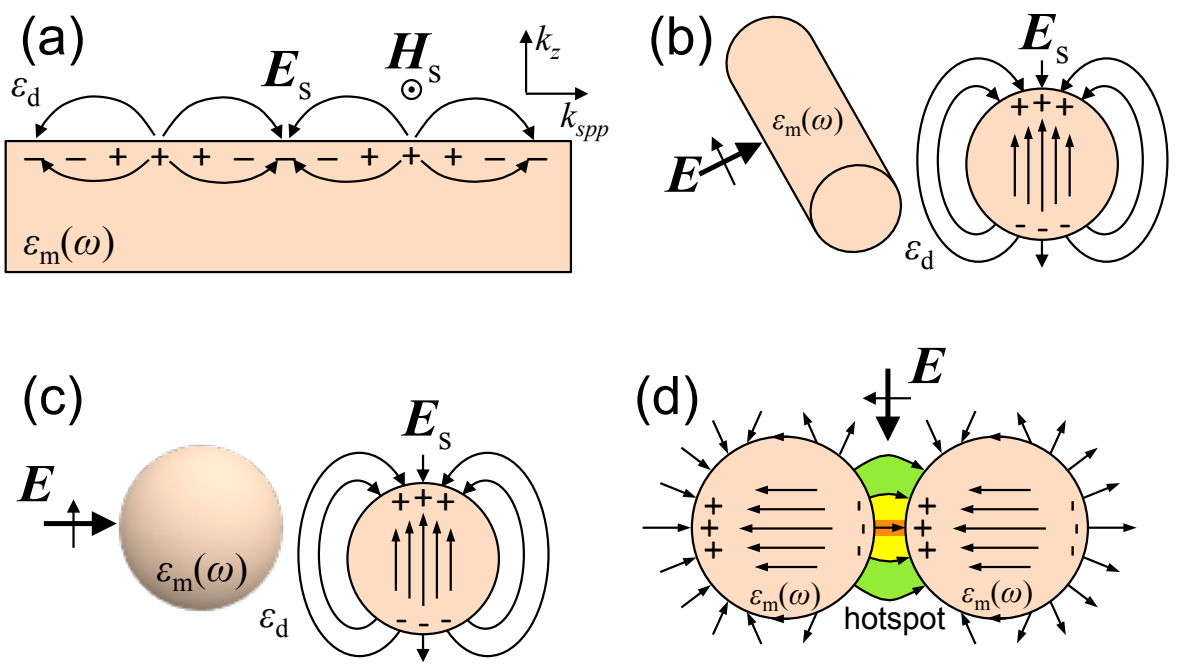

Figure 4.1. Metal-dielectric interfaces that support surface plasmon resonances. (a) 1D planar surface plasmon polariton response, (b) Dipolar localized surface plasmon resonance response of 2D infinite cylinder, (c) Dipolar localized surface plasmon resonance response of 3D sphere nanoparticle, and (d) Closely spaced spherical metal nanoparticles excited electromagnetic radiation polarized along the inter-particle axis and coupled-mode plasmon resonance hotspot.

The negative conduction electrons oscillate under the influence of the external field, against the positive ionic charges, which results in a net displacement of the negative charges. The electron displacement results in polarization charges at the curved nanoparticle surface, thus exerting a restoring force on the driven electrons and the localized surface plasmon resonance frequency of the system. Thus, the conduction electrons in the nanoparticle behave as an oscillator system, in contrast to the volume plasmons in a bulk material that behave as a relaxator system.

In order to show how the electromagnetic fields at the nanoparticle surface are affected by the localized surface plasmon resonance, spherical nanoparticle and infinite nanocylinder are typically analyzed due to their simplicity. Using the electrostatic approximation $(D<<\lambda)$, and an $x$-polarized uniform plane wave excitation $\boldsymbol{E}=E \hat{x}$ (Fig. 4.1(b)), the electric field inside an spherical nanoparticle with diameter $D=2 a$ is

$$
\boldsymbol{E}_{i n}=E \frac{3 \varepsilon_{d}}{\varepsilon_{m}(\omega)+2 \varepsilon_{d}} \hat{x}=E \frac{3 \varepsilon_{d}}{\varepsilon_{m}(\omega)+2 \varepsilon_{d}}(\cos \theta \hat{r}-\sin \theta \hat{\theta}),
$$


and the electric field in the near the surface (Fig. 4.1(b)) is ${ }^{5}$

$$
\boldsymbol{E}_{S}=E(\cos \theta \hat{r}-\sin \theta \hat{\theta})+E \frac{a^{3}}{r^{3}} \frac{\varepsilon_{m}(\omega)-\varepsilon_{d}}{\varepsilon_{m}(\omega)+2 \varepsilon_{d}}(2 \cos \theta \hat{r}+\sin \theta \hat{\theta}) .
$$

Defining the dipole moment as $\boldsymbol{p}=\varepsilon_{0} \varepsilon_{m} \alpha \boldsymbol{E}$, and using Eq. (4.3) with $\boldsymbol{E}_{S}=-\nabla V_{S}$, where $V_{\mathrm{S}}$ is the potential near the surface of the nanoparticle. The polarizability can be written as

$$
\alpha=4 \pi a^{3} \frac{\varepsilon_{m}(\omega)-\varepsilon_{d}}{\varepsilon_{m}(\omega)+2 \varepsilon_{d}} .
$$

From Eq. (4.4), the polarizability is resonantly enhanced for minimum value of $\left|\varepsilon_{\mathrm{m}}(\omega)+2 \varepsilon_{\mathrm{d}}\right|$, which simplifies to $\operatorname{Re}\left[\varepsilon_{\mathrm{m}}(\omega)\right]=-2 \varepsilon_{\mathrm{d}}$ if the metal absorption $\operatorname{Im}\left[\varepsilon_{\mathrm{m}}(\omega)\right]$ is small. This is called the Fröhlich condition for the dipole surface plasmon of the metal nanoparticle. For a Drude metal, the resonance condition is met at $\omega_{\mathrm{spp}}=\omega_{\mathrm{p}} /(3)^{1 / 2}$. Therefore, the dielectric function of the metal layer $\varepsilon_{\mathrm{m}}(\omega)$ plays a very important role in surface plasmon resonance effects. The real part of the dielectric function $\operatorname{Re}\left[\varepsilon_{\mathrm{m}}(\omega)\right]$ is responsible for the plasmon resonance and the imaginary part $\operatorname{Im}\left[\varepsilon_{\mathrm{m}}(\omega)\right]$ is responsible for absorption in the metal. Among the noble metals, silver and gold are the most commonly used for plasmonics applications since the resonance condition $\operatorname{Re}\left[\varepsilon_{\mathrm{m}}(\omega)\right]=-2 \varepsilon_{\mathrm{d}}$ is matched for $\omega$ in the visible spectrum. Using the same assumptions, the electric field inside the nanocylinder is

$$
\boldsymbol{E}_{i n}=E \frac{2 \varepsilon_{d}}{\varepsilon_{m}(\omega)+\varepsilon_{d}} \hat{x}
$$

and in the near-zone outside is

$$
\boldsymbol{E}_{S}=E \hat{x}+E \frac{\varepsilon_{m}(\omega)-\varepsilon_{d}}{\varepsilon_{m}(\omega)+\varepsilon_{d}}\left[\frac{a^{2}}{r^{2}}\left(1-2 \sin ^{2} \varphi\right) \hat{x}+2 a^{2} \sin \varphi \cos \varphi \hat{y}\right],
$$

where cylindrical coordinates have been used to simplify the expression. ${ }^{6}$ For a Drude metal, the resonance condition for the infinite nanocylinder is $\omega_{\mathrm{spp}}=\omega_{\mathrm{p}} /(2)^{1 / 2}$, which is the same as a planar surface.

More importantly, localized surface plasmon resonances generated in nanogaps located between adjacent nanostructures, called hotspots, result in coupled-mode resonances with energies that are dependent on the nanostructure shape, nanogap dimensions, and metal layer (Fig. 4.1(d)). When two spherical nanoparticles (dimers) are in close proximity where a nanogap is formed and the excitation radiation polarization is aligned along the inter-particle axis (Fig. 4.1(d)), the localized surface plasmon resonance modes of each nanosphere form hybridized modes and the electromagnetic field magnitude in the nanogap is dramatically

\footnotetext{
${ }^{5}$ Spherical coordinates are defined as $(r, \theta, \phi)$ where $x=r \sin \phi \cos \phi, y=r \sin \phi \sin \phi$, and $z=r \cos \phi$.

${ }^{6}$ Cylindrical coordinates are defined as $(r, \varphi, z)$ where $x=r \cos \varphi, y=r \sin \varphi$, and $z=z$.
} 
increased. As the distance between the metal nanoparticles is reduced, the transition dipoles composed of oscillating conduction electrons couple and classical electrodynamics predicts that the enhanced fields around each particle coherently interfere [14]. As the separation nanogap is further decreased, the coupled plasmon resonance wavelength red shifts and the electromagnetic field enhancement further increases.

In all types of surface plasmon resonances, the magnitude of the electromagnetic field near the metal nanostructure surface $\left|\boldsymbol{E}_{\mathrm{s}}\right|$, or in nanogap hotspot regions, induced at the metaldielectric interface is amplified $M(\boldsymbol{r}, \omega) \equiv\left|\boldsymbol{E}_{\mathrm{s}}(\boldsymbol{r}, \omega)\right|^{2} /|\boldsymbol{E}(\boldsymbol{r}, \omega)|^{2}$, with respect to the incident field $\boldsymbol{E}$, where enhancements in nanogap regions currently account for the largest enhancements. The electromagnetic field enhancement is an important property of a plasmonic surface for SERS applications. The magnitude and coupling energy of the electromagnetic field enhancement can be tuned for a specific application and implemented using different materials, nanostructure and nanogap geometry and dimension, which leads to one of the most important challenges in the field of plasmonics: a reliable manufacturing technology for the implementation of large area substrates with spatially uniform patterned metal nanostructures with sub-20 $\mathrm{nm}$ separation nanogaps.

\subsection{Optical properties of gold and silver}

The optical properties of metals are different from conventional dielectrics because of the presence of free electrons. The optical response of noble metals, such as gold, silver and copper, in the visible and infrared energy regime is dominated by the interaction photons and the free-electron plasmons, which are mixed photon-plasmon modes at the plasma frequency $\omega_{\mathrm{p}}$ (Eq. 4.1). The optical response of the collection of free electrons can be described by the Drude model that is based on a simplified Lorentz harmonic oscillator model, giving the dielectric function for the free electrons in the metal as

$$
\varepsilon(\omega)=1-\frac{\omega_{p}^{2}}{\omega^{2}+i \gamma \omega}
$$

where $\gamma$ is a damping constant resulting from electron-electron and electron-phonon scattering in the metal [16]. The dielectric function is composed of real and imaginary parts $\varepsilon(\omega)=\varepsilon_{1}(\omega)+i \varepsilon_{2}(\omega)$, which is factored into

$$
\begin{aligned}
& \varepsilon_{1}(\omega)=1-\frac{\omega_{p}^{2}}{\omega^{2}+\gamma^{2}}, \\
& \varepsilon_{2}(\omega)=\frac{\omega_{p}^{2} \gamma}{\omega\left(\omega^{2}+\gamma^{2}\right)} .
\end{aligned}
$$


The plasmons play a very important role in the optical properties of the metal. There is strong reflection for all frequencies below the plasmon resonance frequency $\omega_{\mathrm{p}}$. If the light frequency is lower than the plasmon frequency $\omega<\omega_{\mathrm{p}}$, the light will be reflected since electrons screen the light. For light frequencies above the plasma frequency $\omega>\omega_{\mathrm{p}}$, the light transmits through the metal because the electrons cannot react fast enough to screen it.

The dielectric function of real metals requires modification to the Drude model to account for inter-band and intra-band transitions at optical frequencies. Among the noble metals, silver and gold are the most commonly used for plasmonics applications. Inter-band transitions in real metals affect the optical response, and strongly affect the optical properties of gold in the ultraviolet-visible region of the spectrum due to separation of the $d$-band and the $6 s p$-band near the $X$ - and $L$-symmetry points in the first Brillouin zone, which results in two inter-band transitions near $1.84 \mathrm{eV}$ and $2.45 \mathrm{eV}$ [19]. The Lorentz-Drude dielectric function model is commonly used to accommodate inter-band transitions in real metals

$$
\varepsilon(\omega)=\varepsilon_{\infty}-\frac{\omega_{p}^{2}}{\omega^{2}-i \gamma_{p} \omega}+\sum_{i} \chi_{i}(\omega)
$$

where two susceptibility terms

$$
\chi_{i}(\omega)=A_{i}\left[e^{-i \theta}\left(\omega_{i}-\omega-i \gamma_{i}\right)^{-1}+e^{-i \theta}\left(\omega_{i}+\omega+i \gamma_{i}\right)^{-1}\right],
$$

are required to $\mathrm{fit}^{7}$ the data in the visible region of the spectrum to the measured data from [20]. Figure 4.2 shows measured dielectric function data of $\varepsilon_{1}(\omega)$ and $\varepsilon_{2}(\omega)$ from gold thin films from Johnson and Christy [20].

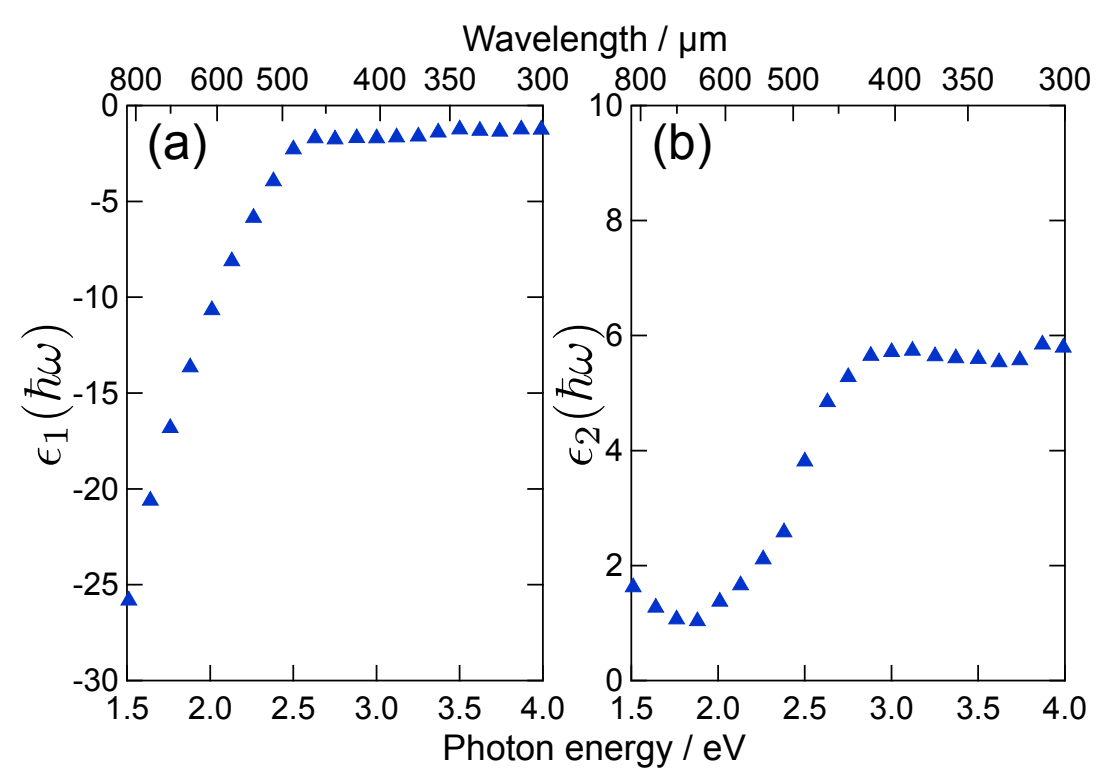

Figure 4.2. (a) Real and (b) Imaginary dielectric function data of gold films [20].

\footnotetext{
${ }^{7}$ Fit parameters are $\varepsilon_{\infty}=1.5, \omega_{\mathrm{p}}=1.37 \times 10^{16} \mathrm{~s}^{-1}, \gamma_{\mathrm{p}}=0.14 \times 10^{15} \mathrm{~s}^{-1}, A_{1}=0.4 \times 10^{15}, \gamma_{1}=1.2 \times 10^{15} \mathrm{~s}^{-1}, \omega_{1}=\Delta_{\mathrm{X}} / \hbar$, $\theta=-\pi / 2.7, A_{2}=0.95 \times 10^{16}, \gamma_{2}=1.4 \times 10^{15} \mathrm{~s}^{-1}$, and $\omega_{2}=\Delta_{\mathrm{L}} / \hbar$.
} 
The inter-band absorption in silver occurs in the ultraviolet region of the spectrum near the $L$ symmetry point in the first Brillouin zone, which is near $3.9 \mathrm{eV}$ [21]. Figure 4.3 shows measured $\varepsilon_{1}(\omega)$ and $\varepsilon_{2}(\omega)$ data from [20].

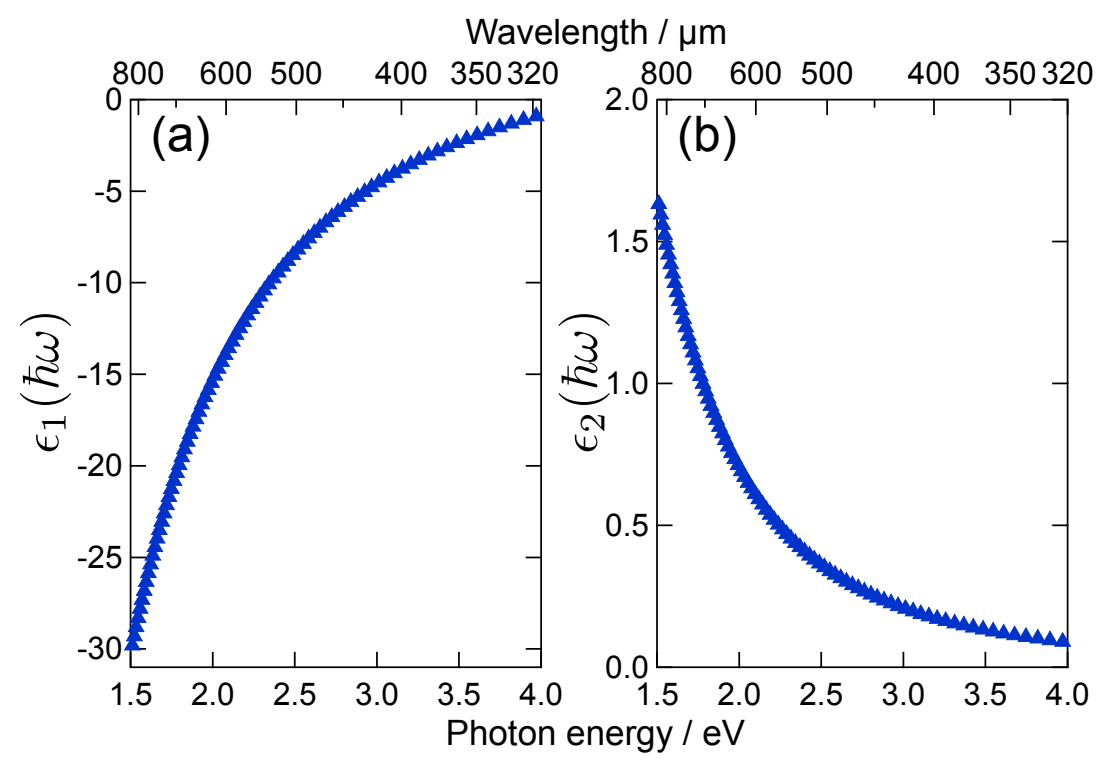

Figure 4.3. (a) Real and (b) Imaginary dielectric function data of silver films [20].

Among the noble metals, silver and gold are the most commonly used for plasmonics applications. The excitation wavelength for gold is limited to $\lambda>600 \mathrm{~nm}$, due to the strong absorption as shown in the dielectric function (Fig. 4.2). At near infrared wavelengths the optical response of gold is comparable to that of silver. However, there are other very important issues for SERS, which include availability, ease of manipulation, toxicity, and durability. Therefore, gold is the best choice for applications where $\lambda>600 \mathrm{~nm}$, and silver is the best for the applications requiring $\lambda<600 \mathrm{~nm}$.

\subsection{Plasmonic substrates}

Many different techniques have been reported to fabricate metal plasmonic substrates. Colloidal nanoparticle suspensions comprised of bottom-up synthesized metal nanoparticles of various shapes and sizes are attractive due to their preparation simplicity and crystalline structure; however, they typically have poor enhancement reproducibility, which is attributed to many factors including their random composition and lack of precise dimensional control, nanogap spacing, and excitation polarization alignment. Top-down fabricated plasmonic substrates have also been reported extensively over the last three decades. Patterning thin metal layers by using a variety of different nanolithography techniques, such as electron-beam patterning and nanoimprinting, have been used to realize plasmonic surfaces in many different configurations, including discs of many shapes and sizes [22], nanohole arrays [23], and antenna nanostructures [24]. Low-cost non-lithographic methods have been reported, 
such as electrochemically roughened surfaces, self-assembled templated colloidal films, and nanosphere lithography [25]. However, forming small and uniform separation nanogaps over large surface areas remains problematic.

Currently, the major challenge in fully exploiting coupled-mode plasmon resonances on metals is the reproducible realization of large area metal nanostructure arrays with uniform sub-20 $\mathrm{nm}$ separation nanogaps in high density, as previously described. There have been many different types of plasmonic substrates that can be broadly categorized as isolated metal nanostructure arrays and metal nanostructure arrays formed from a continuous metal layer. Isolated metal nanostructure arrays are typically realized with bottom-up fabrication techniques and come in many forms, such as colloidal nanoparticle suspensions [26] and selfassembled colloidal films [27].

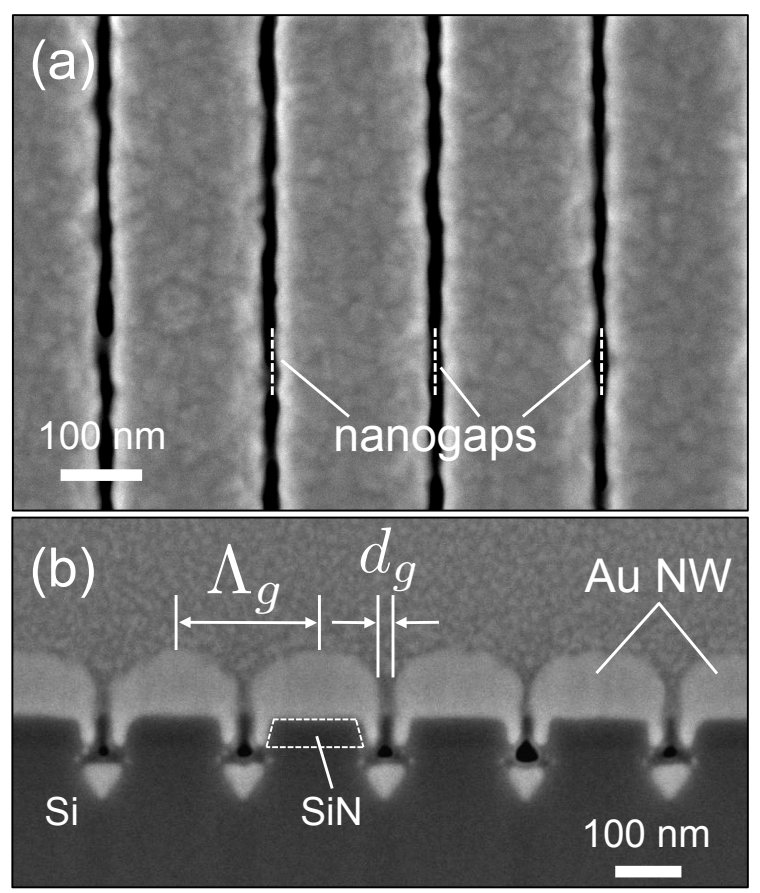

Figure 4.4. High-resolution scanning electron microscopy image of gold nanowire array surface with sub-20 nm nanogap. (a) Top view, (b) Surface cross section.

Colloidal nanoparticle suspensions and films, which are relatively simple to prepare, however, typically have poor enhancement uniformity and reproducibility due to their random orientation and composition, lack of precise dimension control, separation spacing, and excitation polarization alignment, which is especially important for coupled-mode plasmon resonances. Top-down fabrication techniques have also been employed for the realization of isolated metal nanostructure arrays, such as nanosphere lithography [25], electron-beam patterning, focused ion-beam milling, and nanoimprinting [7,22,28-30], however, the formation of well-controlled nanogaps over large areas in high density remains problematic. Continuous metal nanostructure plasmonic substrates are usually realized with top-down 
fabrication techniques, such as electrochemically-roughened surfaces [4] and surface templates coated with the active plasmonic layer [31-34], both of which have not achieved surfaces with both high nanogap density and large enhancements over large areas.

A new top-down manufacturing technique was recently developed to produce large area arrays of metal nanowires with high density array sub-20 nm separation nanogaps, shown in the scanning electron microscopy (SEM) images in Fig. 4.4, using nanopatterned siliconnitride layers to form isolated templates [35]. The nanopatterned templates enable a surface tension controlled metal deposition procedure that provides precise control of the nanogap dimensions; hence the surface plasmon excitation energy can be closely matched to the energy of the excitation laser, which provides the maximum near-field enhancement. The surfaces provide extremely large electromagnetic field enhancements and recently have been used for surface-enhanced Raman spectroscopy to assess the molecular conformation of selfassembled monolayers on silver surfaces [36] and spectroelectrochemical analysis of metalloporphyrin modified gold nanostructured electrodes [37].

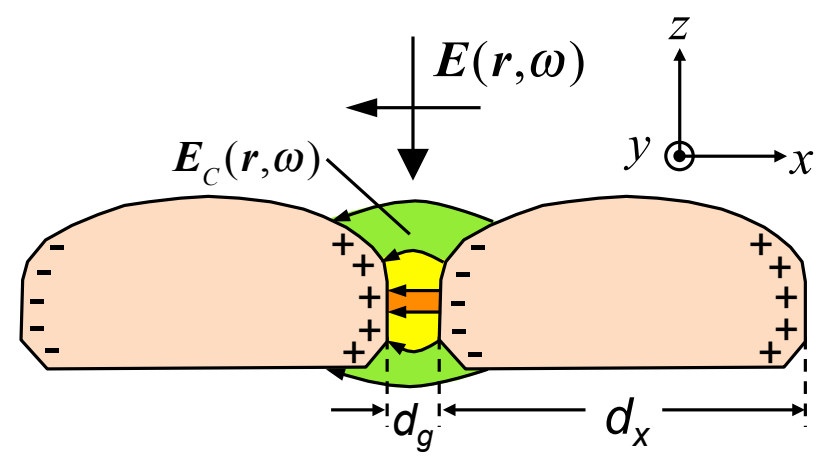

Figure 4.5. Plane wave (p-polarized) excitation $\boldsymbol{E}(\boldsymbol{r}, \boldsymbol{\omega})$ of coupled-mode local surface plasmon resonance between a closely spaced metal-nanowires and resulting coupled electromagnetic field $\boldsymbol{E}_{\mathrm{c}}(\boldsymbol{r}, \omega)$.

The metal nanowire array surfaces are well suited for plasmonics applications, which use metal nanostructures that couple far-field electromagnetic radiation into localized surface plasmon modes that are confined to the interface of a non-absorbing dielectric, with positive real dielectric constant, to a conductive material with negative real value of its dielectric function, which is typically a noble metal. Figure 4.5 shows a schematic of the cross section of the metal nanowire array surface. The generation of the localized surface plasmon resonance on the nanowire dimer pair in close proximity with $d_{\mathrm{g}}<d_{\mathrm{x}}$, the enhanced electromagnetic fields around each nanostructure coherently interfere, thus resulting in a coupled plasmon electromagnetic field $\boldsymbol{E}_{\mathrm{c}}(\boldsymbol{r}, \omega)$ in the nanogap, which results in an increase in the electromagnetic field enhancement $M_{\mathrm{c}}(\boldsymbol{r}, \omega)=\left|\boldsymbol{E}_{\mathrm{c}}(\boldsymbol{r}, \omega)\right|^{2} /|\boldsymbol{E}(\boldsymbol{r}, \omega)|^{2}$, when the excitation polarization is aligned along the inter-particle axis. For small nanogaps, where $d_{\mathrm{g}}<20 \mathrm{~nm}$, the 
coupled-plasmon enhancement increases rapidly and typically results in $M_{\mathrm{c}}>>M_{\mathrm{s}}[38,39]$.

The coupled-mode plasmon resonators have important applications in surface spectroscopy [40], fluorescence enhancement [41], energy conversion[42], and nanoscale optics [43].

\subsection{Reflectance spectroscopy}

Reflection spectroscopy is used to demonstrate the plasmonic functionality, and tunability and uniformity of the dimensions of the metal nanowire array surfaces, pitch $\Lambda_{\mathrm{g}}$ and nanogap $d_{\mathrm{g}}$, with a normally incident linearly p-polarized white light source that is aligned perpendicular to the nanogaps. Figure 4.6 shows examples of reflectance measurements of gold nanowire and silver nanowire array surfaces where the reflectance decreases to a minimum value at a certain wavelength that corresponds to the localized surface plasmon resonance wavelength $\lambda_{\mathrm{L}}$. For all measurements a planar gold, or silver, surface is used as a reflectance reference. Figures 4.6(a), 4.6(b), and 4.6(c) show that the localized surface plasmon resonance wavelength (reflectance minimum) of gold nanowire array surfaces with different $\Lambda_{\mathrm{g}}$ and $g$ (realized using different gold thickness $t_{\mathrm{z}}$ ) can be precisely tuned to $633 \mathrm{~nm}$ and $785 \mathrm{~nm}$, commonly used laser wavelengths used in visible spectrum plasmonics applications.

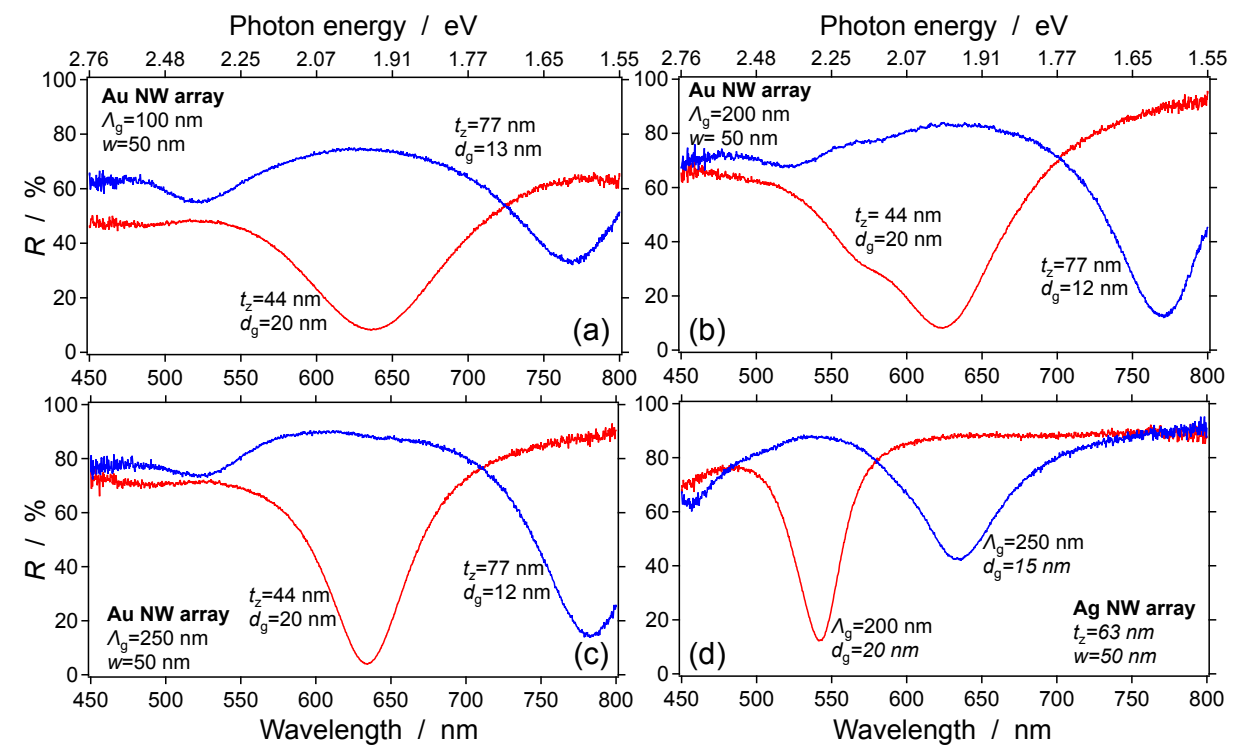

Figure 4.6. Measured reflectance of gold nanowire and silver nanowire array surfaces with different pitch $\Lambda_{\mathrm{g}}$ and nanogap width $d_{\mathrm{g}}$ showing the localized surface plasmon resonance tuned to commonly used laser wavelengths $\left(\lambda_{\mathrm{L}}=532 \mathrm{~nm}, 633 \mathrm{~nm}\right.$ and $\left.785 \mathrm{~nm}\right)$. (a) Gold nanowire with $\Lambda_{\mathrm{g}}=100 \mathrm{~nm}$ and $w=50 \mathrm{~nm}$, (b) Gold nanowire with $\Lambda_{\mathrm{g}}=200 \mathrm{~nm}$ and $w=50 \mathrm{~nm}$, (c) Gold nanowire with $\Lambda_{\mathrm{g}}=250 \mathrm{~nm}$ and $w=50 \mathrm{~nm}$, (d) Silver nanowire with $\Lambda_{\mathrm{g}}=200 \mathrm{~nm}$ and $d_{\mathrm{g}}=20 \mathrm{~nm}$, and $\Lambda_{\mathrm{g}}=250 \mathrm{~nm}$ and $d_{\mathrm{g}}=15 \mathrm{~nm}$ with template width $w=50 \mathrm{~nm}$.

The gold nanowire array surface with $\Lambda_{\mathrm{g}}=200 \mathrm{~nm}$ and $d_{\mathrm{g}}=20 \mathrm{~nm}$ (Fig. 4.6(b)) shows two resonances and the origin of the higher energy resonance is not known and further investigation is required. Figure 4.6(d) shows that the localized surface plasmon resonance wavelength can be tuned to $532 \mathrm{~nm}$ and $633 \mathrm{~nm}$ for a fixed silver thickness $t_{\mathrm{z}} \mathrm{nm}$ and different 
pitch $\Lambda_{\mathrm{g}}$ and nanogap spacing $d_{\mathrm{g}}$. In all cases, $\lambda_{\mathrm{L}}$ red-shifts when $d_{\mathrm{g}}$ is decreased, hence demonstrating a strong dependence on the couple-resonance mode in the nanogap.

The localized surface plasmon resonance can be assessed with the resonance quality factor $Q$, which is a dimensionless metric representing the ratio of energy stored in the resonator to energy loss per cycle. The experimental quality factor can be estimated with $Q_{\mathrm{L}} \approx \lambda_{\mathrm{L}} / \Delta \lambda$, where $\Delta \lambda$ is the full-width half-maximum of the reflectance spectrum and $\lambda_{\mathrm{L}}$ is the wavelength at the minimum reflectance $R_{\min }$. The localized surface plasmon resonance mode shape is modeled with a Lorentzian function and the corresponding $\Delta \lambda$ is determined. The quality factor of the gold nanowire array with $\Lambda_{\mathrm{g}}=250 \mathrm{~nm}$ and $d_{\mathrm{g}}=20 \mathrm{~nm}$ is $Q_{\mathrm{L}} \sim 10$ (Fig. 4.6(c)), and for the silver nanowire array with $\Lambda_{\mathrm{g}}=200 \mathrm{~nm}$ and $d_{\mathrm{g}}=20 \mathrm{~nm} Q_{\mathrm{L}} \sim 15$ (Fig. $4.6(\mathrm{~d})$ ).
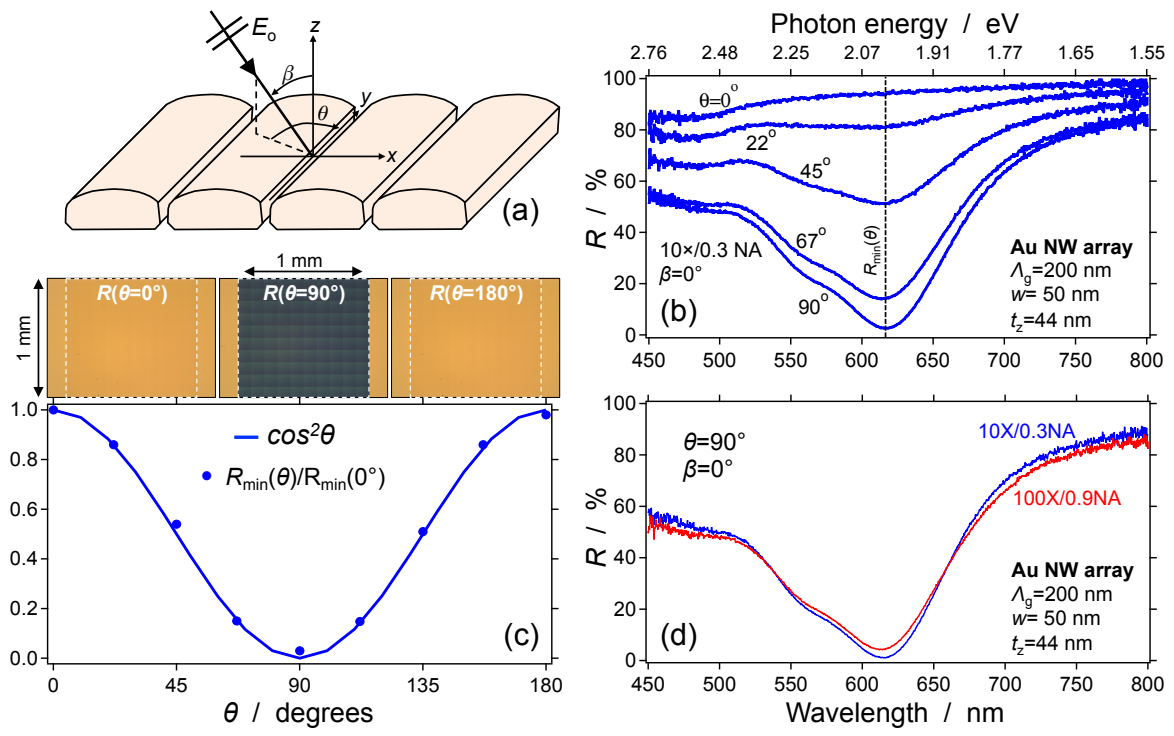

Figure 4.7. Measured local surface plasmon resonance dependence on the excitation polarization alignment to nanogap. (a) Reflectance measurement configuration, (b) Reflectance as the azimuthal angle of the p-polarized excitation source is varied from $\theta=0^{\circ}$ (no coupling) to $\theta=90^{\circ}$ (coupled), (c) Measured normalized reflectance minimum $\mathrm{R}_{\min }(\theta) / \mathrm{R}_{\min }\left(0^{\circ}\right)$ (solid dots) and $\cos ^{2}(\theta)$ (solid line) demonstrating the plasmonic coupling dependence. Upper inset: Reflectance images of gold nanowire surface (outlined in white dashed line) for different polarization alignments $\theta=0^{\circ}$ (no coupling), $\theta=90^{\circ}$ (coupled with dark blue reflected color), and $\theta=180^{\circ}$ (no coupling), (d) Measured reflectance of gold nanowire array with high magnification objective $(100 \times / 0.9 \mathrm{NA})$ and low magnification objective $(10 \times / 0.3 \mathrm{NA})$.

The relatively low experimental quality factors are typical for noble metals, such as gold and silver, due to absorptive damping in the metal [44], however, some inhomogeneous broadening of the resonance dip occurs to the finite surface roughness of the polycrystalline metal layer.

The localized surface plasmon resonance coupling is dependent on the alignment of the polarization of the normally incident $\left(\beta=0^{\circ}\right)$ excitation source to the gold nanowire nanogap as shown in reflectance measurements in Figure 4.7. The polarization of the white light 
excitation is fixed and the sample is rotated from $\theta=0^{\circ}$, where the source excitation polarization is parallel to the length of the nanowire (s-polarized) to $\theta=90^{\circ}$, where the electric field polarization of the source excitation is perpendicular to the length of the nanowire array (p-polarized) (Fig. 4.7(a)). The localized surface plasmon resonance coupling dependence on the excitation polarization is clearly shown as the magnitude of the reflectance dip decreases as excitation is rotated from p-polarization to s-polarization where no plasmonic coupling occurs and the reflectance of the subwavelength surface is similar to a planar (as-deposited) gold surface. As is well known from electromagnetic theory, the total electric field outside of the metal-nanowire is $\boldsymbol{E}_{\mathrm{s}}=\boldsymbol{E}+\boldsymbol{E}_{\mathrm{i}}$, where $\boldsymbol{E}$ is the incident electric field and $\boldsymbol{E}_{\mathrm{i}}$ is the induced or scattered electric field $[45,46]$.

Similar to the scattering theory of metal nanoparticles $\left|\boldsymbol{E}_{\mathrm{s}}\right|^{2}=I_{\mathrm{sp}} \sin ^{2} \theta$, where $I_{\mathrm{sp}}$ is the total intensity perpendicular to the plane of incidence, which is dependent on the excitation polarization alignment to the nanostructure, and therefore, the reflectance of the metalnanowire arrays is modulated according to the alignment of the excitation polarization to the nanogaps as a function of azimuthal angle $\theta$ (Fig. 4.7(a)). The polarization dependent reflectance from a gold nanowire array surface (Fig. 4.7(b)) shows that for p-polarized excitation aligned perpendicular to the nanogap $\left(\theta=90^{\circ}\right)$ results in the strongest plasmonic coupling of the incident radiation in the nanogap and subsequent reflectance $\operatorname{dip} R_{\min }$ at the coupling wavelength $\lambda_{\mathrm{L}}$, which corresponds to the maximum $\left|\boldsymbol{E}_{\mathrm{S}}\right|^{2}$ and $\left|\boldsymbol{E}_{\mathrm{c}}\right|^{2}$. As the alignment angle is rotated to $\theta=0^{\circ}$, where the p-polarized incident radiation is aligned parallel to the nanogap, no plasmonic coupling in the nanogap occurs, i.e. minimum $\left|\boldsymbol{E}_{\mathrm{s}}\right|^{2}$, and the subwavelength pitch gold nanowire array surface exhibits a reflectance similar to the planar gold surface. Consequently, the minimum reflectance is dependent on the alignment angle according to $R_{\min }(\theta) \propto \cos ^{2} \theta$, as shown in Fig. 4.7(c). The inset images of the large area (1 $\mathrm{mm}^{2}$ ) gold nanowire array surface show the plasmonic coupling of the p-polarized incident radiation is aligned parallel $\left(\theta=0^{\circ}\right.$ and $\left.\theta=180^{\circ}\right)$ to the length of the nanowires, where no plasmonic coupling occurs and the reflected image color in the patterned region (within the white dashed lines) is similar to the unpatterned gold layer (outside the white dashed lines), and for the p-polarized incident radiation aligned perpendicular $\left(\theta=90^{\circ}\right)$, where the strong plasmonic coupling is evident by the reflected color change to dark blue in the patterned regions (Fig. 4.7(c)).

The dark blue reflected color corresponds to a yellow complementary color, which corresponds to a $\lambda_{\mathrm{L}} \approx 633 \mathrm{~nm}$ from $R_{\min }\left(\theta=90^{\circ}\right)$, as shown in Fig. 4.7(b) [47]. The uniformity of the gold nanowire array surface with $\Lambda_{\mathrm{g}}=200 \mathrm{~nm}$ and $d_{\mathrm{g}}=20 \mathrm{~nm}$ is demonstrated using 
reflectance spectroscopy, as shown in Fig. 4.7(d), where the reflection spectrum collected using a low magnification microscope objective $(10 \times / 0.3 \mathrm{NA})$ with a large spot diameter is shown with the reflection spectra collected with a high magnification microscope objective $(100 \times / 0.9$ NA) with a much smaller spot diameter. The large area reflectance spectra are nearly identical to the small area reflectance measurements, thus demonstrating the high degree of uniformity in the pitch and nanogap dimensions of the gold nanowire array surfaces.

\subsection{Surface-enhanced Raman spectroscopy}

The gold nanowire arrays are applied as SERS substrates by covering the gold nanowire array surface with a monolayer of benzenethiol (BT) molecules, which provides an estimation of the uniformity of the spatially averaged enhancement factor from a small number of molecules. BT is a commonly used SERS probe molecule since it has a small number of wellcharacterized and strong Raman active modes, and does not fluoresce in the visible spectrum. As described in Chapter 2, the measured Raman intensity [units: of photons $s^{-l}$ ] of a particular vibration band can be estimated with

$$
\phi_{S}=\left(\lambda_{0} / h c\right) I_{0} n_{d}\left(d \sigma_{s} / d \Omega\right) N_{S E R S} A_{s}^{-1} \kappa\left\langle G^{E M}\right\rangle,
$$

where $\left\langle G^{\mathrm{EM}}\right\rangle$ is an ensemble average enhancement factor from the $N_{\text {SERS }}$ molecules in the collection volume at each measurement location. In Figure 4.8(a), a representative SERS spectrum of a BT chemisorbed monolayer on a gold nanowire array surface is shown with the dominant Raman active vibrational modes: $416 \mathrm{~cm}^{-1}, 691 \mathrm{~cm}^{-1}, 994 \mathrm{~cm}^{-1}, 1018 \mathrm{~cm}^{-1}, 1068$ $\mathrm{cm}^{-1}$ and $1565 \mathrm{~cm}^{-1}$, all of which have been previously reported. The $416 \mathrm{~cm}^{-1}, 691 \mathrm{~cm}^{-1}$ and $1068 \mathrm{~cm}^{-1}$ bands are a combination of the ring and C-S stretch modes, the $994 \mathrm{~cm}^{-1}$ band is a ring mode, the $1018 \mathrm{~cm}^{-1}$ band from an $\mathrm{H}$-stretch, and the $1565 \mathrm{~cm}^{-1}$ band is a $\mathrm{C}-\mathrm{C}$ ring stretch mode [49]. Note that the absence of the $917 \mathrm{~cm}^{-1}$ vibration mode, which is present in the normal Raman BT spectrum, in the SERS spectrum is a good indicator of BT monolayer formation. The measured spectrum of BT on a planar gold layer is also shown in Fig. 4.8(a), which does not contain any detectable vibration bands and establishes the background noise floor of the measurement system. Note that the broad background signal is not present in the BT chemisorbed to the planar gold surface (Fig. 4.8(a)), or the neat BT solution measurements. Each vibration band of a measured SERS spectrum at each measurement location is modeled with a Lorentzian function and the background signal is modeled with a polynomial function. The integrated intensity of each vibration band $K^{v}$ was calculated by integrating the area of the fitted Lorentzian function after the removal of the background. The 
fitting accuracy of the Lorentzian function was comparable to the Voigt functions and is used for modeling all measured vibrational spectra. An example of a modeled spectrum is shown in the upper plot (blue solid line) of Fig. 4.8(a). The average nanogap spacing $d_{\mathrm{g}}=(22 \pm 2) \mathrm{nm}$, which was estimated from the 10 nanogaps (black region) is shown in Fig. 4.4(a). Figure 4.8(c) shows a Raman image of the integrated intensity of the combined ring-bending and C-S stretch modes $\left(1068 \mathrm{~cm}^{-1}\right.$ band) from 2500 full spectra measured over an area of $1600 \mu \mathrm{m}^{2}$, which demonstrates the high spatial uniformity of the nanogap local surface plasmon resonance of the gold nanowire array substrates, where $100 \%$ of the measured locations generate very large Raman intensities.
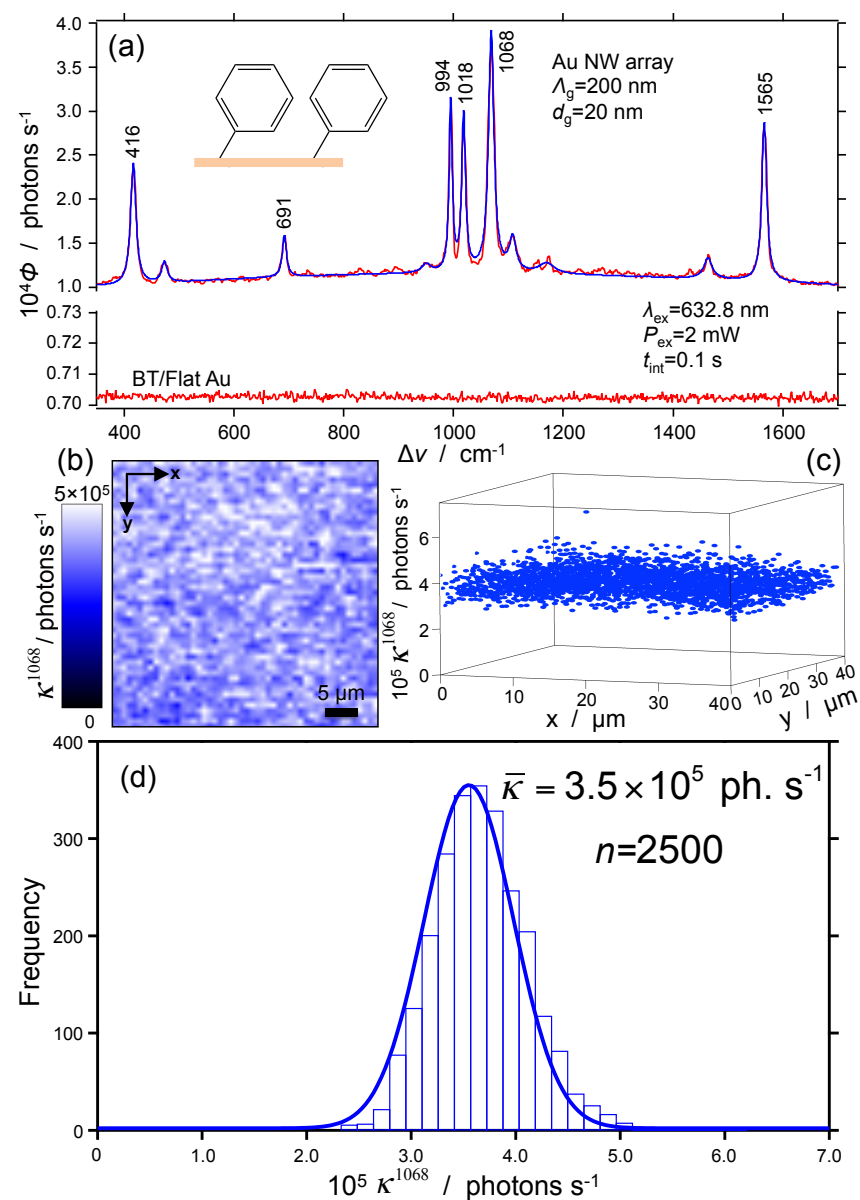

Figure 4.8. Measured SERS spectra of BT chemisorbed on gold nanowire array surface. (a) Top: Measured (red) spectrum from surface with pitch $\Lambda_{\mathrm{g}}=200 \mathrm{~nm}$ and $d_{\mathrm{g}}=20 \mathrm{~nm}$ (measured with SEM), and modeled Lorentzian bands (blue); Bottom: Measured spectrum from BT chemisorbed on a planar (as-deposited) gold layer, (b) Raman image of the integrated intensity of the $1068 \mathrm{~cm}^{-1}$ band over a $40 \times 40 \mu \mathrm{m}^{2}$ area, (c) Three-dimensional scatter plot of the $1068 \mathrm{~cm}^{-1}$ band integrated intensity, (d) Histogram of the $1068 \mathrm{~cm}^{-1}$ band integrated intensity from Raman image and modeled Gaussian distribution.

The integrated intensity of the $1068 \mathrm{~cm}^{-1}$ vibration band is shown in the spatial Raman integrated intensity image map (Fig. 4.8(c)) and the three-dimensional scatter-plot shown in Fig. 4.8(d). The histogram of the measured integrated intensity and fitted Gaussian 
distribution with mean $\bar{K}^{1068} \approx 3.5 \times 10^{5}$ photon $\mathrm{s}^{-1}$ with standard deviation $S_{\bar{K}}^{1068} \approx 0.4 \times 10^{5}$ photon $\mathrm{s}^{-1}$, shown in Figure 4.9(e). From the scatter and histogram plots, the measured SERS spectra is highly uniform with every measurement location providing a large enhancement factor with about $11 \%$ variation across the measured surface, which is attributed to the new fabrication technique with controllable and uniform nanogaps with extremely large nanogap length density $\left(\sim 1 \mathrm{~km} \mathrm{~cm}^{-2}\right)$ that provides a large number of scattering hotspots at each measurement location. Additionally, the sample-to-sample variation has been estimated at about $10 \%$.
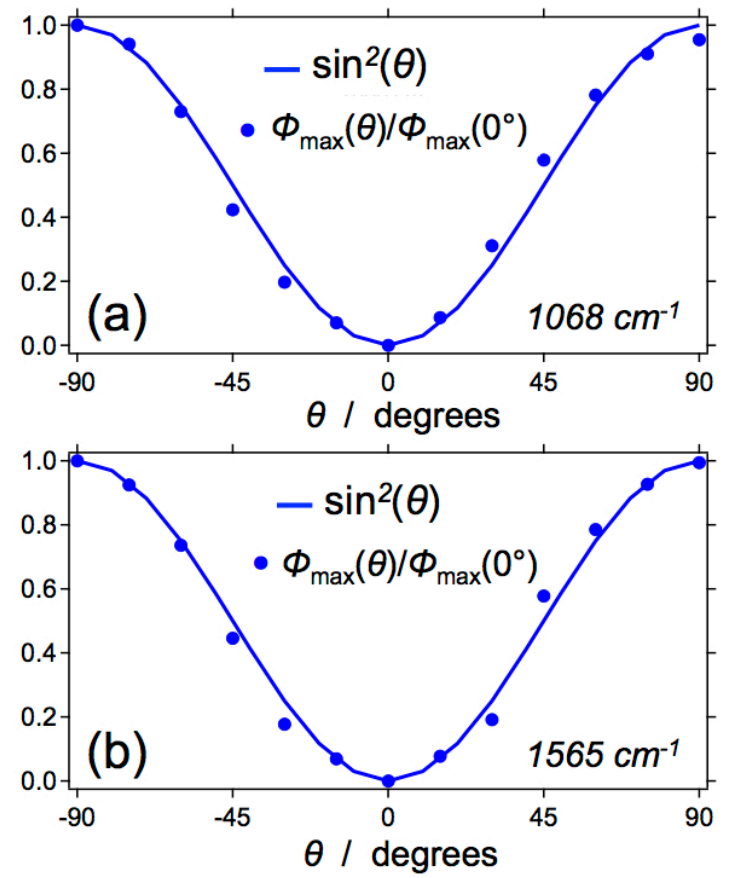

Figure 4.9. Normalized polarization dependent Raman intensity measurements of chemisorbed BT on gold nanowire array surfaces with pitch $\Lambda_{\mathrm{g}}=200 \mathrm{~nm}$ and $d_{\mathrm{g}}=20 \mathrm{~nm}$ (measured). (a) $1068 \mathrm{~cm}^{-1}$ band, (b) $1565 \mathrm{~cm}^{-1}$ band.

The analytical enhancement factor is commonly used to assess the magnitude of the Raman enhancement $G_{\mathrm{i}} \approx\left(K_{\mathrm{i}} / N_{\mathrm{SERS}}\right)\left(K_{\mathrm{NR}} / N_{\mathrm{NR}}\right)^{-1}$, where $K_{\mathrm{i}}$ and $K_{\mathrm{NR}}$ and are the integrated intensities from the SERS and conventional Raman measurements, respectively, and $N_{\text {SERS }}$ and $N_{\mathrm{NR}}$ are the number of molecules in the collection volume of the SERS and conventional measurements, respectively. Furthermore, the spatially averaged SERS enhancement of the $1068 \mathrm{~cm}^{-1}$ band estimated from 2500 spatial Raman data (Figs. 4.8(c) and 4.8(d)), and conventional Raman measurements of neat BT, is $\bar{G} \approx 1.2 \times 10^{7}$, which is extremely large considering that all of the BT molecules chemisorbed to the gold nanowire surface have been included in the estimation of the enhancement factor, while it is well known that the molecules in the nanogap region generate the majority of the measured signal. The standard deviation of the spatially averaged SERS enhancement factor is $0.2 \times 10^{7}$, which represents an $11 \%$ enhancement factor variation averaged over the 2500 measurements. The large spatially 
averaged SERS enhancement and small variance (Fig. 4.8(d)) is very significant compared to previously reported distributions [50], where a significant number of measurement locations were not enhanced.

As previously described, the generation of the localized surface plasmon resonance is dependent on the alignment of the excitation polarization with the nanogaps, and since the magnitude of the electromagnetic field in the nanogap $\left|\boldsymbol{E}_{\mathrm{c}}\right|$ is related to the SERS enhancement as $G_{\mathrm{i}} \propto\left|M_{\mathrm{c}}\right|^{2}$, then optimizing the polarization alignment to the nanogap also maximizes the SERS signal. For most plasmonic substrates, aligning the excitation polarization with the geometry of the nanostructures is very important to maximize the electromagnetic enhancement. The polarization alignment was performed by manual rotation of the excitation laser beam using a $\lambda / 2$ rotator plate. From the polarization dependent reflectance measurements (Figs. 4.8(b) and 4.8(c)), the minimum reflectance was shown to follow $R_{\mathrm{m}}(\theta) \propto \cos ^{2} \theta$, hence, the Raman enhancement is $G_{\mathrm{i}} \propto \sin ^{2} \theta$, and therefore, $\phi_{\max }(\theta) \propto \sin ^{2} \theta$, where the maximum Raman enhancement occurs at $\theta=90^{\circ}$, shown in Fig. 4.9.

\subsection{Conclusions}

The optical characterization of the metal nanowire array surfaces using reflection spectroscopy and surface-enhanced Raman spectroscopy has been presented. Reflection spectroscopy is used to demonstrate the plasmonic functionality, and tunability and uniformity of the dimensions of the metal nanowire array surfaces, pitch and nanogap spacing, with a normally incident linearly polarized white light source that is aligned perpendicular to the nanogaps. Surface-enhanced Raman spectroscopy is used to assess the uniformity of the electromagnetic enhancement of the metal nanowire arrays surfaces by covering the array surfaces with a benzenethiol self-assembled monolayer, which provides an estimation of the uniformity of the spatially averaged enhancement factor from a small number of molecules. Finally, the surface selection rules of benzenethiol self-assembled monolayers on silver nanowire array surfaces have been evaluated and it has been shown that $a_{1}$ Raman vibration modes dominate the spectra.

\section{References}

[1] S.A. Maier, M. L. Brongersma, P.G. Kik, S. Meltzer, A.A.G. Requicha, and H. A. Atwater, Adv. Mater. Weinheim, 13, 1501, 2001.

[2] D. Pines and D. Bohm "A collective description of electron interactions: II. Collective vs individual particle aspects of the interactions," Phys. Rev. 85, 338, 1952.

[3] R.H. Ritchie, "Plasma losses by fast electrons in thin films," Phys. Rev. 106, 874, 1957.

[4] M. Fleischmann, P.J. Hendra, and A.J. McQuillan, "Raman spectra of pyridine at a silver electrode," Chem. Phys. Lett. 26, 163, 1974. 
[5] M. Moskovits, "Surface roughness and the enhanced intensity of Raman scattering by molecules adsorbed on metals," J. Chem. Phys. 69, 4159, 1978.

[6] C.K. Chen, A.R.B. de Castro, and Y.R. Shen "Surface-enhanced second-harmonic generation," Phys. Rev. Lett. 46, 145, 1981.

[7] J. Homola, S.S. Yee, and G. Gauglitz, "Surface plasmon resonance sensors: review" Sens and Actuat. B 54, 3, 1999.

[8] S. Nie and S.R. Emory, "Probing single molecules and single nanoparticles by surfaceenhanced Raman scattering," Science 275, 1102, 1997.

[9] K. Kneipp, Y. Wang, H. Kneipp, L. T. Perelman, I. Itzkan, R.R. Dasari, and M.S. Feld, "Single molecule detection using surface-enhanced Raman scattering (SERS)," Phys. Rev. Lett. 78, 1667, 1997.

[10] D. Pines "Collective energy losses in solids," Rev. Mod. Phys. 28, 184, 1956.

[11] L. Tonks and I. Langmuir "Oscillations in ionized gases," Phys. Rev. 33, 195, 1929.

[12] C. Kittel, Introduction to Solid State Physics. $8^{\text {th }}$ Ed. John Wiley \& Sons, 2005.

[13] S.A. Maier, Plasmonics: Fundamentals and Applications. Springer, 2007.

[14] U. Kreibig and M. Vollmer, Optical Properties of Metal Clusters. Springer-Verlag, 1995.

[15] C.J. Powell and J.B. Swan "Origin of the characteristic electron energy losses in aluminum," Phys. Rev. 115, 869, 1959.

[16] C.J. Powell and J.B. Swan "Origin of the characteristic electron energy losses in magnesium," Phys. Rev. 116, 81, 1959.

[17] E.A. Stern and R.A. Ferrell "Surface plasmon oscillations of a degenerate electron gas," Phys. Rev. 120, 130, 1960.

[18] H. Raether, Surface Plasmons on Smooth and Rough Surfaces and on Gratings. Springer-Verlag, 1986.

[19] M. Guerrisi, R. Rosei, and P. Winsemius, "Splitting of the interband absorption edge in Au," Phys. Rev. B 12, 557-563, 1975.

[20] P.B. Johnson and R.W. Christy, "Optical constants of the noble metals." Phys. Rev. B 6, 4370-4379, 1972.

[21] G.T. Boyd, Z.H. Yu, and Y.R. Shen, "Photoinduced luminescence from the noble metals and its enhancement on rough surfaces," Phys. Rev. B 33, 7923-7936, 1986.

[22] N.A. Abu Hatab, J.M. Oran, and M.J. Sepaniak, "Surface-enhanced Raman spectroscopy substrates created via electron beam lithography and nanotransfer printing," ACS Nano 2, 377-385, 2008.

[23] A.G. Brolo, E. Arctander, R. Gordon, B. Leathem, and K.L. Kavanagh, "Nanoholeenhanced Raman scattering," Nano Lett. 4, 2015-2018, 2004.

[24] D.P. Fromm, A. Sundaramurthy, P.J. Schuck, G. Kino, and W.E. Moerner, "Gapdependent optical coupling of single "bowtie" nanoantennas resonant in the visible," Nano Lett. 4, 957-961, 2004.

[25] J.C. Hulteen and R.P. Van Duyne. "Nanosphere lithography: A materials general fabrication process for periodic particle array surfaces," J. Vacuum Sci. Technol. A 13, 1553-1558, 1995.

[26] L. Rodríguez-Lorenzo, R.A. Alvarez Puebla, I. Pastoriza-Santos, S. Mazzucco, O. Stéphan, M. Kociak, L.M. Liz-Marzán, and F.J. García de Abajo, "Zeptomole detection through controlled ultrasensitive surface-enhanced Raman scattering," J. Am. Chem. Soc. 131, 4616-4618, 2009.

[27] S. Shanmukh, L. Jones, J. Driskell, Y. Zhao, R. Dluhy, and R.A. Tripp, "Rapid and sensitive detection of respiratory virus molecular signatures using a silver nanorod array SERS substrate," Nano Lett. 6, 2630-2636, 2006.

[28] S.C. Kitson and J.R. Sambles, "Full photonic band gap for surface modes in the visible," Phys. Rev. Lett. 77, 2670-2673, 1996. 
[29] L. Gunnarsson, E.J. Bjerneld, H. Xu, S. Petronis, B. Kasemo, and M. Käll, "Interparticle coupling effects in nanofabricated substrates for surface-enhanced Raman scattering," Appl. Phys. Lett. 78, 802-804, 2001.

[30] J.A. Porto, F.J. Garcia-Vidal, and J.B. Pendry, "Transmission resonances on metallic gratings with very narrow slits," Phys. Rev. Lett. 83, 2845-2848, 1999.

[31] N. Perney, J. Baumberg, M. Zoorob, M. Charlton, S. Mahnkopf, and C.M. Netti, "Tuning localized plasmons in nanostructured substrates for surface-enhanced Raman scattering," Opt. Express 14, 847-857, 2006.

[32] H. Gao, J. Henzie, M.H. Lee, and T.W. Odom, "Screening plasmonic materials using pyramidal gratings," Proc. Natl. Acad. Sci. USA 105, 20146-20151, 2008.

[33] T.-H. Lin, N.C. Linn, L. Tarajano, B. Jiang, and P. Jiang, "Electrochemical SERS at periodic metallic nanopyramid arrays," J. Phys. Chem. C 113, 1367-1372, 2009.

[34] H.-Y. Wu, C. J. Choi, and B.T. Cunningham, "Plasmonic nano-gap-enhanced Raman scattering using a resonant nanodome array," Small 8, 2878-2885, 2012.

[35] L. Le Thi Ngoc, M. Jin, J. Wiedemair, A. van den Berg, and E.T. Carlen, "Large area metal nanowire arrays with tunable sub-20 nm nanogaps," ACS Nano 7, 5223-5234, 2013.

[36] J. Wiedemair, L. Le Thi Ngoc, A. van den Berg, and E.T. Carlen, "Surface-enhanced Raman spectroscopy of self-assembled monolayer conformation and spatial uniformity on silver surfaces," J. Phys. Chem. C 118, 11857-11868, 2014.

[37] T. Yuan, L. Le Thi Ngoc, J. van Nieuwkasteele, M. Odijk, A. van den Berg, H. Permentier, R. Bischoff, and E.T. Carlen, "In situ surface-enhanced Raman spectroelectrochemical analysis system with a hemin modified nanostructured gold surface," Anal. Chem. February 2015.

[38] N.J. Halas, S. Lal, W.-S. Chang, S. Link, and P. Nordlander, "Plasmons in strongly coupled nanostructures," Chem. Rev. 111, 3913-3961, 2011.

[39] F.J. Garcia-Vidal and J.B. Pendry "Collective theory for surface enhanced Raman scattering," Phys. Rev. Lett. 77, 1163-1166, 1996.

[40] M. Moskovits, "Surface-enhanced spectroscopy," Rev. Mod. Phys. 57, 783-826, 1985.

[41] T.D. Corrigan, S. Guo, R.J. Phaneuf, and H.J. Szmacinski "Enhanced fluorescence from periodic arrays of silver nanoparticles," Fluoresc. 15, 777-784, 2005.

[42] S.R. Stuart and D.G. Hall "Absorption enhancement in silicon-on-insulator waveguides using metal island films," Appl. Phys. Lett. 73, 3815-3817, 1998.

[43] R. Zia, J.A. Schuller, A. Chandran, and M.L. Brongersma, "Plasmonics: The wave of chip-scale device technologies," Mater. Today 9, 20-27, 2006.

[44] M.D. Arnold and M.G. Blaber, "Optical performance and metallic absorption in nanoplasmonic systems," Opt. Express 17, 3835-3847, 2009.

[45] M. Born and E. Wolf, Principles of Optics. 7th Ed., Cambridge University Press, 1999.

[46] H. Xu, J. Aizpurua, M. Käll, and P. Apell "Electromagnetic contributions to singlemolecule sensitivity in surface-enhanced Raman scattering," Phys. Rev. E. 62, 43184324, 2000.

[47] T. Owen, Fundamentals of Modern UV-Visible Spectroscopy: Primer; Agilent Technologies: Santa Clara, CA, 2000; Pub. 5980-1397E.

[48] W.E. Moerner, "Examining nanoenvironments in solids on the scale of a single, isolated impurity molecule," Science 265, 46-53, 1994.

[49] T.H. Joo, M.S. Kim, and K. Kim "Surface-enhanced Raman scattering of benzenethiol in silver sol," J. Raman Spectrosc. 18, 57-60, 1987.

[50] M. Jin, H. van Wolferen, H. Wormeester, A. van den Berg, and E.T. Carlen "Large-area nanogap plasmon resonator arrays for plasmonics applications," Nanoscale 4, 47124718, 2012. 



\section{Chapter 5}

\section{Plasmon-modulated photoluminescence ${ }^{1}$}

An overview of conventional photoluminescence from polycrystalline gold layers and plasmon-modulated photoluminescence from gold nanostructures is first presented in this chapter, and is followed by a systematic study of plasmonmodulated photoluminescence from gold nanowire array surfaces presented in Chapter 3. The study outlines the importance of the spectral overlap of the excitation and surface plasmon resonance (of the gold nanowire array surface) energies with the interband transition points of the electronic band structure of gold. In addition, it is shown that the plasmon-modulated photoluminescence is a strong source of the background continuum observed in surface-enhanced Raman scattering spectra from surface adsorbed molecules on gold nanostructures when a red excitation laser is used.

\footnotetext{
${ }^{1}$ Adapted from: L. Le Thi Ngoc, J. Wiedemair, A. van den Berg, and E.T. Carlen, "Plasmon-modulated photoluminescence from gold nanostructures and its dependence on plasmon resonance, excitation energy, and band structure," Opt. Express 23, 5547, 2015.
} 


\subsection{Introduction to plasmon-enhanced photoluminescence}

Photoluminescence from thin copper and gold films was first reported forty-five years ago, and was attributed to the direct recombination of photoexcited $s p$-conduction band electrons with $d$-band holes that were scattered to momentum states less than Fermi momentum [1]. Many years later the discoveries of surface-enhanced Raman scattering (SERS) and second harmonic generation (SHG) on roughened noble metal surfaces stimulated further investigation into single-photon and multi-photon metal photoluminescence [2-5]. Boyd and co-workers conducted a series of single-photon and multiphoton experiments on smooth and roughened surfaces of silver, copper, and gold [5], where photoluminescence peaks were assigned interband transitions near the $X$ - and $L$-symmetry points in the electronic band structure. Furthermore, they reported that roughened surfaces increase the photoluminescence absorption and emission efficiencies through the enhancement of local optical fields by a localized surface plasmon resonance. The photoluminescence process in noble metals has been described as a three-step process consisting of $d$-hole: i) Photoexcitation (quantum efficiency, $q_{\mathrm{E}}$ ), ii) Relaxation (quantum efficiency, $q_{\mathrm{R}}$ ), and iii) Recombination (quantum efficiency, $\left.q_{\mathrm{D}}\right)$ due to interband transitions near the $X$ - and $L$-symmetry points $[5,6]$. In the first step, electrons are induced into the $s p$-band by incident radiation, and holes into the $d$ band, thus establishing non-equilibrium populations of electrons and holes. The photoexcited populations are subsequently subjected to a thermalization process consisting of electronelectron and electron-phonon scattering processes, which results in very fast energy and momentum relaxation. In the final step, a small fraction of $d$-band holes, with momentum less than the Fermi momentum, recombine radiatively with $s p$-band electrons via vertical momentum space transitions $[5,6]$. The total quantum efficiency of the photoluminescence process can be expressed as a combination of the quantum efficiency of each process, $Q_{\mathrm{E}}=q_{\mathrm{E}} q_{\mathrm{R}} q_{\mathrm{D}}$ [5-7]. In conventional metal photoluminescence, $q_{\mathrm{R}}$ and $q_{\mathrm{D}}$ are dominated by fast non-radiative relaxation processes, thus resulting in very low $Q_{\mathrm{E}}[5,7]$. In the visible spectrum, gold photoluminescence originates from $5 d-6 s p$ interband transitions near the $X$ and $L$-symmetry points in the electronic band structure, which significantly impacts photoluminescence especially when modulated by surface plasmon resonances in nanostructures. Therefore, some important details of the electronic band structure of gold that are relevant for understanding plasmon-enhanced photoluminescence are first discussed. It should be noted that photoluminescence due to intraband transitions is also possible, which involves electron-hole pairs generated in the $s p$-band by near-infrared excitation energies, but is typically much weaker than photoluminescence due to interband transitions [8]. 
The motivation for studying plasmon-enhanced photoluminescence stems from an interest in developing nanostructured noble metal surfaces for SERS [9,10], and their application to surface-enhanced Raman spectroscopy [11] and spectroelectrochemistry [9], where all SERS spectra are accompanied by a strong background continuum. The background continuum that accompanies the SERS spectra from molecules adsorbed to metal surfaces has been studied extensively since the discovery of SERS more than forty years ago, and there have been many explanations of the observed phenomena, which have been previously reviewed $[4,12,13]$. It is important to note that many of the early studies were conducted on silver surfaces using a variety of conditions and adsorbed molecules, and the background continuum accompanying SERS spectra from silver nanostructures is very different compared to the continuum generated on gold nanostructures. Currently, the most commonly accepted models of the background continuum generation mechanism involve the electronic interaction of the surface adsorbed molecules and the free electrons in the metal layer near the interface [14-17]. However, it has been acknowledged that plasmon-enhanced photoluminescence from the rough noble-metal films is closely related to SERS and may contribute to the background continuum [4,18]. Other models based on inelastic scattering, adsorbed molecule luminescence, and resonant light scattering have been proposed [14,18-20].

\subsection{Optical and electronic properties of gold}

Gold has a face centered cubic crystal structure with a distorted spherical free electron Fermi surface that touches the first Brillouin zone on the hexagonal faces of the crystal, thus producing a neck region, called the $L$-symmetry point, and belly region, called the $X$ symmetry point [21]. In the visible spectrum, gold photoluminescence primarily originates from $5 d-6 s p$ interband transitions near the $X$ - and $L$-symmetry points in the electronic band structure. The energy band (E-k) diagrams of the partial electronic band structure of gold in the first Brillouin zone are shown in Figs. 5.1(a) and 5.1(b). The interband transitions $\Delta_{\mathrm{X}}$ and $\Delta_{\mathrm{L}}$ are shown near the $X$ - and $L$-symmetry points, respectively, where the $5 d$ bands are relatively flat. A critical point is defined as the energy where $\mathrm{d} E(k) / \mathrm{d} k=0$ of two bands, hence are parallel, which corresponds to a van Hove singularity in the joint density of electron states. When the bands are not parallel, i.e. $\mathrm{d} E(k) / \mathrm{d} k \neq 0$, the joint density of states will be reduced, however, the absorption rate due to interband transitions can be significant, especially when one of the bands is relatively flat, as is the case of the $5 d$ bands near the $X$ and $L$-symmetry points (Fig. 5.1). It should be noted that photoluminescence due to intraband transitions is also possible, which involves electron-hole pairs generated in the $s p$-band by near-infrared excitation energies, but is typically weaker than photoluminescence due to 
interband transitions [8]. Beaglehole developed an energy band structure of gold and identified a high density of states in the first Brillouin zone near the $X$ - and $L$-symmetry points, where the $d$-bands are relatively flat in momentum space $[22,23]$.
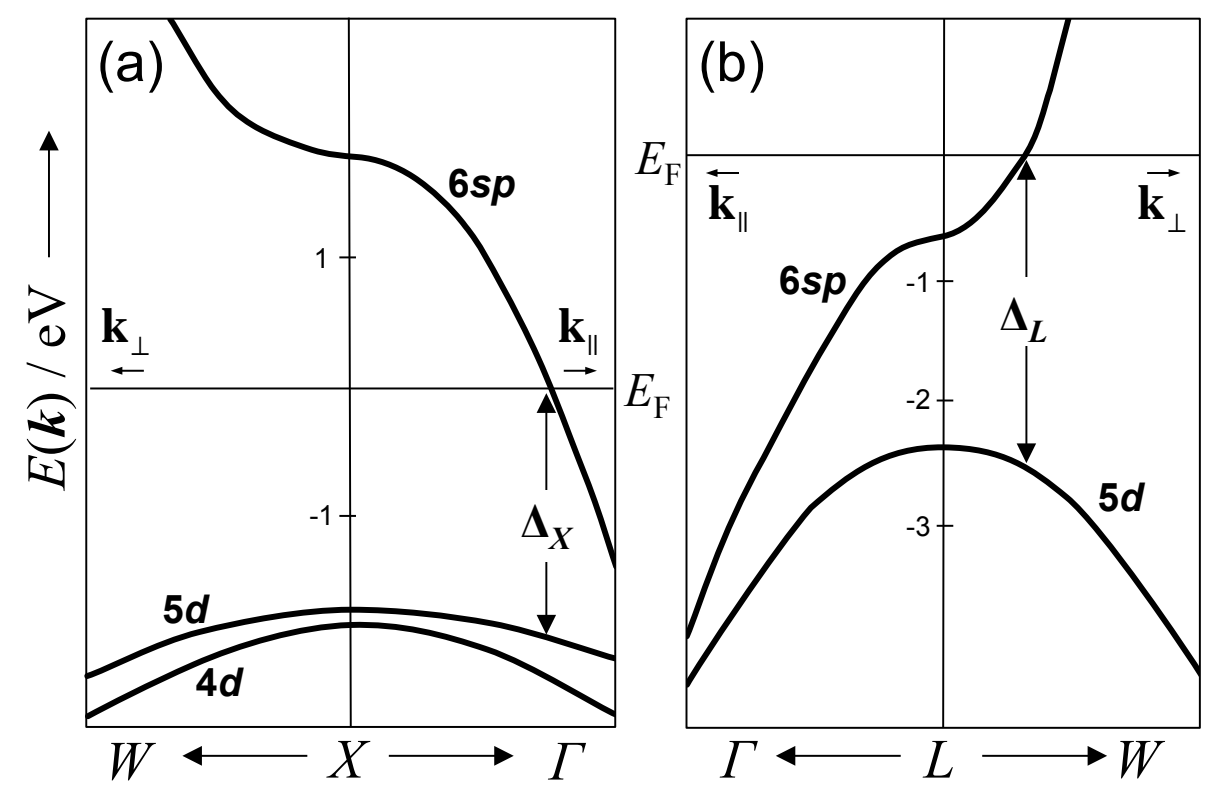

Figure 5.1. Select symmetry regions of the first Brillouin zone of the energy band $E(k)$ diagram of gold. (a) $X$-symmetry point and interband transition $\Delta_{\mathrm{X}}$, (b) $L$-symmetry point and interband transition $\Delta_{\mathrm{L}}$. The electron wave vector $\mathrm{k}_{\|}$points from the $X$ - and $L$-symmetry points to the $\Gamma$-symmetry point, and $\mathrm{k}_{\perp}$ point in the direction of the $W$-symmetry point [27].

It is now well established that the $d$-band density of states is larger and more strongly localized near the symmetry points compared to the $s p$-band, which have a more broad distribution of allowed energy states [24-27]. Although the selection rules for conventional dipole transitions between the $d$-band and $s p$-band states are forbidden, the small transition matrix elements, from Fermi's golden rule, are compensated by the large $d$-band density of electron states near the $X$ - and $L$-symmetry points. For plasmon-enhanced interband transitions, the conventional selection rules for dipole processes may not strictly hold due to the strongly confined fields with large field gradients [8]. Geurrisi and co-workers were the first to observe that the optical absorption of gold is split between two interband transitions, one located near the $X$-symmetry point with $\Delta_{\mathrm{X}} \approx 1.94 \mathrm{eV}$, and the other near the $L$-symmetry point with $\Delta_{\mathrm{L}} \approx 2.45 \mathrm{eV}$ [27]. The interband transition energy near the $X$-symmetry point was later reported to be $\Delta_{\mathrm{X}} \approx 1.84 \pm 0.02 \mathrm{eV}$, after correcting for intraband absorption [28,29]. In single-photon experiments, Boyd, et al. assigned photoluminescence peaks at $2.4 \mathrm{eV}$ to the $5 d-6 s p$ interband transition $\Delta_{\mathrm{L}}$, at $2.2 \mathrm{eV}$ to the combination of the $5 d-6 s p$ interband transition $\Delta_{\mathrm{L}}$ and the $4 d$-6sp interband transition near $\Delta_{\mathrm{X}}$, and at $1.9 \mathrm{eV}$ to the $5 d-6 s p$ interband transition $\Delta_{\mathrm{X}}$. The energy band $(E-k)$ diagrams of the partial electronic band structure of gold near the $X$ - and $L$-symmetry points in the first Brillouin zone are shown in Figs. 5.1(a) and 
5.1(b), respectively [27]. For the excitation energies considered in this article $\left(\hbar \omega_{01}=1.96 \mathrm{eV}\right.$ and $\hbar \omega_{02}=2.33 \mathrm{eV}$ ), which are commonly used for plasmonics applications, the $\Delta_{\mathrm{X}}$ and $\Delta_{\mathrm{L}}$ interband transitions of the $5 d$ and $6 s p$ bands are the most important for the optical response of gold in the visible spectrum $[5,29]$.

Despite extensive work to understand the electronic band structure of gold and the details of the interband absorption energies near the $X$ - and $L$-symmetry points [27], very often only the interband transition $\Delta_{\mathrm{L}}$ is referred to when describing the optical absorption of gold [3032]. This is reasonable in many cases considering the fact that the absorption cross section at $\Delta_{\mathrm{X}}$ is much weaker than at $\Delta_{\mathrm{L}}[5,27,33]$. However, when plasmon-enhanced photoluminescence is combined with moderately high power laser sources with emission energies located near $\Delta_{\mathrm{X}}$ and $\Delta_{\mathrm{L}}$, then both symmetry points must be considered [34]. Furthermore, for excitation energies in the near-infrared, intraband transitions can produce photoluminescence, which can also be plasmon-enhanced in gold nanostructures $[8,35]$.

The optical response of gold can be modeled with the real and imaginary parts of the dielectric function $\varepsilon(\omega)=\varepsilon_{1}(\omega)+i \varepsilon_{2}(\omega)$, which depend on the joint density of states for the bands involved in each interband transition. For excitation energies in the visible spectrum, the Lorentz-Drude model can describe the dielectric response of metals

$$
\varepsilon(\omega)=\varepsilon_{\infty}-\frac{\omega_{p}^{2}}{\omega^{2}-i \gamma \omega}+\sum_{i} \chi_{i}(\omega)
$$

where $\varepsilon_{\infty}$ is a constant term to account for the ion-core contribution at long wavelengths, $\omega_{p}^{2} /\left(\omega^{2}-i \gamma \omega\right)$ is the free electron contribution to the dielectric function, $\omega_{p}=\left(n q^{2} / \varepsilon_{0} m_{e}\right)^{1 / 2}$ is the plasma frequency, $\gamma$ is the electron damping rate, and $\chi(\omega)$ are the susceptibility terms that represent interband transitions in the joint density of states of the electronic band structure [30,31,36,37]. Despite reports of dielectric function models that accommodate the asymmetric band shapes with reasonably good fits over large frequency ranges, the extracted model parameters typically do not correspond to the important interband transitions in the energy band diagram [30,31,33]. Figure 5.2 shows measured $\varepsilon_{1}(\omega)$ and $\varepsilon_{2}(\omega)$ data from Thèye (solid red circles) [33], and Johnson and Christy (solid blue triangles) [38]. The solid black lines are generated from an analytical model [37] with interband transition energies $\Delta_{\mathrm{X}}=1.84 \mathrm{eV}$ and $\Delta_{\mathrm{L}}=2.45 \mathrm{eV}^{2}$. The gray shaded regions indicate the energy range between $\Delta_{\mathrm{X}}$ and $\Delta_{\mathrm{L}}$. The

\footnotetext{
${ }^{2}$ Susceptibility terms $\chi_{i}(\omega)=A_{i}\left[e^{i \theta}\left(\omega_{i}-\omega-i \gamma_{i}\right)^{-1}+e^{-\theta \theta}\left(\omega_{i}+\omega+i \gamma_{i}\right)^{-1}\right]$ fit to the measured data with $\varepsilon_{\infty}=1.5, \omega_{\mathrm{p}}=1.37 \times 10^{16} \mathrm{~s}^{-1}$, $\gamma_{\mathrm{p}}=0.14 \times 10^{15} \mathrm{~s}^{-1}, A_{1}=0.4 \times 10^{15}, \gamma_{1}=1.2 \times 10^{15} \mathrm{~s}^{-1}, \omega_{1}=\Delta_{\mathrm{X}} / \hbar, \theta=-\pi / 2.7, A_{2}=0.95 \times 10^{16}, \gamma_{2}=1.4 \times 10^{15} \mathrm{~s}^{-1}$, and $\omega_{2}=\Delta_{\mathrm{L}} / \hbar$.
} 
excitation energies $\hbar \omega_{01}$ and $\hbar \omega_{02}$ used in this article are shown with red and green dashed lines, respectively. It has been previously established that interband transitions near $\Delta_{\mathrm{X}}$ have broad absorption profiles, in contrast to the absorption profile near $\Delta_{\mathrm{L}}$, both of which are not clearly represented in the experimental data of gold $[33,38]$. The absorption does increase for photon energies above $\Delta_{\mathrm{L}}$. The absorption increase for photon energies below approximately $\hbar \omega \approx 1.5 \mathrm{eV}$ is due to the free-electron behavior of gold. When plasmon-modulated photoluminescence is combined with moderate-power laser sources with emission energies located between $\Delta_{\mathrm{X}}$ and $\Delta_{\mathrm{L}}$, the $X$-symmetry point dominates the photoluminescence response, which will be described in subsequent sections.

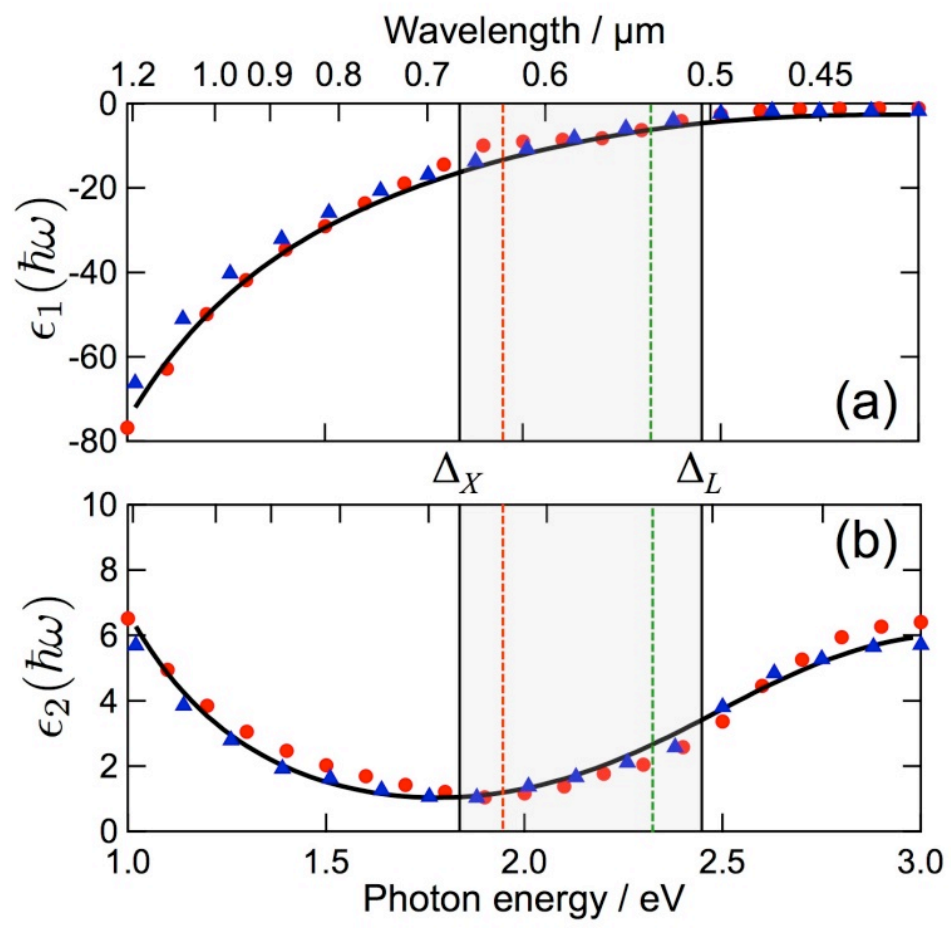

Figure 5.2. Dielectric function data from thin gold films. (a) Real, (b) Imaginary. Red solid circles from [33], blue solid triangles from [38], and solid black line from an analytical model [39]. The shaded gray regions indicate the energy range between $\Delta_{\mathrm{X}}$ and $\Delta_{\mathrm{L}}$. The dashed lines indicate the excitation energies $\hbar \omega_{01}=1.96 \mathrm{eV}$ (red) and $\hbar \omega_{02}=2.33 \mathrm{eV}$ (green).

There have been many reports of plasmon-enhanced single photon photoluminescence from gold nanostructures. Boyd and co-workers described the single-photon plasmonenhanced photoluminescence intensity, due to the enhancement of the radiative decay rate of the localized $d$-hole and $s p$-electron recombination dipole, as $I_{\mathrm{PL}} \sim\left[L\left(\omega_{\mathrm{o}}\right)\right]^{2}\left[L\left(\omega_{\mathrm{PL}}\right)\right]^{2}$, where $L$ is a local surface plasmon field factor that accounts for the enhancement of the excitation and emission fields after crossing the metal interface [5]. In this case, the excitation quantum efficiency $q_{\mathrm{E}}$, and the recombination quantum efficiency $q_{\mathrm{D}}$, are both enhanced with respect to their spectral overlap with the surface plasmon band. An early report of plasmon-enhanced photoluminescence from spherical gold colloids, with diameters less than about $5 \mathrm{~nm}$, excited 
with ultraviolet radiation $(5.39 \mathrm{eV})$ indicated that the $Q_{\mathrm{E}}$ was enhanced by several orders of magnitude with a peak emission energy of $2.82 \mathrm{eV}$ [39]. The photoluminescence was attributed to the radiative recombination of $5 d-6 s p$ electron-hole pairs. Mohamed and coworkers reported photoluminescence measurements from gold nanorod assemblies with varying aspect ratios using an excitation energy of $2.6 \mathrm{eV}$ [40]. The scattering spectra consisted of a small peak at approximately $2.4 \mathrm{eV}$, for all nanorod dimensions, which was assigned to the transverse surface plasmon resonance mode. A large peak in the scattering spectra varied from $1.6 \mathrm{eV}$, for the largest aspect ratio, up to $2.1 \mathrm{eV}$, for the smallest aspect ratio, which directly corresponded to plasmon-enhanced photoluminescence peaks at $2.1 \mathrm{eV}$ and $2.3 \mathrm{eV}$, respectively, and it was concluded that the longitudinal surface plasmon resonance is more efficient at enhancing the photoluminescence intensity, thus both the incoming excitation and the outgoing emitted radiation are enhanced, as previously reported [5]. Dulkeith and co-workers later reported plasmon-enhanced photoluminescence from spherical gold nanoparticles using a $3.3 \mathrm{eV}$ excitation source [41]. The reported $Q_{\mathrm{E}}$ was enhanced by several orders of magnitude, and the peak of the photoluminescence band corresponded directly to the peak of the surface plasmon band, which varied from $2.4 \mathrm{eV}$, for $6 \mathrm{~nm}$ diameter particles, up to $2.3 \mathrm{eV}$ for $60 \mathrm{~nm}$ diameter nanoparticles. Since the spectral overlap of the excitation source with the surface plasmon band was very weak, the authors argued that the enhancement of $q_{\mathrm{E}}$ and $q_{\mathrm{D}}$ could not account for the observed large enhancement of $Q_{\mathrm{E}}$. Additionally, the peak positions of photoluminescence and scattering spectra have consistent shifts, thus precluded the possibility that the photoluminescence spectrum simply reflects the joint density of states of $d$-band holes and $s p$-band electrons. The authors proposed a three-step model based on a previously reported model [42], which consists of a process where photoexcited $d$-band holes relax within the $d$-band, then scatter to the conduction band via emission of surface plasmons, which subsequently decay radiatively. More recently, single-photon photoluminescence from gold nanorods was studied using 2.41 $\mathrm{eV}$ and $1.96 \mathrm{eV}$ excitation sources, and nearly perfect spectral overlap of the luminescence and scattering spectra was reported. They reported that the interband transitions and transverse surface plasmons are excited with the $2.41 \mathrm{eV}$ laser source, in contrast to the longitudinal surface plasmon resonance excited by the $1.96 \mathrm{eV}$ laser source, thus was attributed to the fast interconversion between surface plasmons and hot electron pairs, and concluded that the luminescence occurs via emission by the radiative decay of surface plasmons [32]. Yorulmaz, et al. studied single-photon photoluminescence from gold nanorods with different aspect ratios using a $2.6 \mathrm{eV}$ excitation source, and found a strong correlation 
between the scattering and photoluminescence spectra of the same particle, thus confirming the plasmonic contribution to the measured photoluminescence [43]. They observed that emission was dominated by a polarized contribution that coincides with the longitudinal surface plasmon resonance. In addition to the plasmon-modulated photoluminescence, they observed an additional photoluminescence peak near $2.5 \mathrm{eV}$ that was not affected by the surface plasmon resonance. $\mathrm{Hu}$, et al. recently reported a series of experiments from nanopatterned gold disks, and disk-dimers with varying nanogap spacing, using a $2.33 \mathrm{eV}$ excitation source [44]. They observed that the peak photoluminescence emission energy was strongly dependent on the location of the surface plasmon resonance energy, and the peak photoluminescence emission energy was blue shifted (up to $70 \mathrm{meV}$ ) from the peak surface plasmon resonance energy.

Currently, two different models have been proposed to explain the different plasmonenhanced photoluminescence observations [43]. The first model (model-1) involves a local optical field enhancement of the quantum efficiencies, $q_{\mathrm{E}}$ and $q_{\mathrm{D}}$, of a single-photon photoluminescence process when $\hbar \omega_{\mathrm{SPR}} \sim \hbar \omega_{0} \sim \Delta_{\mathrm{X}, \mathrm{L}}[5,7,40]$. In this case, the external field polarizes the nanostructure, thus inducing the collective electron oscillations and the emission of surface plasmons. The origin of model-1, where the excitation and emission channels are separately enhanced, was borne from studies of SERS and SHG [2-4]. In the second model (model-2), the highly localized $d$-hole photoexcitation forms a dipole at $\Delta_{\mathrm{X}}$, or $\Delta_{\mathrm{L}}$, thus polarizing the nanostructure due to dynamically screened electron-electron interactions induced by the nanostructure confinement, thus establishing the collective free electron oscillation and the emission of surface plasmons that can subsequently decay radiatively giving plasmon-enhanced photoluminescence $[32,42,44]$.

\subsection{Plasmon-modulated photoluminescence of gold}

In this chapter, conventional photoluminescence, from planar (unpatterned) as-deposited thin films of polycrystalline gold, is investigated and compared to plasmon-enhanced photoluminescence, from large area gold nanogap resonators. It should be noted that most of the previous reports of plasmon-enhanced photoluminescence of gold were based on nanoparticles, however, the high uniformity of the gold nanowire array surfaces used here have tunable and highly reproducible plasmon resonances, thus enabling such systematic studies. Using laser excitation energies located between $\Delta_{\mathrm{X}}$ and $\Delta_{\mathrm{L}}$, two very different plasmon-enhanced photoluminescence processes are demonstrated that are strongly dependent on the spectral overlap of the peak surface plasmon band (with resonance energy $\hbar \omega_{\text {SPR }}$ ), the excitation energy $\hbar \omega_{0}$, and the interband transitions $\Delta_{\mathrm{X}} \approx 1.9 \mathrm{eV}$ and $\Delta_{\mathrm{L}} \approx 2.5 \mathrm{eV}$. In the last 
section of the article, the relationship between plasmon-enhanced photoluminescence and the background continuum that accompanies the SERS spectra from benzenethiol and 4mercaptopyridine self-assembled monolayers (SAMs) chemisorbed on the gold nanowire array surfaces is examined.

Photoluminescence experiments are done using two different continuous wave (cw) laser sources: helium-neon (He-Ne) with emission energy $1.96 \mathrm{eV}$, and neodymium-doped yttrium aluminum garnet (Nd-YAG) with emission energy $2.33 \mathrm{eV}$, which are both commonly used for plasmonics applications. All measurements are done in an ambient air environment at room temperature. Planar (unpatterned) as-deposited gold layers are fabricated by sputtering and electron beam evaporation, with thicknesses in the range of 40-100 nm, on silicon nitride $(\mathrm{SiN})$ coated silicon $(\mathrm{Si})$ samples.

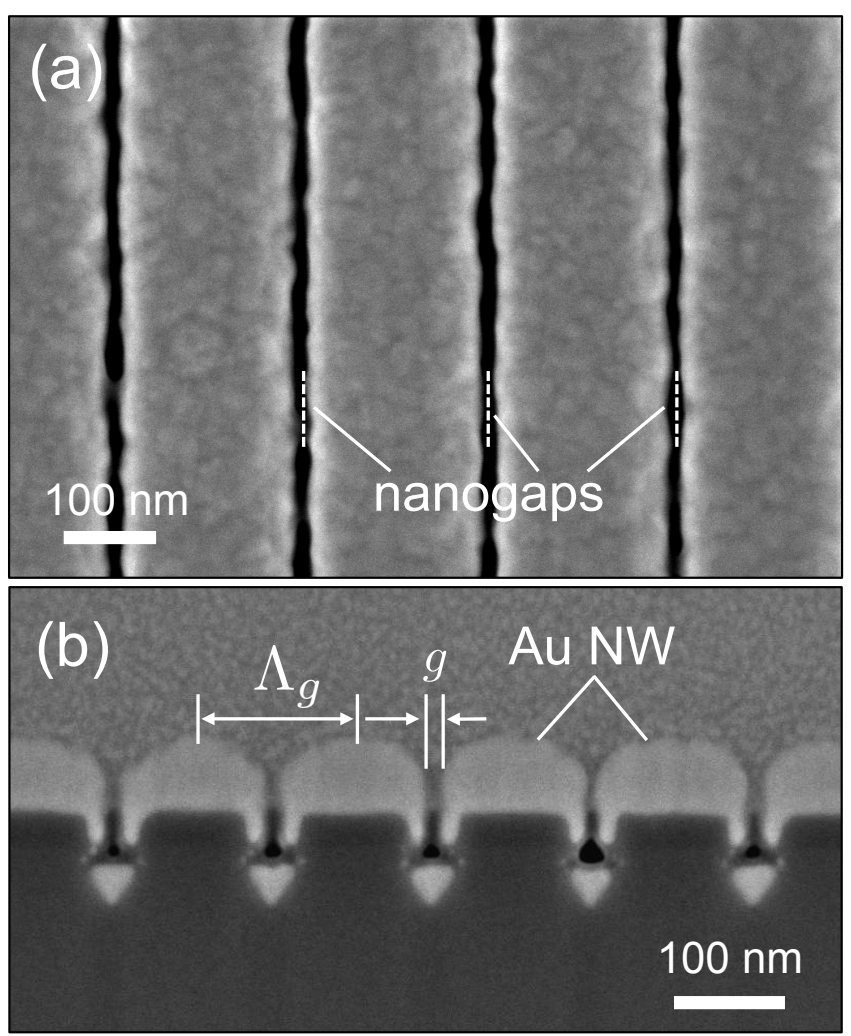

Figure 5.3. Representative SEM images of gold nanowire array surfaces with array pitch $\Lambda_{\mathrm{g}}=200 \mathrm{~nm}$ and nanogap spacing $g=10 \mathrm{~nm}$. (a) Top view, (b) Cross section view.

Gold and silver nanowire array surfaces, previously reported [9,11], are fabricated by sputter coating directly on nanopatterned SiN template surfaces, which results in large area arrays of metal nanowires that are each separated by sub-20 nm nanogaps. Figure 5.3 shows scanning electron microscopy (SEM) images of representative gold nanowire array surfaces used for photoluminescence and SERS measurements. Figures 5.4(a)-(d) show two-dimensional (2D) finite difference time domain (FDTD) numerical simulation results of the electromagnetic fields near the modeled gold nanowire array surface with pitch $\Lambda_{\mathrm{g}}=200 \mathrm{~nm}$ and nanogap 
spacing $g=10 \mathrm{~nm}$. Figures 5.4(a) and 5.4(c) show the gap-mode localized surface plasmon resonance generated in the nanogap regions for p-polarized excitation using $1.96 \mathrm{eV}$ and 2.33 $\mathrm{eV}$ excitation energies, respectively. Figures 5.4(b) and 5.4(d) show that an s-polarized excitation source does not produce the gap-mode localized surface plasmon resonance and essentially behaves as a planar gold surface.
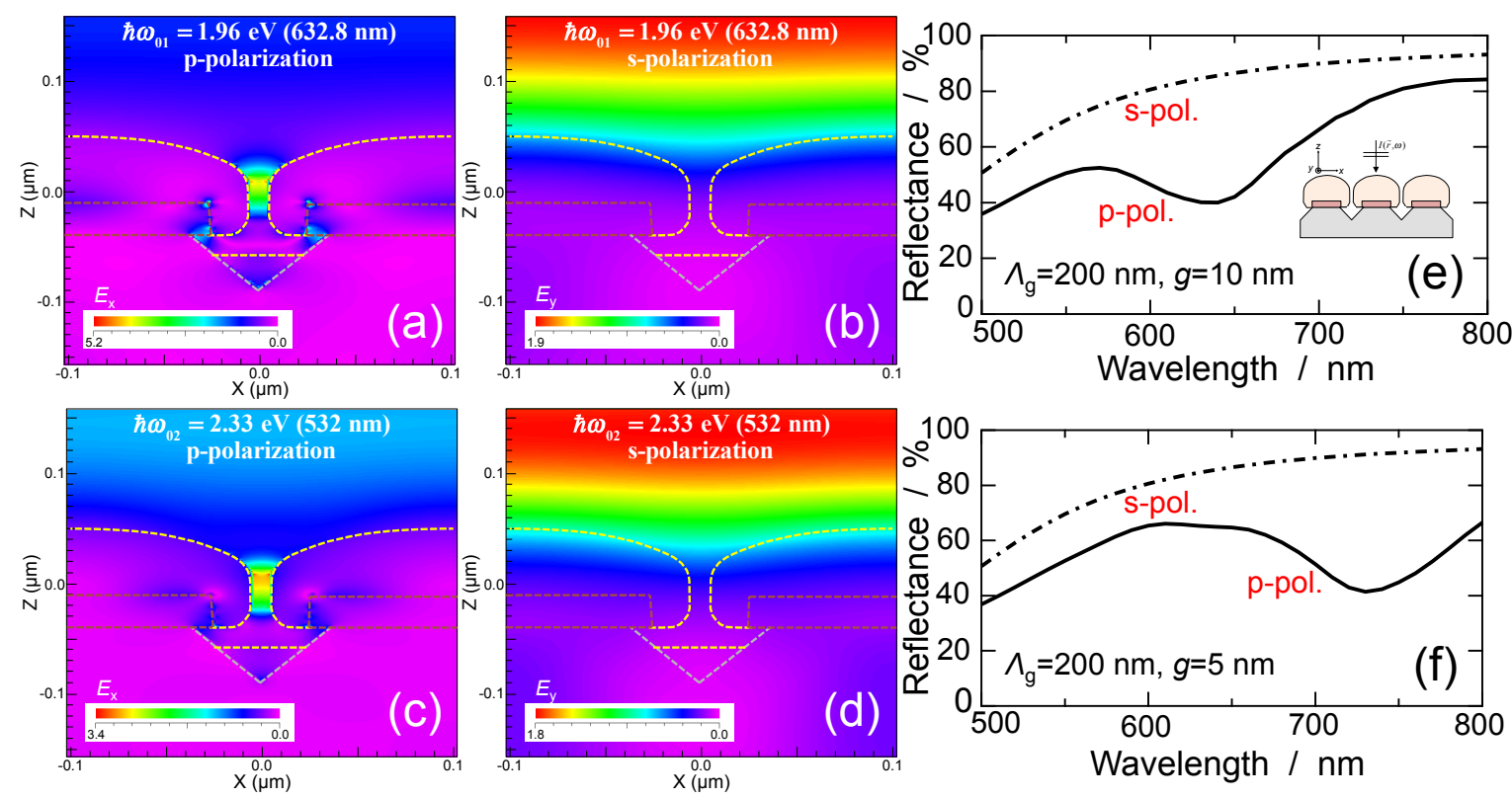

Figure 5.4. (a-d) 2D FDTD simulations of electric field profiles near gold nanogaps for different excitation energy and polarization with $\Lambda_{\mathrm{g}}=200 \mathrm{~nm}$ and $g=10 \mathrm{~nm}$. (yellow dashed lines indicate gold layer; red dashed lines indicate $\mathrm{SiN}$ layer and gray dashed lines indicate the Si layer). (a) Excitation energy $\hbar \omega_{01}=1.96 \mathrm{eV}$ and p-polarization, (b) $\hbar \omega_{01}=1.96 \mathrm{eV}$ and s-polarization, (c) $\hbar \omega_{02}=2.33 \mathrm{eV}$ and ppolarization, (d) $\hbar \omega_{02}=2.33 \mathrm{eV}$ and s-polarization, (e-f) 2D RCWA simulations of reflectance spectra from gold nanogap surfaces with pitch $\Lambda_{\mathrm{g}}=200 \mathrm{~nm}$. Solid lines indicate p-polarized reflectance and dashed-dotted lines indicate s-polarized reflectance, (e) $g=10 \mathrm{~nm}$, (f) $g=5 \mathrm{~nm}$.

The surface plasmon resonance band (with peak energy $\hbar \omega_{\text {SPR }}$ ) of the gold nanowire array surfaces can be precisely tuned throughout the visible spectrum by changing the nanogap pitch $\Lambda_{\mathrm{g}}$ and spacing $g$ [35], which is used to investigate the influence of the plasmon resonance on the photoluminescence emission. Figures 5.4(e) and 5.4(f) show reflectance spectra calculated using 2D rigorous coupled waveguide analysis (RCWA) code. As the nanogap spacing is reduced from $g=10 \mathrm{~nm}$ (Fig. 5.4(e)) to $g=5 \mathrm{~nm}$ (Fig. 5.4(f)), the surface plasmon resonance wavelength (the minimum reflectance located at the dip of the reflectance spectrum) from p-polarized excitation red shifts, which is consistent with previous reports $[35,36]$. Incident s-polarized excitation does not induce a localized surface plasmon resonance, which is consistent with FDTD simulations and experimental reflectance measurements. In subsequent experiments, the plasmon-enhanced photoluminescence is investigated by varying the nanogap spacing, and thus the localized surface plasmon 
resonance energy, which is used to investigate the characteristics of plasmon-enhanced photoluminescence from the gold nanowire array surfaces. Three metrics are defined to aid in quantifying the spectral overlaps of $\hbar \omega_{0}, \hbar \omega_{\mathrm{SPR}}, \Delta_{\mathrm{X}}$, and $\Delta_{\mathrm{L}}$. The energy difference of the peak surface plasmon resonance energy and the excitation energy is indicated as $\delta_{\mathrm{E}}=\hbar\left(\omega_{\mathrm{SPR}}-\omega_{0}\right)$. The energy differences of the peak surface plasmon resonance energy and the interband transition energies are indicated as $\delta_{\mathrm{SX}}=\hbar \omega_{\mathrm{SPR}}-\Delta_{\mathrm{X}}$ and $\delta_{\mathrm{SL}}=\hbar \omega_{\mathrm{SPR}}-\Delta_{\mathrm{L}}$. Small values of $\delta_{\mathrm{E}}, \delta_{\mathrm{SX}}$, and $\delta_{\mathrm{SL}}$ indicate strong spectral overlaps, and vice versa.

\subsubsection{He-Ne laser excitation}

Photoluminescence spectra generated with a focused cw He-Ne laser source $(1.96 \mathrm{eV})$ of varying laser power levels from planar $100 \mathrm{~nm}$ thick gold layers are shown in Fig. 5.5. The maximum power density of the focused laser source is $0.7 \mathrm{MW} \mathrm{cm}^{-2}(4 \mathrm{~mW}$ power).
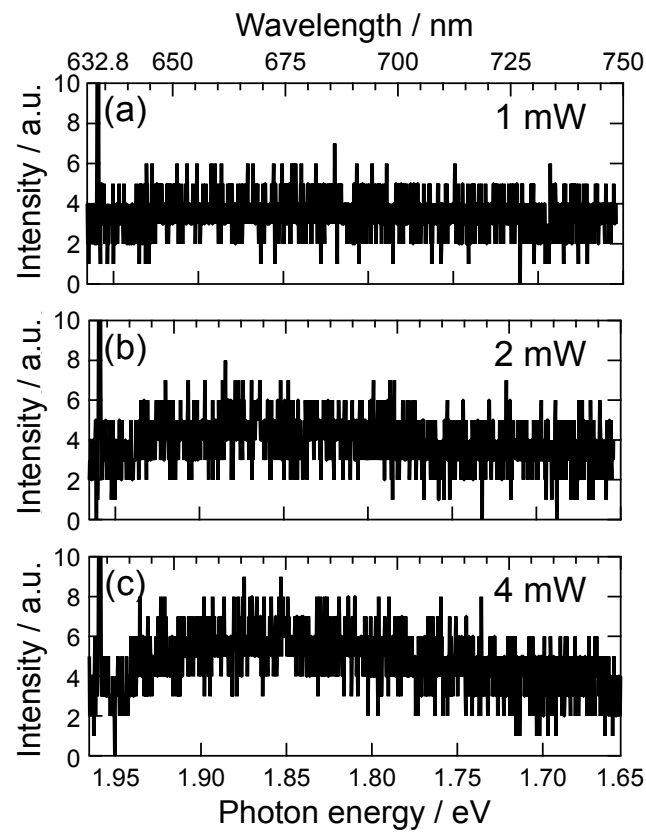

Figure 5.5. Representative photoluminescence spectra from planar as-deposited gold layers excited with a He-Ne laser $\left(\hbar \omega_{01}=1.96 \mathrm{eV}\right)$ at different power levels. (a) $1 \mathrm{~mW}$. (b) $2 \mathrm{~mW}$, (c) $4 \mathrm{~mW}$. A 100 ms integration time was used for all measurements.

The photoluminescence from planar gold layers is not dependent on the polarization state of the laser source and the average peak photoluminescence intensity increases linearly with laser power, thus ensuring a single-photon absorption process. Low laser powers and short integration times are used because the plasmon-enhanced photoluminescence responses commonly observed in SERS spectra are measured using similar conditions, and additionally, to avoid sample heating The peak photoluminescence intensity from planar gold layers is very weak, which is consistent with previous reports of small $Q_{\mathrm{E}}$ for thin gold films $[1,5,8]$. The peak photoluminescence energy from the planar gold layers is $\hbar \omega_{\mathrm{PL}} \approx(1.86 \pm 0.02) \mathrm{eV}$, which correlates well with previously reported values and is consistent with radiative recombination 
of $5 d-6 s p$ interband transitions near $\Delta_{\mathrm{X}}[5,28,29]$. The photoluminescence measurements show a tail-like distribution on the low-energy side of the spectrum.

Figure 5.6 shows representative reflectance and photoluminescence spectra from gold nanowire array surfaces. Different peak surface plasmon resonance bands are shown in the reflectance spectra (surface $S_{1}$, Fig. 5.6(a); surface $S_{2}$, Fig. 5.6(c); and surface $S_{3}$, Fig. 5.6(e)). The surface plasmon resonance band is varied by changing the nanogap spacing $g$, as shown in simulation results of Figs. 5.4(e) and 5.4(f).
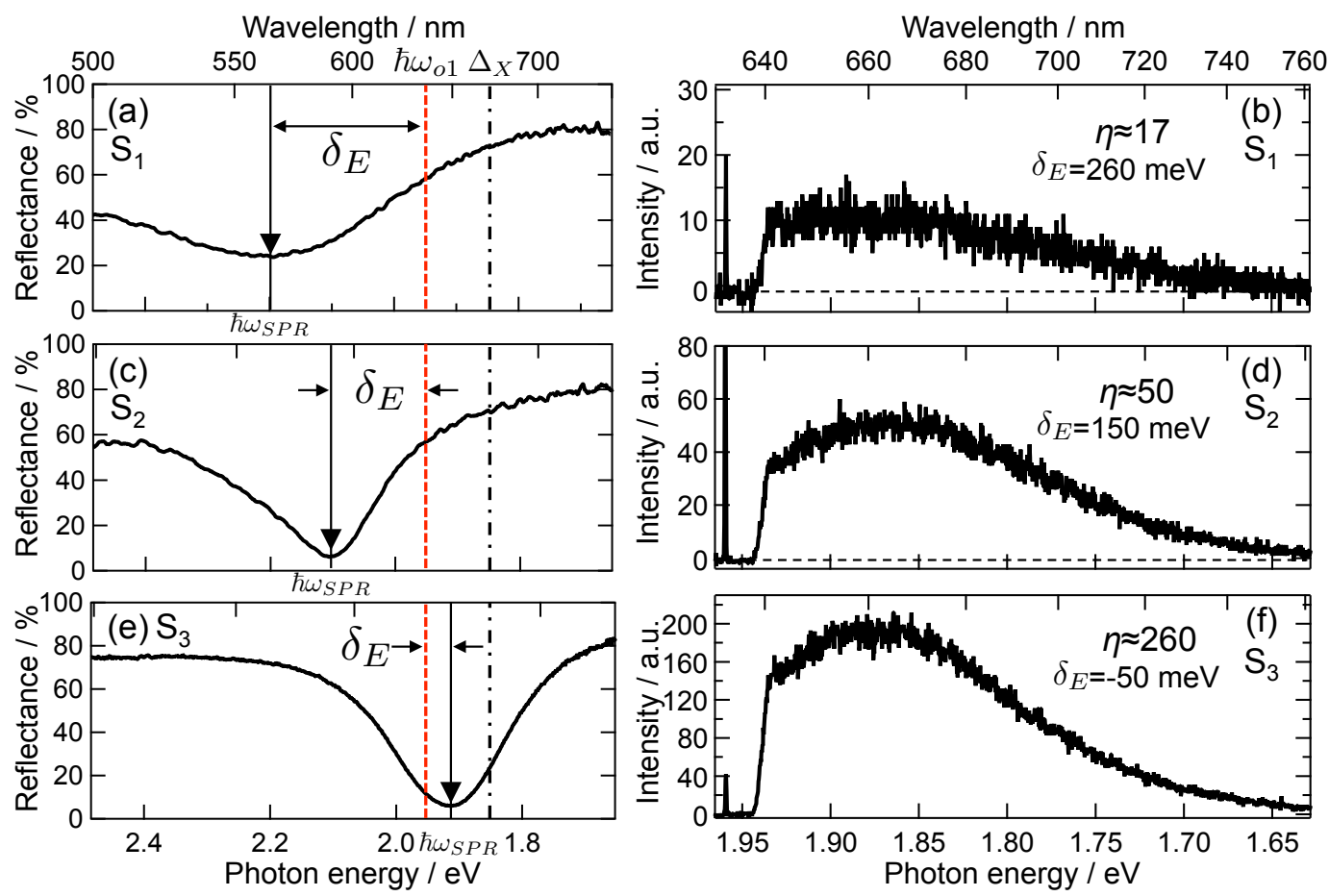

Figure 5.6. Representative reflectance and photoluminescence spectra from three different gold nanowire array surfaces each with different surface plasmon resonance energy $\hbar \omega_{\mathrm{SPR}}$. The excitation energy $(1.96 \mathrm{eV})$ is indicated with a dashed red line. (a),(b) Surface $\mathrm{S}_{1}$ with $\delta_{\mathrm{E}} \approx 260 \mathrm{meV}$, (c),(d) Surface $\mathrm{S}_{2}$ with $\delta_{\mathrm{E}} \approx 150 \mathrm{meV}$, (e),(f) Surface $\mathrm{S}_{3} \delta_{\mathrm{E}} \approx-50 \mathrm{meV}$. A He-Ne laser excitation source with 1 $\mathrm{mW}$ power and $100 \mathrm{~ms}$ integration time was used for all measurements. Note the different intensity scales.

The experimental reflectance spectra are generated using p-polarized white light and the photoluminescence spectra are generated using a p-polarized He-Ne laser. Since the He- $\mathrm{Ne}$ laser excitation energy $\left(\hbar \omega_{01}=1.96 \mathrm{eV}\right)$ is located on the high energy side of $\Delta_{\mathrm{X}}$, the plasmonenhanced photoluminescence is due to interband transitions near $\Delta_{\mathrm{X}}[5,27,29]$. The peak photoluminescence energy $\hbar \omega_{\mathrm{PL}}$ from all three of the nanostructured surfaces remains approximately constant at $\left(\hbar \omega_{\mathrm{PL}}=1.87 \pm 0.01\right) \mathrm{eV}$ (the peak emission energies were calculated from $n=50$ measurements from each surface. For surface $\mathrm{S}_{1}\left(\hbar \omega_{\mathrm{SPR}}=2.2 \mathrm{eV}\right)$ : $\hbar \omega_{\mathrm{PL}}=1.872 \pm 0.006 \mathrm{eV}(663.3 \pm 2.2 \mathrm{~nm}) ;$ surface $\mathrm{S}_{2}\left(\hbar \omega_{\mathrm{SPR}}=2.1 \mathrm{eV}\right): \hbar \omega_{\mathrm{PL}}=1.863 \pm 0.008 \mathrm{eV}$ $(665.3 \pm 2.8 \mathrm{~nm}) ;$ and surface $\left.\mathrm{S}_{3}\left(\hbar \omega_{\mathrm{SPR}}=1.9 \mathrm{eV}\right): \hbar \omega_{\mathrm{PL}}=1.871 \pm 0.003 \mathrm{eV}(662.6 \pm 2.3 \mathrm{~nm})\right)$, 
which is blue-shifted from the peak photoluminescence from the planar gold surfaces by about $10 \mathrm{meV}$, but more importantly $\hbar \omega_{\mathrm{PL}}$ does not shift as the spectral overlap of $\hbar \omega_{\mathrm{SPR}}$ with $\hbar \omega_{01}$ is varied, i.e. as $\delta_{\mathrm{E}}$ is varied from large $(260 \mathrm{meV})$ to small $(-50 \mathrm{meV})$ values (Figs. 5.6(b), 5.6(d), and 5.6(f)). The emission band shape does change as a function of the spectral overlap. The peak photoluminescence intensity is strongly modulated as the spectral overlap is varied. For $\delta_{\mathrm{E}} \approx 260 \mathrm{meV}$ (the weakest spectral overlap), the peak photoluminescence intensity is enhanced by a factor of $\eta \approx 17$, where $\eta$ is defined as the ratio of the peak plasmonmodulated photoluminescence intensity to the photoluminescence intensity measured from a planar gold layer.
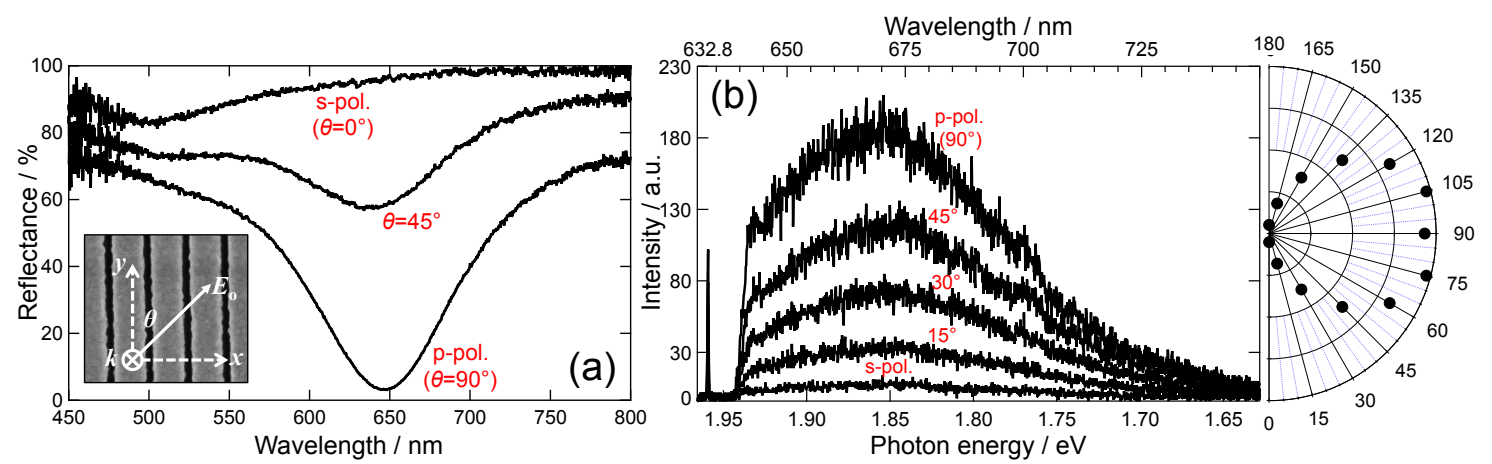

Figure 5.7. (a) Reflectance spectra from a representative gold nanowire array surface $\left(\Lambda_{\mathrm{g}}=200 \mathrm{~nm}\right.$, $g=10 \mathrm{~nm}$ ) for different incident light polarizations (p-polarization: electric field polarization perpendicular to the length of the nanogap, and s-polarization: electric field polarization parallel to the length of the nanogap), (b) Photoluminescence spectra as the incident polarization alignment relative to the length of the nanogap structure is varied (inset: laser polarization alignment to nanogap structure). Right: Polar plot of the peak photoluminescence for different polarization alignments.

For $\delta_{\mathrm{E}} \approx 150 \mathrm{meV}$ (increased spectral overlap), the photoluminescence is further enhanced to $\eta \approx 50$ (Figs. 5.6(c) and 5.6(d)), and as the spectral overlap is increased, with $\delta_{\mathrm{E}} \approx-50 \mathrm{meV}$, the photoluminescence enhancement factor is further increased to $\eta \approx 260$. The peak plasmonmodulated photoluminescence enhancement $(\eta \approx 350)$ occurs near the excitation laser energy $\hbar \omega_{01}$. This photoluminescence enhancement is of the same order of magnitude as the simulated localized electromagnetic field enhancement generated in the nanogap regions with a p-polarized plane wave $\left(\hbar \omega_{01}=1.96 \mathrm{eV}\right)$ shown in Fig. 5.4(a). Since the photoluminescence emission energy does not shift as $\delta_{\mathrm{E}}$ is varied, and the photoluminescence intensity is strongly enhanced for a strong spectral overlap ( $\operatorname{small} \delta_{\mathrm{E}}$ ), the photoluminescence process, in this case, follows model-1, which is due to radiative recombination of $5 d$-holes with $6 s p$-electrons near $\Delta_{\mathrm{X}}$, and enhanced photoluminescence due to the enhancement of $q_{\mathrm{E}}$ and $q_{\mathrm{D}}$ by a local field enhancement via a localized surface plasmon resonance in the nanogap region [5]. Since the $d$-band holes are strongly localized near the $X$-symmetry point, this process can be considered to be resonantly enhanced dipole radiation. 
Figure 5.7 shows the polarization dependence of the reflectance and photoluminescence spectra generated from the gold nanowire array surfaces. For s-polarized incident light $\left(\theta=0^{\circ}\right)$, the reflectance from the nanostructured surface, shown in Fig. 5.7(a), does not exhibit a localized surface plasmon resonance in the nanogap regions (i.e. no dip in the reflectance spectrum) and behaves approximately as a planar gold surface, which is consistent with the FDTD simulation results (Figs. 5.4(b) and 5.4(d)), and the RCWA simulation results (Figs. 5.4(e) and 5.4(f)). For p-polarized incident light $\left(\theta=90^{\circ}\right)$, the reflectance from the nanostructured surface exhibits a strong localized surface plasmon resonance in the nanogap regions with a large reflectance dip $\left(\hbar \omega_{\mathrm{SPR}} \approx 1.92 \mathrm{eV}\right)$ in the reflectance spectra, which is consistent with the FDTD simulation results (Figs. 5.4(a)). Figure 5.7(b) shows photoluminescence spectra from a gold nanowire array surface as the laser excitation (He-Ne, $\hbar \omega_{01} \approx 1.96 \mathrm{eV}$ ) polarization is varied from s-polarization state to p-polarization state. For spolarized incident excitation, the photoluminescence is very weak, which indicates no plasmon enhancement of the photoluminescence, and is consistent with the experimental reflectance spectra (Fig. 5.7(a)) and simulation results (Fig. 5.4(b)). The photoluminescence intensity from the p-polarized excitation is strongly enhanced, which is consistent with the generation of a localized surface plasmon resonance in the nanogap region, as shown in the experimental reflectance spectra (Fig. 7(a)) and the simulation results (Figs. 5.4(a) and 5.4(e)). In this case, $\hbar \omega_{\mathrm{SPR}} \approx 1.92 \mathrm{eV}$ is located between $\hbar \omega_{\mathrm{PL}} \approx 1.86 \mathrm{eV}$ and $\hbar \omega_{01} \approx 1.96 \mathrm{eV}$, thus indicating that both channels are strongly enhanced according to model-1, where the photoluminescence intensity is enhanced by a local field enhancement of the excitation (with quantum efficiency $q_{\mathrm{E}}$ ) and emission (with efficiency $q_{\mathrm{D}}$ ) fields crossing the metal interface as $I_{\mathrm{PL}} \sim\left[L\left(\omega_{01}\right)\right]^{2}\left[L\left(\omega_{\mathrm{PL}}\right)\right]^{2}[5]$. These measurements, in combination with the simulation results, support the enhancement of $Q_{\mathrm{E}}$ as the mechanism of the plasmon-enhanced photoluminescence for $\hbar \omega_{01} \sim \hbar \omega_{\mathrm{SPR}} \sim \Delta_{\mathrm{L}}$.

\subsubsection{Nd-YAG laser excitation}

Photoluminescence spectra generated with a focused Nd-YAG laser source $(2.33 \mathrm{eV})$ of varying laser power from planar $100 \mathrm{~nm}$ thick gold layers are shown in Fig. 5.8. The maximum power density of the focused laser source is $0.9 \mathrm{MW} \mathrm{cm}{ }^{-2}$ ( $4 \mathrm{~mW}$ power). The average peak photoluminescence intensity increases linearly with laser power. The photoluminescence from planar gold layers is not dependent on the polarization state of the laser source and the average peak photoluminescence intensity increases linearly with laser power. The average peak photoluminescence emission energy from planar gold thin films is 
$\hbar \omega_{\mathrm{PL}} \approx 2.24 \mathrm{eV}$, and the peak photoluminescence intensity is approximately $20 \times$ greater than the photoluminescence generated by the He-Ne laser source. Since the $2.33 \mathrm{eV}$ excitation energy is less than $\Delta_{\mathrm{L}}$ (Fig. 5.1(b)), interband transitions near $\Delta_{\mathrm{L}}$ are not expected to contribute to the photoluminescence. However, previously reported photoluminescence spectra produced with a similar excitation energy from planar gold surfaces showed a photoluminescence emission energy near $2.2 \mathrm{eV}$, which was attributed to interband transitions near $\Delta_{\mathrm{L}}$ [5]. The same authors reported a peak photoluminescence energy near $2.2 \mathrm{eV}$, produced with a much larger excitation energy $(4.67 \mathrm{eV})$, and assigned the emission to a combination of $5 d-6 s p$ transitions near $\Delta_{\mathrm{L}}$ and $4 d-6 s p$ transitions near $\Delta_{\mathrm{X}}$ [5]. However it is unlikely that $4 d-6 s p$ transitions near $\Delta_{\mathrm{X}}$ contribute to the photoluminescence in this case.
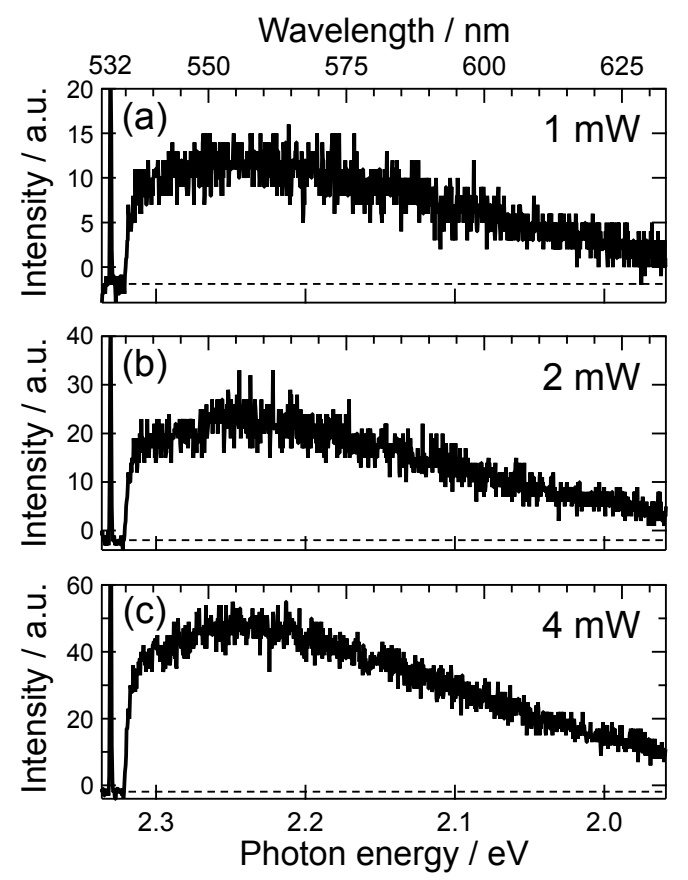

Figure 5.8. Photoluminescence spectra from planar gold layers using a Nd-YAG laser source (2.33 $\mathrm{eV}$ ) at different power levels. (a) $1 \mathrm{~mW}$, (b) $2 \mathrm{~mW}$, (c) $4 \mathrm{~mW}$. Note the different intensity scales. A $100 \mathrm{~ms}$ integration time was used for all measurements.

The contribution of the $\Delta_{\mathrm{X}}$ and $\Delta_{\mathrm{L}}$ interband transitions to the photoluminescence generated with the Nd-YAG laser becomes more evident from the measurements of nanostructured gold surfaces, where the photoluminescence emission characteristics are significantly modified depending on the spectral overlap of $\hbar \omega_{0}, \hbar \omega_{\text {SPR }}$, and $\Delta_{X}$.

Figure 5.9 shows representative reflectance and photoluminescence spectra from gold nanowire array surfaces with different $\hbar \omega_{\text {SPR. }}$. For all three surfaces, the spectral overlap of the excitation source and surface plasmon band is very weak with $\delta_{\mathrm{E}} \approx-410 \mathrm{meV}$ for surface $\mathrm{S}_{1}$ (Figs. 5.9(a)), $\delta_{\mathrm{E}} \approx-280 \mathrm{meV}$ for $\mathrm{S}_{2}$ (Figs. 5.9(c)), and $\delta_{\mathrm{E}} \approx-650 \mathrm{meV}$ for $\mathrm{S}_{3}$ (Figs. 5.9(e)), and therefore, it is not expected that the external field can induce a strong surface plasmon 
resonance, thus $q_{\mathrm{E}}$ is not significantly enhanced [41]. Despite the weak spectral overlap of $\hbar \omega_{02}$, with $\hbar \omega_{\mathrm{SPR}}$, the peak photoluminescence intensity and emission energy are significantly modified. For surface $S_{1}$, the peak surface plasmon resonance energy is $\hbar \omega_{\mathrm{SPR}} \approx 1.92 \mathrm{eV}$, and therefore, $\delta_{\mathrm{SX}} \approx 60 \mathrm{meV}$, thus there is a strong spectral overlap of $\hbar \omega_{\mathrm{SPR}}$ with $\Delta_{\mathrm{X}}$. The ppolarized excitation results in a peak photoluminescence emission of $\hbar \omega_{\mathrm{PL}, \mathrm{p}} \approx 1.98 \mathrm{eV}$ that is blue shifted from $\hbar \omega_{\text {SPR }}$ by about $60 \mathrm{meV}$.
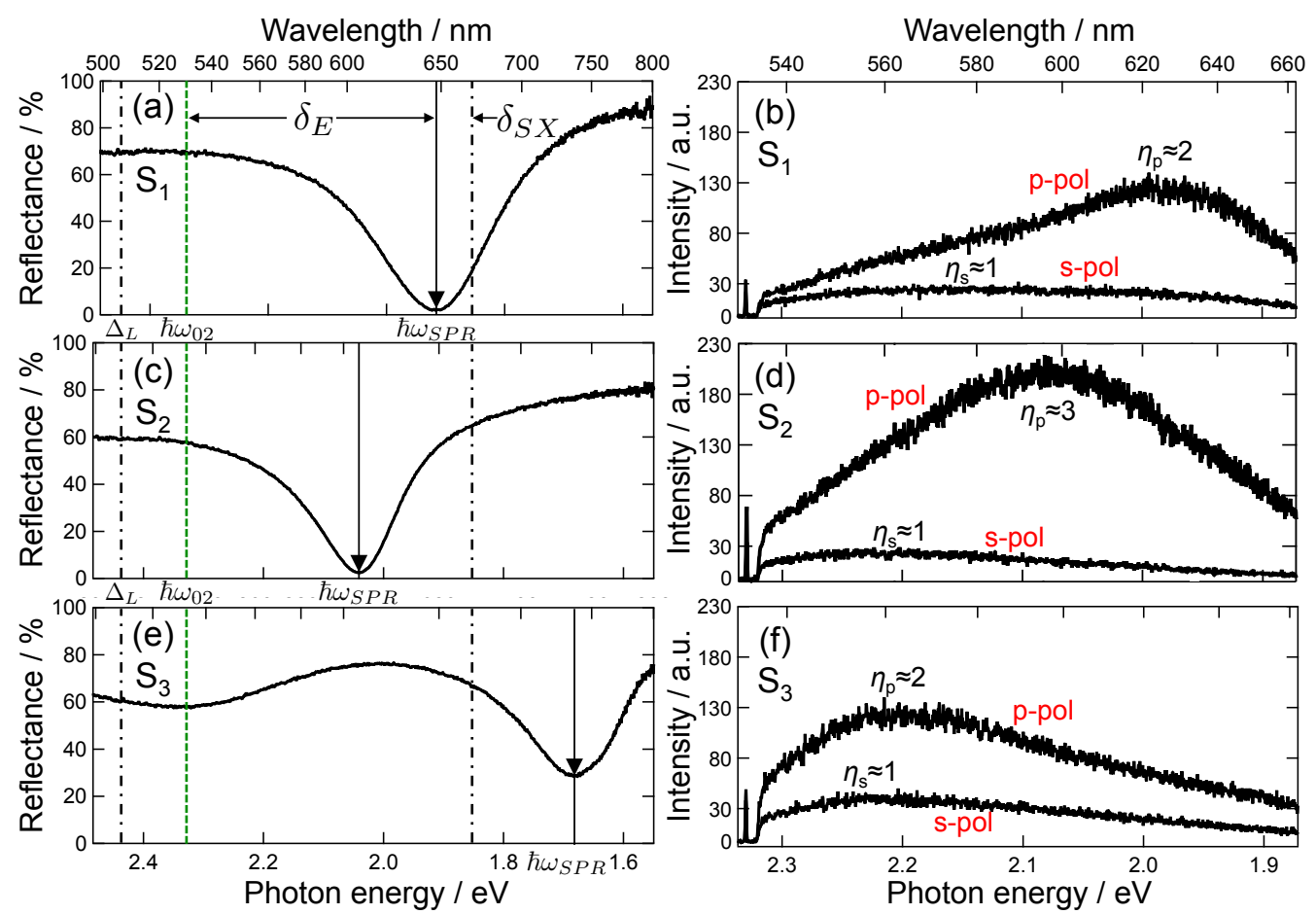

Figure 5.9. Reflectance and photoluminescence spectra from three different gold nanowire array surfaces $\left(\Lambda_{\mathrm{g}}=200 \mathrm{~nm}\right.$ and different $\left.g\right)$ each with different surface plasmon resonance energy $\hbar \omega_{\text {SPR }}$. The excitation energy $(2.33 \mathrm{eV})$ is indicated with a dashed green line. The location of the interband transitions $\Delta_{\mathrm{X}}$ and $\Delta_{\mathrm{L}}$ are indicated with dashed-dotted lines. (a),(b) Surface $\mathrm{S}_{1}$ with $\delta_{\mathrm{E}}=-410 \mathrm{meV}$ and $\delta_{\mathrm{SX}} \approx 60 \mathrm{meV}$, p-polarized emission peak $\hbar \omega_{\mathrm{PL}, \mathrm{p}} \approx 1.98 \mathrm{eV}$, and s-polarized emission peak $\hbar \omega_{\mathrm{PL}, \mathrm{s}} \approx 2.2 \mathrm{eV}$, (c),(d) Surface $\mathrm{S}_{2}$ with $\delta_{\mathrm{E}} \approx-280 \mathrm{meV}$ and $\delta_{\mathrm{SX}} \approx 190 \mathrm{meV}$, p-polarized emission peak $\hbar \omega_{\mathrm{PL}, \mathrm{p}} \approx 2.08 \mathrm{eV}$, and s-polarized emission peak $\hbar \omega_{\mathrm{PL}, \mathrm{s}} \approx 2.2 \mathrm{eV}$, (e),(f) Surface $\mathrm{S}_{3}$ with $\delta_{\mathrm{E}} \approx-650 \mathrm{meV}$ and $\delta_{\mathrm{Sx}} \approx-180 \mathrm{meV}$, p-polarized emission peak $\hbar \omega_{\mathrm{PL}, \mathrm{p}} \approx 2.2 \mathrm{eV}$, and s-polarized emission peak $\hbar \omega_{\mathrm{PL}, \mathrm{s}} \approx 2.2 \mathrm{eV}$. All measurements conducted with the Nd-YAG laser power of $0.1 \mathrm{~mW}$ with a $3 \mathrm{~s}$ integration time.

The peak intensity is enhanced by a factor of $\eta_{\mathrm{p}} \approx 2$, compared to the planar gold surfaces. The peak photoluminescence intensity and emission energy $\left(\hbar \omega_{\mathrm{PL}, \mathrm{z}} \approx 2.2 \mathrm{eV}\right)$ generated with the spolarized excitation are similar to the conventional photoluminescence from a planar gold surface, which is supported by the FDTD simulation results (Fig. 5.4(d)). For surface $\mathrm{S}_{2}$, $\hbar \omega_{\mathrm{SPR}} \approx 2.05 \mathrm{eV}$ and $\delta_{\mathrm{SX}} \approx 190 \mathrm{meV}$, thus the spectral overlap of $\hbar \omega_{\mathrm{SPR}}$ with $\Delta_{\mathrm{X}}$ is reduced. The peak photoluminescence intensity generated with the s-polarized excitation is not enhanced and the peak emission energy is $\hbar \omega_{\mathrm{PL}, \mathrm{s}} \approx 2.2 \mathrm{eV}$, thus similar to the photoluminescence characteristics of a planar gold surface, which is also consistent with the FDTD simulation 
results (Fig. 5.4(d)). The p-polarized excitation results in a peak photoluminescence emission energy of $\hbar \omega_{\mathrm{PL}, \mathrm{p}} \approx 2.08 \mathrm{eV}$, which is blue shifted from $\hbar \omega_{\mathrm{SPR}}$ by about $30 \mathrm{meV}$. The peak intensity is enhanced by a factor of $\eta_{\mathrm{p}} \approx 3$. The photoluminescence spectra from surface $\mathrm{S}_{1}$ and $\mathrm{S}_{2}$ (Figs. 5.9(a)-9(d)), are similar to those reported by $\mathrm{Hu}$, et al. [43], however, the interpretation of the mechanism described here is slightly different. When the peak surface plasmon resonance energy $\hbar \omega_{\mathrm{SPR}}$ is located between $\Delta_{\mathrm{X}}$ and $\Delta_{\mathrm{L}}$, and is varied, the resulting peak photoluminescence energy directly correlates with the movement of $\hbar \omega_{\text {SPR }}$ accompanied by a small blue shift. In these cases, model-2 is plausible, which is based on the localized dipole near $\Delta_{\mathrm{X}}$ polarizing the nanostructure, thus exciting the emission of surface plasmons that subsequently decay radiatively near $\hbar \omega_{\text {SPR }}[41,42]$. Additionally, the shape of the photoluminescence spectrum from surface $S_{2}$ with p-polarized excitation is drastically different compared to the other spectra. The total photoluminescence in this case may be due to emission by surface plasmons due to the localized dipole at $\Delta_{\mathrm{X}}$, as well as emission from radiative recombination near $\Delta_{\mathrm{L}}(\approx 2.2 \mathrm{eV})$, which is possible because the surface plasmon resonance band is centrally located and partially overlaps both interband transitions. Dual photoluminescence emission peaks from gold nanorods have been previously reported [43]. For surface $\mathrm{S}_{3}, \hbar \omega_{\mathrm{SPR}}=1.68 \mathrm{eV}, \delta_{\mathrm{SX}} \approx-180$, thus the spectral overlap of $\hbar \omega_{\mathrm{SPR}}$ with $\Delta_{\mathrm{X}}$ is shifted to the low energy side of $\Delta_{\mathrm{X}}$. With the weak spectral overlap of $\hbar \omega_{\text {SPR }}$ with $\Delta_{\mathrm{X}}$, the peak photoluminescence emission shifts back to $\hbar \omega_{\mathrm{PL}, \mathrm{p}} \approx 2.2 \mathrm{eV}$, which is similar to emission from planar gold surfaces. Since the spectral overlap of the excitation source and the surface plasmon band is very weak (large $\delta_{\mathrm{E}}$ ), $q_{\mathrm{E}}$ will not be enhanced. The photoluminescence spectra generated with the s-polarized excitation is also similar to that from the planar gold surface. In addition, the photoluminescence emission also contains generation from the $5 d$ $6 s p$ transition near $\Delta_{\mathrm{L}}$, as was measured from the planar gold surfaces, thus accounting for the gradual intensity decrease on the high energy side of the peak intensity, which was also present in previous reports $[43,44]$.

\subsection{Plasmon-enhanced photoluminescence and SERS}

Plasmon-enhanced photoluminescence plays a significant role in the SERS background continuum from gold nanostructures, which is shown by directly comparing the photoluminescence spectra from unmodified gold nanowire array surfaces, similar to those presented earlier, to SERS spectra measured from gold nanowire array surfaces modified with benzenethiol and 4-mercaptopyridine SAMs. The photoluminescence and SERS spectra are generated on gold nanowire array surfaces with a focused $\mathrm{He}-\mathrm{Ne}(1.96 \mathrm{eV}) \mathrm{cw}$ laser source. 
Figure 5.10 shows representative photoluminescence spectra measured from gold nanowire array surfaces fabricated with a strong spectral overlap of $\hbar \omega_{01}=1.96 \mathrm{eV}$ with $\hbar \omega_{\mathrm{SPR}}\left(\delta_{\mathrm{E}} \approx-74\right.$ $\mathrm{meV})$. The average peak plasmon-enhanced photoluminescence emission energy is $\hbar \omega_{\mathrm{PL}} \approx 1.84$ $\mathrm{eV}$, and the average peak intensity increases linearly with the laser excitation power.

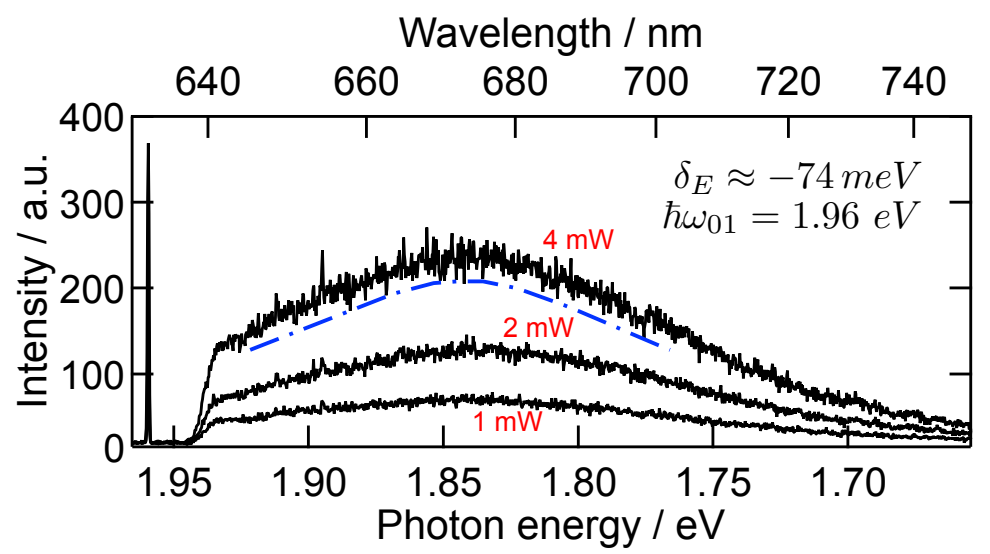

Figure 5.10. Photoluminescence spectra from unmodified gold nanowire array surface excited with a $\mathrm{He}-\mathrm{Ne}$ laser source. A $100 \mathrm{~ms}$ integration time was used for all measurements. Blue dashed-dotted line highlights the general background continuum profile.

The corresponding SERS spectra from the benzenethiol SAM modified gold nanowire array surface is shown in Figure 5.11. The Raman vibration bands of benzenethiol chemisorbed on the gold nanowire array surfaces are consistent with previous assignments ${ }^{3}$ [47]. The vibration band intensities increase linearly with an increasing laser power as expected. The strong correlation between the plasmon-enhanced photo-luminescence band shape and the SERS background continuum is clearly evident.

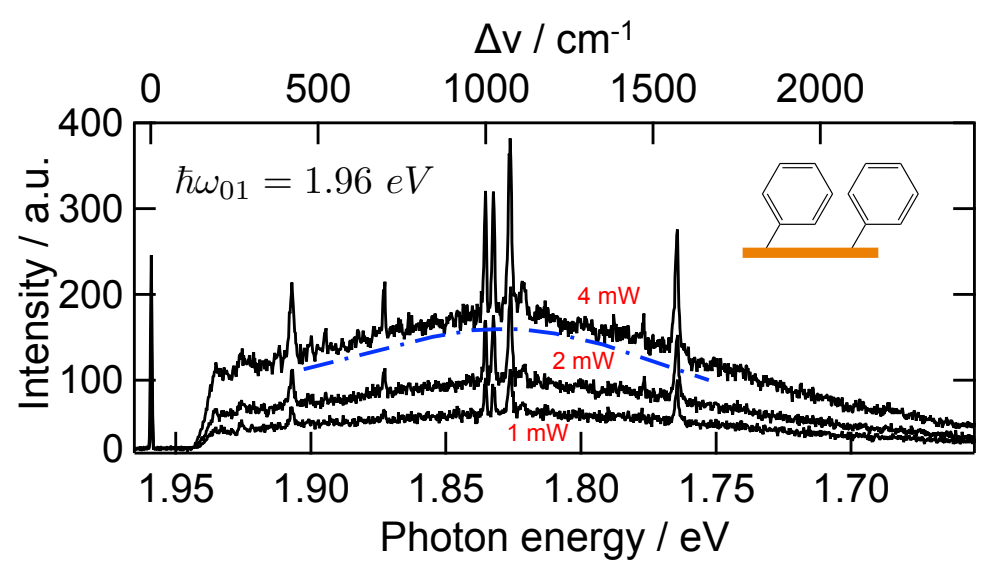

Figure 5.11. SERS spectra from a gold nanowire array surface modified with benzenethiol SAM. Raman shift $\Delta v$ scale located on the top axis. A He-Ne laser source with 100 ms integration time was used for all measurements. Blue dashed-dotted line highlights the general background continuum profile.

\footnotetext{
${ }^{3}$ Raman vibration bands of benzenethiol SAM modified gold nanowire array surfaces are $7 \mathrm{a}\left(\mathrm{a}_{1}\right), \mathrm{v}(\mathrm{C}-\mathrm{S})+\beta(\mathrm{C}-$ C-C) $\left(421 \mathrm{~cm}^{-1}\right) ; 6 a\left(a_{1}\right), \beta(C-C-C)+v(C-S)\left(696 \mathrm{~cm}^{-1}\right) ; 12\left(a_{1}\right), \beta(C-C-C)\left(999 \mathrm{~cm}^{-1}\right) ; 18 a\left(a_{1}\right), \beta(C-H)(1023$ $\left.\mathrm{cm}^{-1}\right) ; 1\left(\mathrm{a}_{1}\right), \beta(\mathrm{C}-\mathrm{C}-\mathrm{C})+\mathrm{v}(\mathrm{C}-\mathrm{S})\left(1072 \mathrm{~cm}^{-1}\right)$; and $8 \mathrm{a}\left(\mathrm{a}_{1}\right), \mathrm{v}(\mathrm{C}-\mathrm{C})\left(1570 \mathrm{~cm}^{-1}\right)$.
} 
Compared to the photoluminescence measurements (Fig. 5.10), the peak background continuum intensity is reduced by approximately 30\%, and the average peak background emission energy is reduced to $\hbar \omega_{\mathrm{PL}} \approx 1.83 \mathrm{eV}$. The reduction in the peak photoluminescence intensity in the SERS spectrum can be explained by the fact that the surface plasmon resonance band is red-shifted due to the increased dielectric constant of benzenethiol on the gold surface $(\varepsilon \sim 2.3)$ compared to an unmodified gold surface. The increased dielectric constant results in decreased $\hbar \omega_{\text {SPR }}$, thus in a reduced spectral overlap with $\hbar \omega_{01}$ (increased $\left.\delta_{\mathrm{E}}\right)$ and reduction in the peak photoluminescence intensity, as shown in Fig. 5.6. The shift of the peak emission $\hbar \omega_{\mathrm{PL}}$ to a lower energy $(\sim 10 \mathrm{meV})$ can be explained by considering a reduction in photoluminescence energy induced by the increased dielectric constant at the gold surface where the emission energy is determined by the metal interface to the surrounding medium.

Figure 5.12 shows a SERS spectrum from a gold nanowire array surface modified with a 4-mercaptopyridine SAM. The characteristic vibrations are consistent with previous assignments $^{4}$ [47]. The average peak background emission energy is also reduced to $\hbar \omega_{\mathrm{PL}} \approx 1.83 \mathrm{eV}$, compared to the planar unpatterned gold surface.

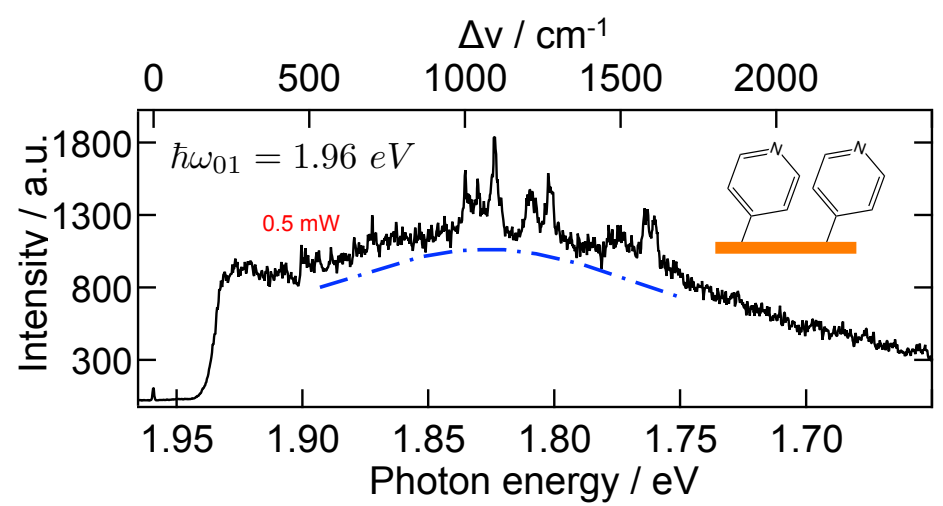

Figure 5.12. SERS spectrum from a gold nanowire array surface modified with a 4-mercaptopyridine SAM. Raman shift $\Delta v$ scale located on the top axis. A He-Ne laser source with $0.5 \mathrm{~mW}$ power and $5 \mathrm{~s}$ integration time was used for the measurement. Blue dashed-dotted line highlights the general background continuum profile.

In this case, plasmon-enhanced photoluminescence from the nanostructured gold surfaces is the primary contributor to the SERS background continuum. In both cases, the general shape of the background continuum directly corresponds to the plasmon-enhanced photoluminescence spectrum when the same excitation source is used, which is indicated with a blue dashed-dotted line (Figs. 5.10, 5.11, and 5.12). Furthermore, the peak intensity of the

\footnotetext{
${ }^{4}$ Raman vibration bands of 4-mercaptopyridine SAM modified gold nanowire array surfaces are observed at $6 \mathrm{a}\left(\mathrm{a}_{1}\right), v(\mathrm{C}-\mathrm{S})+\beta(\mathrm{C}-\mathrm{C})\left(700 \mathrm{~cm}^{-1}\right) ; 10 \mathrm{~b}\left(\mathrm{~b}_{1}\right), v(\mathrm{C}-\mathrm{S})\left(778 \mathrm{~cm}^{-1}\right) ; 1\left(\mathrm{a}_{1}\right), \beta(\mathrm{C}-\mathrm{C}-\mathrm{C})\left(1000 \mathrm{~cm}^{-1}\right) ; 18 \mathrm{a}\left(\mathrm{a}_{1}\right), \beta(\mathrm{C}-\mathrm{H})$ $\left(1036 \mathrm{~cm}^{-1}\right) ; 12\left(\mathrm{a}_{1}\right), \beta(\mathrm{C}-\mathrm{C}-\mathrm{C})+v(\mathrm{C}-\mathrm{S})\left(1094 \mathrm{~cm}^{-1}\right) ; 9 \mathrm{a}\left(\mathrm{a}_{1}\right), \beta(\mathrm{C}-\mathrm{H})\left(1210 \mathrm{~cm}^{-1}\right) ; 19 \mathrm{a}\left(\mathrm{a}_{1}\right), \gamma(\mathrm{C}=\mathrm{C} / \mathrm{C}=\mathrm{N})(1274$ $\left.\mathrm{cm}^{-1}\right) ; 8 \mathrm{~b}\left(\mathrm{~b}_{2}\right), \gamma(\mathrm{C}-\mathrm{C})\left(1577 \mathrm{~cm}^{-1}\right)$ and $8 \mathrm{a}\left(\mathrm{a}_{1}\right), v(\mathrm{C}-\mathrm{C})\left(1608 \mathrm{~cm}^{-1}\right)$.
} 
background continuum increases with increases in the excitation power, or with increases in the plasmon enhancement, consistent with plasmon-enhanced photoluminescence. From these measurements, the plasmon-enhanced photoluminescence is a strong source of the background continuum from gold surfaces. Other reports of SERS spectra accompanied by a large background continuum from gold surfaces that contained distinct peaks near $1.85 \mathrm{eV}$, however, the authors did not report photoluminescence as a source for the background continuum $[17,48]$. The importance of charge transfer between adsorbed molecules at the surface and the metal layer is acknowledged $[14,15,17,19]$, and it is concluded from these measurements that the plasmon-enhanced photoluminescence is the primary source of the background continuum and charge transfer between the adsorbed molecule and the metal layer works to modulate the continuum in intensity, emission energy location, and possibly band shape $[15,17]$.

\subsection{Conclusions}

A series of experiments were presented that demonstrate that the plasmon-modulated photoluminescence from gold nanowire array is strongly dependent on the spectral overlap of the surface plasmon resonance energy $\hbar \omega_{\text {SPR }}$ with the excitation energy $\hbar \omega_{0}$ and the interband transitions of the $5 d$ and $6 s p$ bands near the $X$ - and $L$-symmetry points of the electronic band structure. For $\hbar \omega_{01} \sim \Delta_{\mathrm{X}}$, photoluminescence involves $5 d$-6sp transitions near $\Delta_{\mathrm{X}}$, and as the spectral overlap of $\hbar \omega_{\text {SPR }}$ with $\hbar \omega_{01}$ is varied, the peak photoluminescence emission energy remains constant near $\Delta_{\mathrm{X}}\left(\hbar \omega_{\mathrm{PL}} \approx 1.87 \pm 0.01 \mathrm{eV}\right)$, while the peak photoluminescence intensity is strongly enhanced relative to the spectral overlap of $\hbar \omega_{01}$ with $\hbar \omega_{\text {SPR }}$, i.e. the intensity is strongly enhanced with a strong spectral overlap $\hbar \omega_{01} \sim \hbar \omega_{\text {SPR }}$, and weakly enhanced with a weak spectral overlap. When the excitation energy $\hbar \omega_{02}(2.33 \mathrm{eV})$ is located between $\Delta_{\mathrm{X}}$ and $\Delta_{\mathrm{L}}$, the resulting photoluminescence involves $5 d-6 s p$ transitions near both interband transitions. As the spectral overlap of $\hbar \omega_{\text {SPR }}$ with $\Delta_{\mathrm{X}}$ is varied, the peak photoluminescence energy $\hbar \omega_{\mathrm{PL}}$ shifts with $\hbar \omega_{\mathrm{SPR}}$, which is interpreted as the radiative decay of surface plasmons generated near $\Delta_{X}$, while the peak photoluminescence intensity is weakly enhanced. For a weak spectral overlap of $\hbar \omega_{\text {SPR }}$ with $\Delta_{\mathrm{X}}$, the photoluminescence characteristics resemble that of conventional photoluminescence from a planar (unpatterned) gold surface. This leads to the result that the $X$-symmetry point of the electronic band structure, which is largely negligible for conventional gold photoluminescence, is extremely important for plasmon-modulated photoluminescence. Finally, the background continuum accompanying SERS spectra from nanostructured gold surfaces (He-Ne laser source) that are modified with benzenethiol and 4- 
mercaptopyridine SAMs are shown to strongly resemble the band shapes and emission energy of plasmon-modulated photoluminescence.

\section{References}

[1] A. Mooradian, "Photoluminescence of metals," Phys. Rev. Lett. 22, 185-187, 1969.

[2] C. Chen, A. de Castro, and Y. Shen, "Surface-enhanced second-harmonic generation," Phys. Rev. Lett. 46, 145-148, 1981.

[3] C. Chen, T. Heinz, D. Ricard, and Y. Shen, "Surface-enhanced second-harmonic generation and Raman scattering," Phys. Rev. B 27, 1965-1979, 1983.

[4] M. Moskovits, "Surface-enhanced spectroscopy," Rev. Mod. Phys. 57, 783-826, 1985.

[5] G. Boyd, Z. Yu, and Y. Shen, "Photoinduced luminescence from the noble metals and its enhancement on roughened surfaces," Phys. Rev. B. 33, 7923-7936, 1986.

[6] P. Apel and R. Monreal, "Photoluminescence of noble metals," Phys. Scr. 38, 174-179, 1988.

[7] T.V. Shahbazyan, "Theory of plasmon-enhanced metal photo-luminescence," Nano Lett. 13, 194-198, 2013.

[8] M. Beversluis, A. Bouhelier, and L. Novotny, "Continuum generation from single gold nanostructures through near-field mediated intraband transitions," Phys. Rev. B 68, $115433,2003$.

[9] L. Le Thi Ngoc, M. Jin, J. Wiedemair, A. van den Berg, and E.T. Carlen, "Large area metal nanowire arrays with tunable sub-20 nm nanogaps," ACS Nano, 7, 5223-5234, 2013.

[10] M. Jin, H. van Wolferen, H. Wormeester, A. van den Berg, and E.T. Carlen, "Large-area nanogap plasmon resonator arrays for plasmonics applications," Nanoscale 4, 47124718. 2012.

[11] J. Wiedemair, L. Le, T. Ngoc, and E.T. Carlen, "Surface-enhanced Raman spectroscopy of self-assembled monolayer conformation and spatial uniformity on silver surfaces," $J$. Phys. Chem. C 118, 11857-11868, 2014.

[12] T.E. Furtak and J. Reyes, "A critical analysis of theoretical models for the giant Raman effect from adsorbed molecules," Surf. Sci. 93, 351-382, 1980.

[13] M. Moskovits, "Surface-enhanced Raman spectroscopy: a brief retrospective," J. Raman Spectrosc. 36, 485-496, 2005.

[14] J. Gersten, R. Birke, and J. Lombardi, "Theory of enhanced light scattering from molecules adsorbed at the metal-solution interface," Phys. Rev. Lett. 43, 147-150, 1979.

[15] E. Burstein, Y.J. Chen, C.Y. Chen, S. Lundquist, E. Tosatti, “'Giant' Raman scattering by adsorbed molecules on metal surfaces," Solid State Commun. 29, 567-570, 1979.

[16] H. Seki and T.J. Chuang, "The role of cavity sites in surface-enhanced Raman scatering," Chem. Phys. Lett. 100, 393-396, 1983.

[17] S. Mahajan, R.M. Cole, J.D. Speed, S.H. Pelfrey, A.E. Russell, P.N. Bartlett, S.M. Barnett, and J.J. Baumberg, "Understanding the surface-enhanced Raman spectroscopy 'background',' J. Phys. Chem. C 114, 7242-7250, 2010.

[18] J.P. Heritage, J.G. Bergman, A. Pinczuk, J.M. Worlock, "Surface picosecond Raman gain spectroscopy of a cyanide monolayer on silver," Chem. Phys. Lett. 67, 229-232, 1979.

[19] A.M. Michaels, J. Jiang, and L. Brus, "Ag nanocrystal junctions as the site for surfaceenhanced Raman scattering of single Rhodamine-6G molecules," J. Phys. Chem. B 104, 11965-11971, 2000.

[20] H. Ueba, "Effective resonant light scattering from adsorbed molecules," J. Chem. Phys. 73, 725-732, 1980.

[21] D. Shoenberg, "The Fermi surface of copper, silver and gold I. The de Haas-van Alphen Effect," Phi. Trans. R. Soc. 255, 85-133, 1962. 
[22] D. Beaglehole, "Optical properties of copper and gold in the vacuum ultra-violet," Proc. Phys. Soc. 85, 1007-1020, 1965.

[23] D. Beaglehole, "The optical properties of the noble metals," Proc. Phys. Soc. 87, 461471, 1966.

[24] N.F. Mott, The Theory of the Properties of Metals and Alloys. Clarendon Press, 1936.

[25] N. Christensen and B. Seraphin, "Relativistic band calculation and the optical properties of gold," Phys. Rev. B 4, 3321-3344, 1971.

[26] A.P. Cracknell, The Fermi Surfaces of Metals. Taylor and Francis, 1971.

[27] M. Guerrisi, R. Rosei, and P. Winsemius, "Splitting of the interband absorption edge in Au," Phys. Rev. B 12, 557-563, 1975.

[28] D. Aspnes, E. Kinsbron, and D. Bacon, "Optical properties of Au: sample effects," Phys. Rev. B 21, 3290-3299, 1980.

[29] R. Lässer, N.V. Smith, and Benbow, "Empirical band calculations of the optical properties of d-band metals," Phys. Rev. B 24, 1895-1909, 1981.

[30] A.D. Rakic, A.B. Djurisic, J.M. Elazar, and M.L. Majewski, "Optical properties of metallic films for vertical-cavity optoelectronic devices," Appl. Opt. 37, 5271-5283, 1998.

[31] P.G. Etchegoin, E.C. Le Ru, and M. Meyer, “An analytic model for the optical properties of gold," J. Chem. Phys. 125, 164705, 2006.

[32] A. Tcherniak, S. Dominguez-Medina, W.-S. Chang, P. Swanglap, L.S. Slaughter, C.F. Landes, and S. Link, "One-photon plasmon luminescence and its application to correlation spectroscopy as a probe for rotational and translational dynamics of gold nanorods," J. Phys. Chem. C 115, 15938-15949, 2011.

[33] M. Thèye, "Investigation of the optical properties of $\mathrm{Au}$ by means of thin semitransparent films," Phys. Rev. B 2, 3060-3078, 1970.

[34] K. Imura, T. Nagahara, and H. Okamoto, "Plasmon mode imaging of single gold nanorods," J. Am. Chem. Soc. 126, 12730-1, 2004.

[35] Y. Fang, W.-S. Chang, B. Willingham, P. Swanglap, S. Dominguez-Medina, and S. Link, "Plasmon emission quantum yield of single gold nanorods as a function of aspect ratio," ACS Nano 6, 7177-7184, 2012.

[36] A.H. Wilson, The Theory of Metals. Cambridge University Press, 1936.

[37] J. Leng, J. Opsal, H. Chu, M. Senko, and D.E. Aspnes, "Analytic representations of the dielectric functions of materials for device and structural modeling," Thin Solid Films 313-314, 132-136, 1998.

[38] P.B. Johnson and R.W. Christy, "Optical constants of the noble metals," Phys. Rev. B 6, 4370-4379, 1972.

[39] J.P. Wilcoxon, J.E. Martin, F. Parsapour, B. Wiedenman, and D.F. Kelley, "Photoluminescence from nanosize gold clusters," J. Chem. Phys. 108, 9137, 1998.

[40] M.B. Mohamed, V. Volkov, S. Link, and M.A. El-sayed, "The 'lightning' gold nanorods : fluorescence enhancement of over a million compared to the gold metal," Chem. Phys. Lett. 317, 517-523, 2000.

[41] E. Dulkeith, T. Niedereichholz, T. Klar, J. Feldmann, G. von Plessen, D. Gittins, K. Mayya, and F. Caruso, "Plasmon emission in photoexcited gold nanoparticles," Phys. Rev. B 70, 205424, 2004.

[42] T.V. Shahbazyan, I.E. Perakis, and J.-Y. Bigot, "Size-dependent surface plasmon dynamics in metal nanoparticles," Phys. Rev. Lett. 81, 3120-3123, 1998.

[43] M. Yorulmaz, S. Khatua, P. Zijlstra, A. Gaiduk, and M. Orrit, "Luminescence quantum yield of single gold nanorods," Nano Lett. 12, 4385-4391, 2012.

[44] H. Hu, H. Duan, J.K.W. Yang, and Z.X. Shen, "Plasmon-modulated photoluminescence of individual gold nanostructures," ACS Nano 6, 10147-10155, 2012. 
[45] E. Dulkeith, T. Niedereichholz, T. Klar, J. Feldmann, G. von Plessen, D. Gittins, K. Mayya, and F. Caruso, "Plasmon emission in photoexcited gold nanoparticles," Phys. Rev. B 70, 205424, 2004.

[46] U. Kreibig and M. Vollmer, Optical Properties of Metal Clusters. Springer-Verlag, 1995.

[47] G. Varsanyi, Vibrational Spectra of Benzene Derivates. Academic Press, 1969.

[48] M.E. Abdelsalam, P.N. Bartlett, J.J. Baumberg, S. Cintra, T.A. Kelf, and A.E. Russell, "Electrochemical SERS at a structured gold surface," Electrochem. Commun. 7, 740-744, 2005. 



\title{
Chapter 6
}

\section{Surface-enhanced Raman spectroelectrochemical analysis system $^{1}$}

\begin{abstract}
An integrated in situ surface-enhanced Raman scattering (SERS) spectroelectrochemical analysis system is presented that combines a small volume microfluidic sample chamber with a compact three-electrode configuration, which includes a gold nanowire array surface that serves dual roles as an electrochemical working electrode and as a substrate for SERS, a microfabricated platinum counter electrode, and an external $\mathrm{Ag} / \mathrm{AgCl}$ reference electrode. The nanostructured gold working electrode enables highly sensitive in situ SERS spectroscopy through large and reproducible SERS enhancements, which eliminates the need for resonant wavelength matching of the laser excitation source with the electronic absorption of the target molecule. The spectroelectrochemical analysis system provides the merits of wide applicability to target molecules, small sample volume, and low detection limit. Preliminary in situ SERS spectroelectrochemistry measurements of the metalloporphyrin hemin showing shifts of the oxidation marker band $v_{4}$ of the iron core with the nanostructured gold working electrode under potential control are presented.
\end{abstract}

\footnotetext{
${ }^{1}$ Adapted from: T. Yuan, L. Le Thi Ngoc, J. van Nieuwkasteele, M. Odijk, A. van den Berg, H. Permentier, R. Bischoff, and E.T. Carlen, "In situ surface-enhanced Raman spectroelectrochemical analysis system with a hemin modified nanostructured gold surface," Anal. Chem. 87, 2588, 2015.
} 


\subsection{Introduction to spectroelectrochemistry}

Spectroelectrochemistry combines conventional electrochemistry with spectroscopy, which provides a more complete description of chemically driven electron transfer processes and redox events. In situ Raman spectroelectrochemistry is particularly important for understanding electrode processes at the interface of a working electrode (WE) and sample solution [1]. With the WE under potential control, electrochemistry can provide thermodynamic and kinetic information of chemical processes, while in situ Raman spectroscopy provides molecular vibrational information indicative of structure and conformation. The discovery of SERS on nanostructured metal surfaces has enabled surfaceenhanced Raman spectroscopy of substances on metal surfaces, due to the large inelastic scattering cross section enhancement at the metal electrode surface, which can be used to determine structural information of very low concentrations of adsorbed molecules using Raman spectroscopy [2-4]. The combination of surface-enhanced Raman spectroscopy and electrochemistry has been shown to be a powerful tool to monitor in situ structural changes of adsorbates or reaction intermediates [5-9]. The application of spectroelectrochemistry to the investigation of redox processes is of great interest, especially when the processes can be triggered electrochemically through precise control of the WE potential.

Redox-active proteins and metalloporphyrins have been studied extensively using in situ Raman spectroelectrochemistry [10-20]. However, in most cases surface-enhanced resonance Raman scattering (SERRS), excited with high laser powers, is required in order to detect small quantities of adsorbed species on the WE $[10-12,17]$. SERRS requires that the laser source wavelength is resonant with the surface plasmon resonance wavelength of the nanostructured metal surface as well as the electronic absorption band of the molecule of interest, thus SERRS spectroelectrochemistry is most often performed on silver nanostructures. The SERRS requirement limits in situ Raman spectroelectrochemistry to molecules with absorption wavelengths in the visible spectrum, and the high laser power can affect thermally and photochemically sensitive redox systems in an uncontrolled manner. In this chapter, the design and fabrication, of an integrated in situ SERS spectroelectrochemical (SEC) analysis system is presented that combines a small volume microfluidic sample chamber with a compact three-electrode configuration, which includes a nanostructured gold surface that serves dual roles as an electrochemical working electrode and as a substrate for SERS, a microfabricated Pt counter electrode, and an external $\mathrm{Ag} / \mathrm{AgCl}$ reference electrode. Preliminary measurements of the metalloporphyrin hemin adsorbed to a 4-mercaptopyridins self-assembled monolayer shows Raman shifts of the oxidation marker band $v_{4}$ of the iron 
core in combination with redox potentials from electrochemical measurements.

\subsection{Small volume SEC system}

The process steps used to fabricate the chip for the SEC analysis system is shown in Figure 6.1. A $40 \mathrm{~nm}$ thick low stress silicon nitride ( $\mathrm{SiN}$ ) layer was first deposited onto the (100) silicon wafer by low-pressure chemical vapor deposition (Fig. 6.1(a)). Next, a positive photoresist was spin-coated on the SiN layer and patterned with conventional contact ultraviolet lithography, and subsequently developed. After the development step, a $5 \mathrm{~nm} \mathrm{Cr}$ and $100 \mathrm{~nm}$ Pt metal stack was deposited on the wafer by sputtering, and followed by a liftoff process with acetone, ultrasonication, and rinsing with deionized water (Fig. 6.1(b)). The wafer was then cut into 5 pieces (chips). There was a $1.5 \mathrm{~mm}$ separation gap at the center of the chip between the Pt electrodes where the nanostructured WE was to be fabricated. The chips were then cleaned in a 3:1 mixture of piranha $\left(\mathrm{H}_{2} \mathrm{SO}_{4}: 30 \% \mathrm{H}_{2} \mathrm{O}_{2}\right)$.

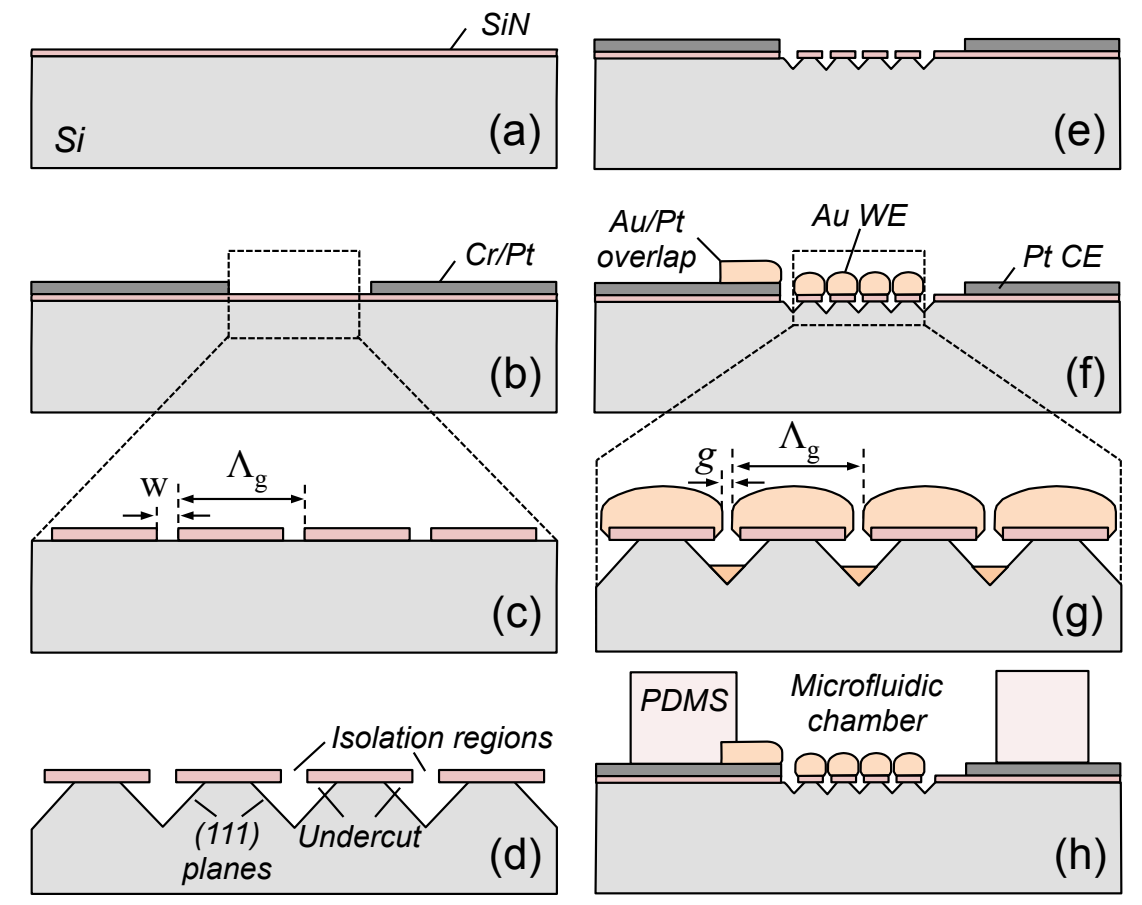

Figure 6.1. SEC chip fabrication procedure.

The nanopatterned SiN template surfaces were then formed over a $0.5 \times 0.5 \mathrm{~mm}^{2}$ at the center of the chip between the Pt electrodes (Figs. 6.1(c), 6.1(d), and 6.1(e)) using the fabrication process as described in Chapter 3. The final step of the SEC chip fabrication was the deposition of the nanostructured gold WE. A custom-made shadow mask $(0.1 \mathrm{~mm}$ thick sheet of $\mathrm{Ni}$ ) with a square hole $\left(1 \mathrm{~mm}^{2}\right)$ at its center was used to selectively deposit gold on the nanopatterned SiN region to form the nanostructured gold WE. A thin $(<1 \mathrm{~nm})$ Ti adhesion layer is deposited prior to the gold deposition to prevent delamination. A $60 \mathrm{~nm}$ gold layer was then evaporated at a rate of $0.2 \mathrm{~nm} \mathrm{~s}^{-1}$ on the SiN template with partial overlap on one of 
the Pt electrodes for external electrical connection (Figs. 6.1(f) and 6.1(g)). The microfluidic chamber was constructed from a $3 \mathrm{~mm}$ thick layer of polydimethylsiloxane (PDMS). A $7 \mathrm{~mm}$ diameter hole was punched ( $\$ 7.0 \mathrm{~mm}$ Unicore, Harris) in the PDMS layer to form a cylindrical well (volume: $115 \mathrm{~mm}^{3}$ ). The patterned PDMS layer was then bonded to the SEC chip by applying pressure (Fig. 6.1(h)). The SEC chips can be used multiple times by replacing the Ti/Au WE layer and the PDMS microfluidic chamber.

The in situ SERS SEC system consists of a SEC-cell that is optically interfaced to a Raman microscope and electrically interfaced to a potentiostat, as shown in Figure 6.2(a). The SECcell is comprised of an on-chip gold nanowire array WE and microfabricated Pt CE, directly bonded to the microfluidic sample chamber that is optically interfaced to the Raman spectrometer via a dipping microscope objective (Fig. 6.2(b)).

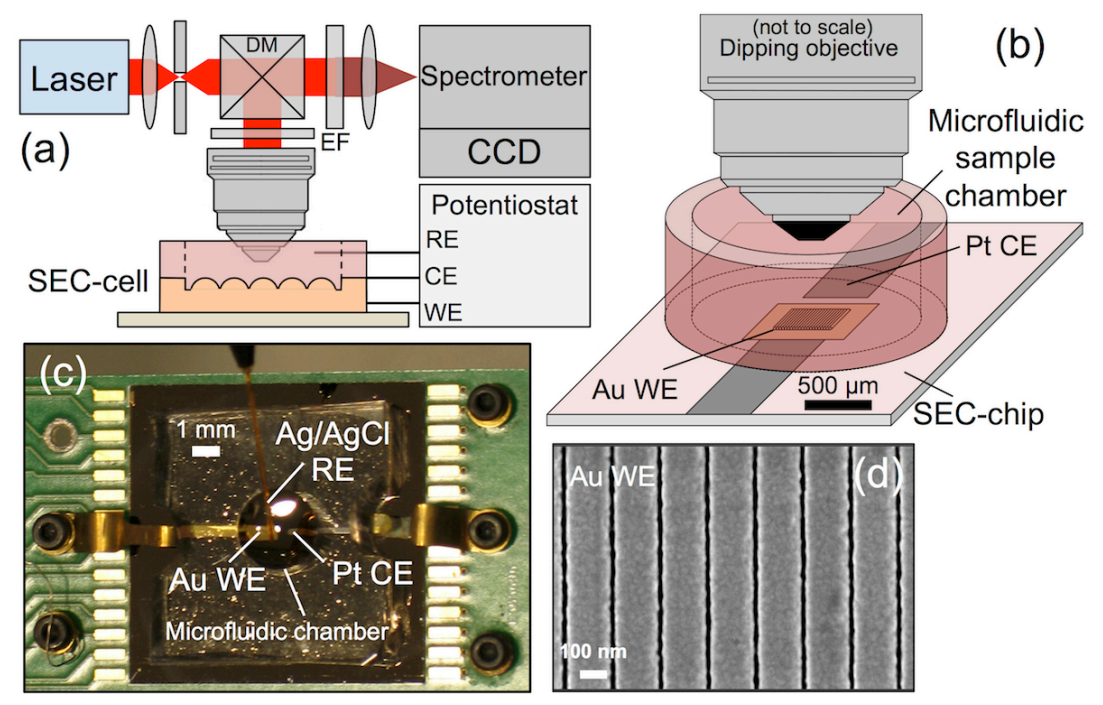

Figure 6.2. In situ SERS SEC analysis system. (a) Schematic representation of the Raman SEC analysis system, (b) SEC-cell comprised of a SEC-chip directly bonded to a small-volume microfluidic sample chamber with an optical interface to a microscope objective, (c) SEC-cell consisting of a Pt CE and a gold nanowire array WE patterned on the silicon SEC-chip, and an external $\mathrm{Ag} / \mathrm{AgCl}$ reference electrode, (d) Representative SEM image of the gold nanowire array WE.

The WE is a SERS-active gold nanowire array surface that provides extremely large and reproducible SERS enhancements. An external $\mathrm{Ag} / \mathrm{AgCl}$ reference electrode (RE) is inserted directly into the microfluidic chamber (Fig. 6.2(c)). A representative scanning electron microscopy (SEM) image of a gold nanowire array WE surface is shown in Figure 6.2(d). The peak surface plasmon resonance wavelength of the gold nanowire array WE is tuned to coincide with the laser excitation wavelength (He-Ne laser with $632.8 \mathrm{~nm}$ wavelength) to ensure an optimal SERS enhancement [20]. The SEC-chip is bonded to a custom printed circuit board and spring loaded copper clips provide electrical connection from the microfabricated Pt electrodes to the circuit board traces (Fig. 6.2(c)). The gold nanowire array WE is first modified with a 4-mercaptopyridine (MPy) self-assembled monolayer (SAM). 
Hemin is subsequently bonded via a axial coordinative bond of the heme iron to the nitrogen head-groups of the MPy SAM forming a direct molecular wire from the iron redox center to the gold WE $[14,21]$.

\subsection{SEC analysis with metalloporphyrin modified electrodes}

\subsubsection{Working electrode preparation and modification}

The gold nanowire array working electrode surface was first modified with a SAM by immersing in $1 \mathrm{mM}$ MPy in ethanol for $4 \mathrm{~h}$, followed by 1 min sonication in ethanol. After drying the MPy-modified gold electrode with $\mathrm{N}_{2}$, it was immersed in a hemin solution ( $1 \mathrm{mM}$ in DMSO) for $18 \mathrm{~h}$. Before use, the hemin modified electrode (Au/MPy/hemin) was sonicated for $1 \mathrm{~min}$ each in DMSO and water, respectively. The workflow of the preparation of the modified working electrode is displayed in Figure 6.3.

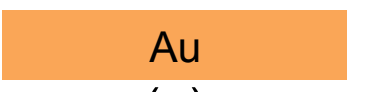

(a)

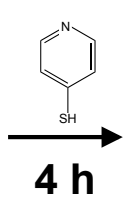

$4 \mathrm{~h}$

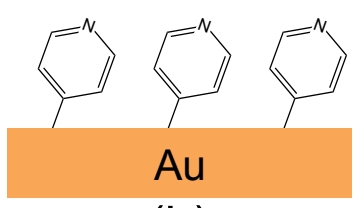

(b)

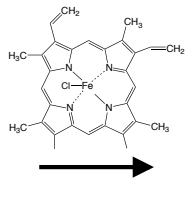

$18 \mathrm{~h}$

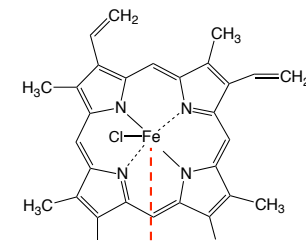

Figure 6.3. Surface preparation workflow of the MPy/hemin modified gold working electrode. (a) Clean as-deposited gold electrode surface, (b) MPy SAM formed on gold electrode, (c) Hemin axially coordinated to the MPy SAM on the working electrode.

\subsubsection{Electrochemical measurements}

All electrochemical measurements were performed with a potentiostat (VSP 200, Bio-Logic SAS, France). All potentials were measured against an $\mathrm{Ag} / \mathrm{AgCl}$ reference electrode (RE, MF2079, BaSi). $25 \mathrm{mM}$ phosphate buffered saline (PBS, pH 7.0) was used as the supporting electrolyte. The SEC-chip was clamped and bonded to a custom-made printed circuit board and spring-loaded $\mathrm{Cu}$ clips provided the electrical connection from the microfabricated $\mathrm{Pt}$ electrodes to the copper trace on the circuit board. Conventional coaxial cables and connectors were used to connect the SEC-cell to the potentiostat.

\subsubsection{Raman scattering measurements}

The surface plasmon resonance energy was tuned by controlling the pitch $\Lambda_{\mathrm{g}}$ and nanogap width $g$ (Fig. 6.1) by changing the thickness of the gold nanowire array surface layer, which was verified with reflectance measurements. For SERS measurements, the surface plasmon resonance energy was tuned near the laser excitation energy. Raman spectra of the MPy and 
MPy/hemin modified gold nanowire array surfaces were measured in PBS buffer with a laser power of $0.2 \mathrm{~mW}$. The laser power was measured at the entrance of the microscope objective. The integration time for the MPy modified WE measurements is $3 \mathrm{~s}$ and $10 \mathrm{~s}$ for the MPy/hemin modified WE. Spatial imaging was performed over a $5 \times 5 \mu \mathrm{m}^{2}$ area.

All Raman spectra were measured using a confocal Raman microscope system (alpha300R, Witech $\mathrm{GmbH}$ ), which comprises of a TE-cooled charge coupled device (DU970P-BV, Andor Technology, Belfast, Northern Ireland), UHTS300 spectrometer (f/4 300 mm FL; grating: 600 lines $\mathrm{mm}^{-1}$ ), a fiber-coupled confocal configuration with $50 \mu \mathrm{m}$ core diameter, and fiber coupled laser excitation through a $\lambda / 2$ rotator plate for polarization control and laser focusing with the microscope objective. The elastically scattered laser excitation was removed with an edge filter. A He-Ne $(1.96 \mathrm{eV} / 632.8 \mathrm{~nm})$ laser source in a backscatter configuration was focused on the surfaces using a $40 \times / 0.8$ NA dipping microscope objective (Nikon, Fluor).

\subsubsection{Results and discussions}

Electrochemical characterization. The electrical properties of the MPy/hemin modified gold nanowire WE are investigated using cyclic voltammetry $(\mathrm{CV})$. Figure 6.4 illustrates the $\mathrm{CV}$ of an MPy/hemin modified gold nanowire array WE in argon saturated PBS (pH 7.0). A welldefined, quasi-reversible redox couple ( $\mathrm{vs} \mathrm{Ag} / \mathrm{AgCl}$ ) is observed, which is characteristic of the $\mathrm{Fe}(\mathrm{III}) / \mathrm{Fe}(\mathrm{II})$ redox couple of hemin [22].

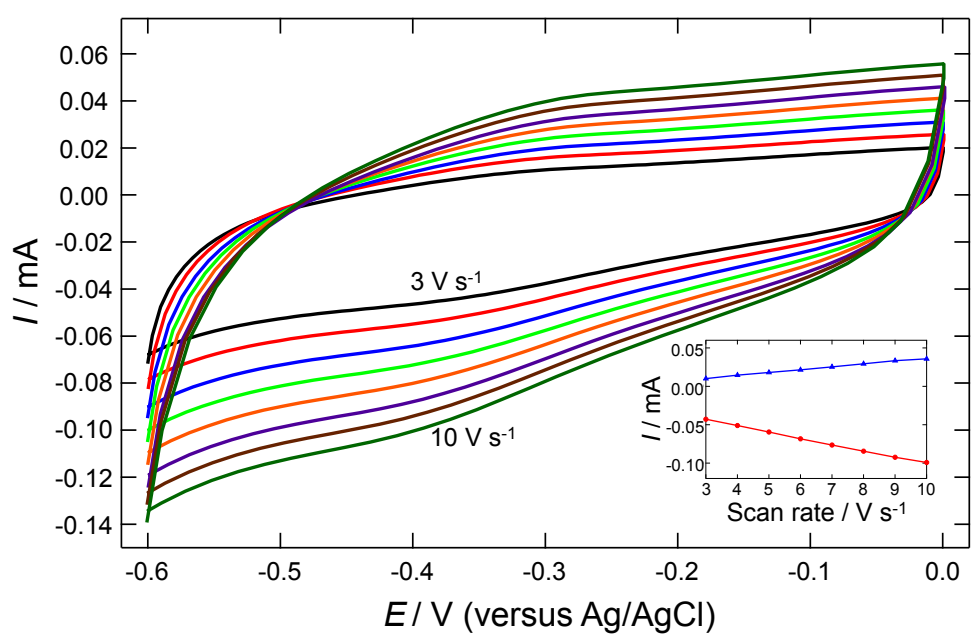

Figure 6.4. Cyclic voltammograms of an MPy/hemin modified gold nanowire array WE in $25 \mathrm{mM}$ phosphate buffer saline (PBS, $\mathrm{pH} 7.0$, nitrogen saturated) with scan rates ranging from 3 to $10 \mathrm{~V} \mathrm{~s}^{-1}$ in $1 \mathrm{~V} \mathrm{~s}^{-1}$ increments. Insert: plots of redox peak currents versus scan rate, blue trace: oxidation peaks and red trace: reduction peaks.

As shown in the insert of Fig. 6.4, the anodic and cathodic peak currents increase linearly over scan rate in the range of $3-10 \mathrm{~V} \mathrm{~s}^{-1}$, thus indicating that the redox reaction of the immobilized hemin is a surface-controlled process [23], which further supports successful 
immobilization of hemin on the gold nanowire WE. The CV characteristics of MPy/hemin modified gold nanowire WE are similar with those observed from the gold disk electrode (1.6 $\mathrm{mm}$ diameter, Bioanalytical System) using a similar surface modification procedure. The surface coverage of hemin on the gold nanowire electrode is $\Gamma \approx 9 \times 10^{-12} \mathrm{~mol} \mathrm{~cm}^{-2}$, using $\Gamma=Q(n F A)^{-1}$, where $Q$ is the integrated charge across the reduction peak, $n$ is the number of electrons in a faradic reaction, $F$ is Faraday's constant, and $A$ is the surface area of the electrode.

In situ SERS measurements. The SERS spectra measured from the MPy/hemin modified gold nanowire array WE using the new in situ SERS SEC analysis system are shown in Figure 6.5. Figure 6.5(a) shows a schematic of the MPy/hemin modified gold WE. The SERS vibration bands of the MPy modified gold nanowire array WE are shown in the spectrum of Figure 6.5(b), which are observed at $6 \mathrm{a}\left(\mathrm{a}_{1}\right), \mathrm{v}(\mathrm{C}-\mathrm{S})+\beta(\mathrm{C}-\mathrm{C})\left(700 \mathrm{~cm}^{-1}\right) ; 10 \mathrm{~b}\left(\mathrm{~b}_{1}\right), \gamma(\mathrm{C}-\mathrm{H})$ $\left(778 \mathrm{~cm}^{-1}\right) ; 1\left(\mathrm{a}_{1}\right), \beta(\mathrm{C}-\mathrm{C}-\mathrm{C})\left(1000 \mathrm{~cm}^{-1}\right) ; 18 \mathrm{a}\left(\mathrm{a}_{1}\right), \beta(\mathrm{C}-\mathrm{H})\left(1036 \mathrm{~cm}^{-1}\right) ; 12\left(\mathrm{a}_{1}\right), \beta(\mathrm{C}-\mathrm{C}-\mathrm{C})+$ $v(\mathrm{C}-\mathrm{S})\left(1094 \mathrm{~cm}^{-1}\right) ; 9 \mathrm{a}\left(\mathrm{a}_{1}\right), \beta(\mathrm{C}-\mathrm{H})\left(1210 \mathrm{~cm}^{-1}\right) ; 3\left(\mathrm{~b}_{2}\right), \beta(\mathrm{C}-\mathrm{H})\left(1274 \mathrm{~cm}^{-1}\right) ; 19 \mathrm{a}\left(\mathrm{a}_{1}\right)$, $v(\mathrm{C}=\mathrm{C} / \mathrm{C}=\mathrm{N})\left(1492 \mathrm{~cm}^{-1}\right) ; 8 \mathrm{~b}\left(\mathrm{~b}_{2}\right), v(\mathrm{C}-\mathrm{C})\left(1577 \mathrm{~cm}^{-1}\right)$; and $8 \mathrm{a}\left(\mathrm{a}_{1}\right), v(\mathrm{C}-\mathrm{C})\left(1608 \mathrm{~cm}^{-1}\right)$, and are consistent with previous assignments [24].
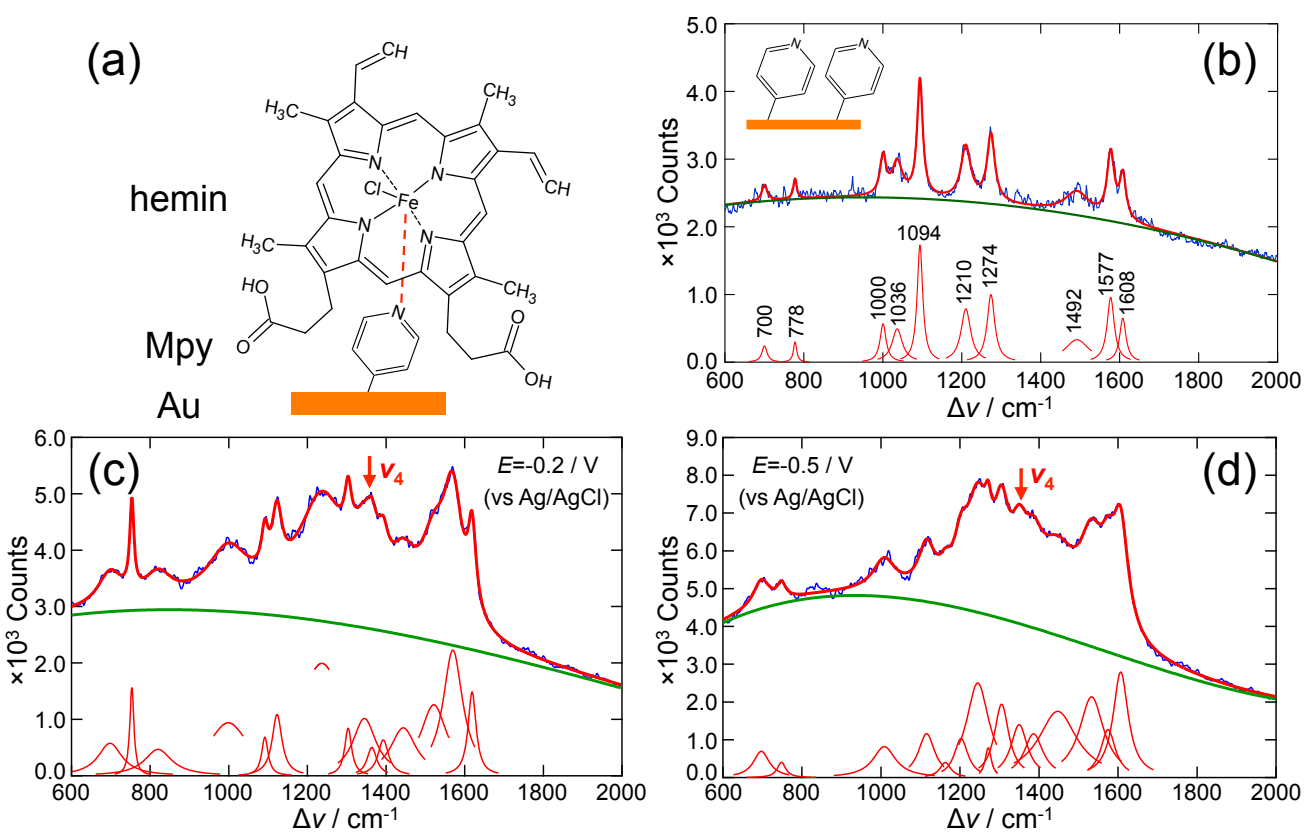

Figure 6.5. SERS spectra of a gold nanowire array WE under potential control in $25 \mathrm{mM}$ sodium phosphate buffer. (a) Schematic of the MPy/hemin modified gold surface, (b) SERS spectrum of MPy modified gold surface, (c) SERS spectrum of MPy/hemin modified gold surface with $E=-0.2 \mathrm{~V}$, (d) SERS spectrum of MPy/hemin modified gold surface with $E=-0.5 \mathrm{~V}$. Blue traces: raw data, red traces: modeled data, and green traces: modeled background (the vibration bands are modeled with a Lorentzian band shape after subtraction of the background using a cubic polynomial function).

The labels $\gamma, \beta$, and $v$ indicate out-of-plane bending, in-plane bending, and stretching modes, respectively. The spatially averaged enhancement factor is in the range of $10^{7}-10^{8}$, which has 
been characterized on gold nanowire array surfaces [20]. The signal-to-noise ratio (SNR) is defined as $S N R=\bar{S} / \sigma$, where $\bar{S}$ is the average band intensity and $\sigma$ is the amplitude of the noise signal in the region of the band [25]. For the $1\left(\mathrm{a}_{1}\right)$ vibration mode, $S N R_{1(\mathrm{a} 1)} \approx 12$, and for the $12\left(\mathrm{a}_{1}\right)$ vibration mode $S N R_{12(\mathrm{a} 1)} \approx 22$.

The SERS spectra measured from the MPy/hemin modified gold nanowire array WE are shown in Figures 6.5(c), 6.5(d) and 6.6. Since the primary scope of this chapter is the development of the SEC analysis system, a preliminary analysis of the measured SERS spectra of the MPy/hemin modified WE is presented. Figures 6.5(c) and 6.5(d) depict SERS spectra for WE potentials $E=-0.2 \mathrm{~V}$ and $E=-0.5 \mathrm{~V}$ (vs. $\mathrm{Ag} / \mathrm{AgCl}$ ), respectively. Many of the MPy vibration bands are observed in the SERS spectra, such as $700 \mathrm{~cm}^{-1}\left(6 \mathrm{a}\left(\mathrm{a}_{1}\right)\right), 1000 \mathrm{~cm}^{-1}$ $\left(1\left(a_{1}\right)\right)$ and $1094 \mathrm{~cm}^{-1}\left(12\left(a_{1}\right)\right)$. Certain vibrational modes of the porphyrin ring of hemin have been observed to shift in frequency consistent with the oxidation state, axial ligation, or coordination, and spin state of the central iron atom [11-15, 26, 27].
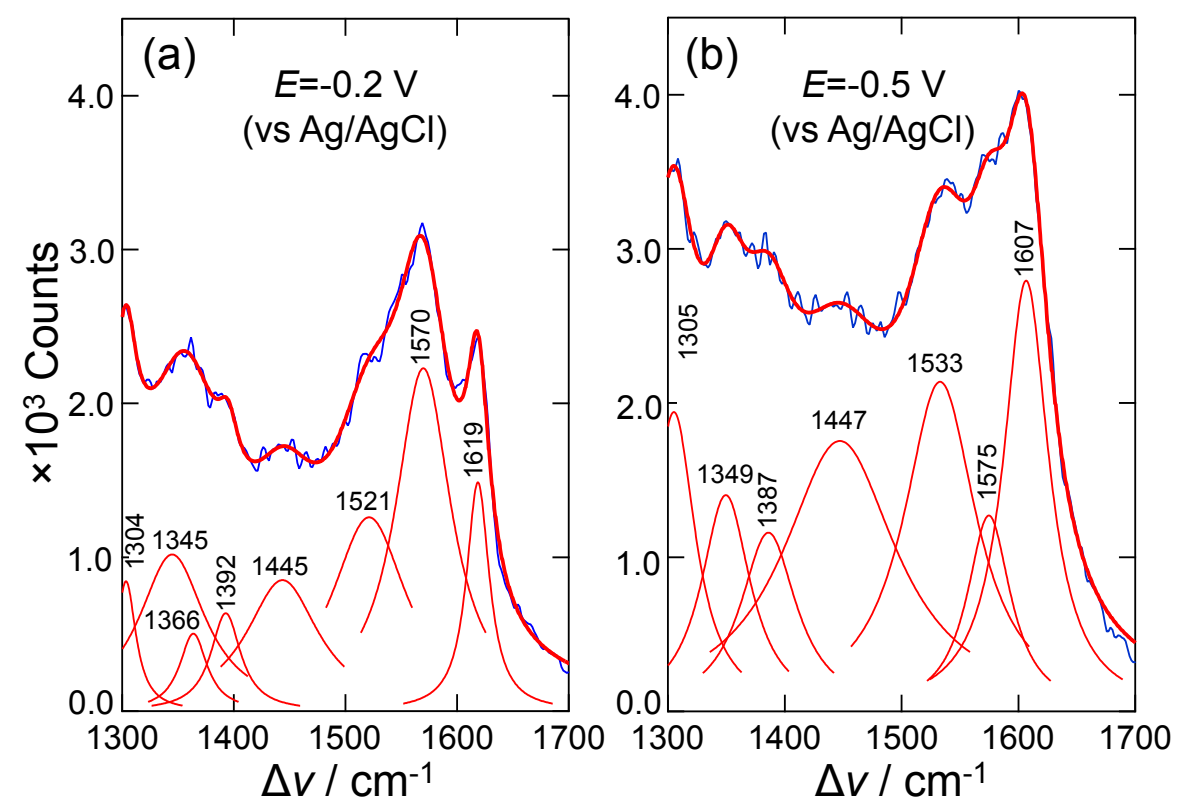

Figure 6.6. SERS spectra from MPy/hemin modified gold nanowire array WE in the marker band region (background corrected). (a) Electrode potential $E=-0.2 \mathrm{~V}$, (b) Electrode potential $E=-0.5 \mathrm{~V}$. Blue traces: raw data and red traces: modeled data.

The $v(\mathrm{C}-\mathrm{C})$ and $v(\mathrm{C}-\mathrm{N})$ stretch vibrations of the porphyrin ring are typically observed to shift in frequency according to the iron oxidation state, which occur in the marker band range of $1300-1700 \mathrm{~cm}^{-1}$. The $v_{4}\left(\mathrm{~A}_{1 \mathrm{~g}}\right)$ vibration mode is due mainly to $\mathrm{C}-\mathrm{N}$ stretch vibrations of the pyrrole subunits that are sensitive to electron transfer in the $\pi^{*}$ orbital of the porphyrin ring and is an indicator of the iron oxidation state [28]. The $v_{4}$ mode is observed in the frequency range of $1368-1377 \mathrm{~cm}^{-1}$ for ferric $\mathrm{Fe}(\mathrm{III})$ hemin and in the range of $1344-1364 \mathrm{~cm}^{-1}$ for ferrous $\mathrm{Fe}(\mathrm{II})$ hemin [18,29]. 
Figure 6.6 shows the corresponding SERS spectra in the marker band range. For an applied potential of $E=-0.2 \mathrm{~V}$ (Figure 6.6(a)), two bands can be resolved at $1345 \mathrm{~cm}^{-1}$ and $1366 \mathrm{~cm}^{-1}$. The $v_{4-1}=1345 \mathrm{~cm}^{-1}$ band lies in the ferrous state range with $81 \%$ of the integrated counts, and the $v_{4-2}=1366 \mathrm{~cm}^{-1}$ band lies in the ferric state range with $19 \%$ of the integrated counts, which indicates the partial oxidation of the iron cores on the surface [18]. For an applied potential of $E=-0.5 \mathrm{~V}$ (Fig. 6.6(b)), a single vibration band is observed at $v_{4} \approx 1349 \mathrm{~cm}^{-1}$, which lies in the reduced ferrous state range, thus indicating reduction of the iron cores on the surface.

The vibration modes $v_{2}\left(\mathrm{~A}_{1 \mathrm{~g}}\right), v_{3}\left(\mathrm{~A}_{1 \mathrm{~g}}\right)$, and $v_{10}\left(\mathrm{~B}_{1 \mathrm{~g}}\right)$, which are mainly due to the $v(\mathrm{C}-\mathrm{C})$ stretch vibrations, are typical indicators of the coordination and spin state of hemin. The bands at $1570 \mathrm{~cm}^{-1}$ (Fig. 6.6(a), $E=-0.2 \mathrm{~V}$ ) and $1574 \mathrm{~cm}^{-1}$ (Fig. 6.6(b), $E=-0.5 \mathrm{~V}$ ) are most likely associated with the $v_{2}$ mode of hemin, which correspond well to previous assignments of five-coordinated ferric heme with high spin state (5cHS) [30]. The bands at $1619 \mathrm{~cm}^{-1}$ (Fig. 6.6(a), $E=-0.2 \mathrm{~V}$ ) and $1607 \mathrm{~cm}^{-1}$ (Fig. 6.6(b), $E=-0.5 \mathrm{~V}$ ) are likely associated with the $v_{10}\left(B_{1 \mathrm{~g}}\right)$ mode of ferrous hemin, which also correspond well to previous assignments of $5 \mathrm{cHS}$ heme [30]. It is important to note that the $v_{2}$ and $v_{10}$ modes overlap the MPy ring stretch $v(\mathrm{C}$ C) modes $8 \mathrm{~b}\left(\mathrm{~b}_{2}\right)$ at $1577 \mathrm{~cm}^{-1}$ and $8 \mathrm{a}\left(\mathrm{a}_{1}\right)$ at $1608 \mathrm{~cm}^{-1}$ (Fig. 6.5(b)), respectively. The broad vibration modes at $1445 \mathrm{~cm}^{-1}$ (Fig. 6.6(a), E=-0.2 V) and $1447 \mathrm{~cm}^{-1}$ (Fig. 6.6(a), $E=-0.2 \mathrm{~V}$ ) may be due to a combination of the in-plane ring stretching mode of MPy $19 b\left(b_{2}\right)$ near 1450 $\mathrm{cm}^{-1}$ [24], and the $v_{3}(\mathrm{~A} 1 \mathrm{~g})$ mode near $1495 \mathrm{~cm}^{-1}$ of hemin, but requires further investigation before definitive assignment [30]. The bands at $1392 \mathrm{~cm}^{-1}$ and $1387 \mathrm{~cm}^{-1}$ are not currently assigned, but were previously observed in SERRS spectra of hemin adsorbed on silver surfaces in the presence of pyridine [31]. It is important to note that these observations and assignments are preliminary. From the estimation of the hemin surface density from the electrochemical measurements, approximately 50,000 hemin molecules on average are in the collection volume of the SERS measurement. Based on the SNR of the measurement, the limit of detection is in the range of 2,000-5,000 hemin molecules.

\subsection{Conclusions}

A new in situ SERS spectroelectrochemical analysis platform was presented that is comprised of a small volume sample chamber and a gold nanowire working electrode surface, which allows for simultaneous SERS and electrochemical investigation of any modified surface with low laser power. The gold nanowire array surface has a large and reproducible SERS enhancement, which enables highly sensitive surface-enhanced Raman spectroscopy without resonant excitation of the molecule of interest, thus simplifying the measurement apparatus 
and expanding the applicability to a wider range of target molecules. In addition, the small volume microfluidic chamber is especially suitable for the analysis of hazardous or costly samples.

\section{References}

[1]W. Pleith, G.S. Wilson, and C. Gutiérrez De La Fe, "Spectroelectro-chemistry: A survey of in situ spectroscopic techniques," Pure Appl. Chem. 70, 1395-1414, 1998.

[2]M. Fleischmann, P.J. Hendra, and A.J. McQuillan, "Raman spectra of pyridine adsorbed at a ailver electrode," Chem. Phys. Lett. 26, 163-166, 1974.

[3]M. Moskovits, "Surface-enhanced spectroscopy," Rev. Mod. Phys. 57, 783-826, 1985.

[4]S. Nie and S. Emory, "Probing single molecules and single nanoparticles by surfaceenhanced Raman scattering," Science 275, 1102-1106, 1997.

[5]I.C.G. Thanos, "An In-Situ Raman Spectroscopic study of reduction of $\mathrm{HNO}_{3}$ on a rotating silver electrode," J. Electroanal. Chem. 200, 231-241, 1986.

[6]T. Itoh, T. Maeda, and A. Kasuya, "In situ surface-enhanced Raman scattering spectroelectrochemistry of oxygen species," Faraday Discuss. 132, 95, 2006.

[7]D.-Y. Wu, J.-F. Li, B. Ren, and Z.-Q. Tian, "Electrochemical surface-enhanced Raman spectroscopy of nanostructures," Chem. Soc. Rev. 37, 1025-1041, 2008.

[8]K.S. Joya and H.J.M. de Groot, "Electrochemical in situ surface enhanced Raman spectroscopic characterization of a trinuclear ruthenium complex, Ru-red," J. Raman Spectrosc. 44, 1195-1199, 2013.

[9]S. Hy, Y.-H. Chen, J. Liu, J. Rick, and B.-J. Hwang, "In situ surface enhanced Raman spectroscopic studies of solid electrolyte interphase formation in lithium ion battery electrodes," J. Power Sources 256, 324-328, 2014.

[10] W.T. Borden and E.R. Davidson, "Surface-enhanced resonance Raman scattering from cytochrome-c and myoglobin adsorded on a silver electrode," J. Am. Chem. Soc. 102, 7960-7962, 1980.

[11] L.A. Sanchez and T.G. Spiro, "Surface-enhanced Raman spectroscopy as a monitor of iron(III) protoporphyrin reduction at a silver electrode in aqueous and acetonitride solutions: Vibronic resonance enhancement amplified by surface enhacement," J. Phys. Chem. 89, 763-768, 1985.

[12] P. Hildebrandt and M. Stockburger, "Cytochrome c at charged interfaces. 1. Conformational and redox equilibria at the electrode/ electrolyte interface probed by surface-enhanced resonance Raman spectroscopy," Biochemistry, 28, 6710-6721, 1989.

[13] S. Lecomte, H. Wackerbarth, T. Soulimane, G. Buse, and P. Hildebrandt, "Time-resolved surface-enhanced resonance Raman spectroscopy for studying electron-transfer dynamics of heme proteins," J. Am. Chem. Soc, 7863, 7381-7382, 1998.

[14] D.H. Murgida, P. Hildebrandt, D. Berlin, J. Wei, Y. He, H. Liu, and D.H. Waldeck, "Surface-enhanced resonance Raman spectroscopic and electrochemical study of cytochrome c bound on electrodes through coordination with pyridinyl-terminated selfassembled monolayers," J. Phys. Chem. B 108, 2261-2269, 2004.

[15] D. Millo, A. Bonifacio, A. Ranieri, M. Borsari, C. Gooijer, and G. van der Zwan, "Voltammetric and surface-enhanced resonance Raman spectroscopic characterization of cytochrome-c adsorbed on a 4-mercaptopyridine monolayer on silver electrodes," Langmuir 23, 4340-4345, 2007.

[16] M. Grosserueschkamp, C. Nowak, and W. Knoll, "Time-resolved surface-enhanced resonance Raman spectroelectrochemistry of heme proteins," Spectroscopy 24, 125-129, 2010. 
[17] K. Sengupta, S. Chatterjee, S. Samanta, and A. Dey, "Direct observation of intermediates formed during steady-state electrocatalytic $\mathrm{O}_{2}$ reduction by iron porphyrins," Proc. Natl. Acad. Sci. USA 110, 8431-8436, 2013.

[18] Y. Wang, P.C. Sevinc, Y. He, and H.P. Lu, "Probing ground-state single-electron selfexchange across a molecule-metal interface," J. Am. Chem. Soc. 133, 6989-6996, 2011.

[19] W.-B. Cai, I. C. Stefan, and D. a Scherson, "Determination of adsorption isotherm of species adsorbed on roughened silver electrodes from in situ quantitative surface enhanced Raman spectroscopy," J. Electroanal. Chem. 524-525, 36-42, 2002.

[20] L. Le Thi Ngoc, M. Jin, J. Wiedemair, A. van den Berg, and E.T. Carlen, "Large area metal manowire arrays with tunable sub-20 nm nanogaps," ACS Nano 7, 5223-5234, 2013.

[21] J. Wei, H. Liu, A.R. Dick, H. Yamamoto, Y. He, and D.H. Waldeck, "Direct wiring of cytochrome c's heme unit to an electrode: electrochemical studies," J. Am. Chem. Soc. 124, 9591-9599, 2002.

[22] A.K. Udit, M.G. Hill, V.G. Bittner, F. H. Arnold, and H. B. Gray, "Reduction of dioxygen catalyzed by pyrene-wired heme domain cytochrome P450 BM3 electrodes," $J$. Am. Chem. Soc. 126, 10218-10219, 2004.

[23] A.J. Bard and L. R. Faulkner, Electrochemical Methods: Fundamentals and Applications. 2nd Ed., John Wiley \& Sons, 2001.

[24] G. Varsanyi, Vibrational Spectra of Benzene Derivates. Academic Press, 1969.

[25] R.L. McCreery, Raman Spectroscopy for Chemical Analysis. John Wiley \& Sons, 2000.

[26] T.G. Spiro and T.C. Strekas, "Resonance Raman spectra of heme proteins. Effects of oxidation and spin state," J. Am. Chem. Soc. 570, 338-345, 1974.

[27] S. Choi, T.G. Spiro, K.C. Langry, and K.M. Smith, "Vinyl influences on protoheme resonance Raman spectra: Nickel(II) protoporphyrin IX with deuterated vinyl groups," $J$. Am. Chem. Soc. 104, 4337-4344, 1982.

[28] T. Kitagawa and Y. Mizutani, "Resonance Raman spectra f highly oxidized metalloporphyrins and heme proteins," Coord. Chem. Rev. 135, 685-735, 1994.

[29] M. Feng and H. Tachikawa, "Surface-enhanced resonance Raman spectroscopic characterization of the protein native structure," J. Am. Chem. Soc. 130, 7443-7448, 2008.

[30] S. Oellerich, H. Wackerbarth, and P. Hildebrandt, "Spectroscopic characterization of non-native conformational states of cytochrome c," J. Phys. Chem. B 106, 6566-6580, 2002.

[31] J.J. McMahon, S. Baer, and C.A. Melendres, "Surface Raman scattering and electrochemistry of iron protoporphyrin IX at a polycrystalline silver electrode," J. Phys. Chem. 90, 1572-1577, 1986. 



\section{Chapter 7}

\section{Conclusions and Recommendations}

\subsection{Conclusions}

In this thesis, a new fabrication technology is presented that combines conventional and well characterized top-down microfabrication steps with nanofabrication steps to realize a template surface that facilitates the growth of the physical vapor deposited metal layer on the template surface, which reduces the metal growth rate in the lateral direction between adjacent template surfaces, and allows for precise control of the separation nanogaps between adjacent nanowires that are electrically isolated, as shown in Figure 7.1. The advantage of this new fabrication technology is that extremely dense arrays of electrically isolated metal nanowires that have controllable elliptical surface shapes and sub-20 nm separation nanogaps can be realized without the use of a metal etching or milling step.
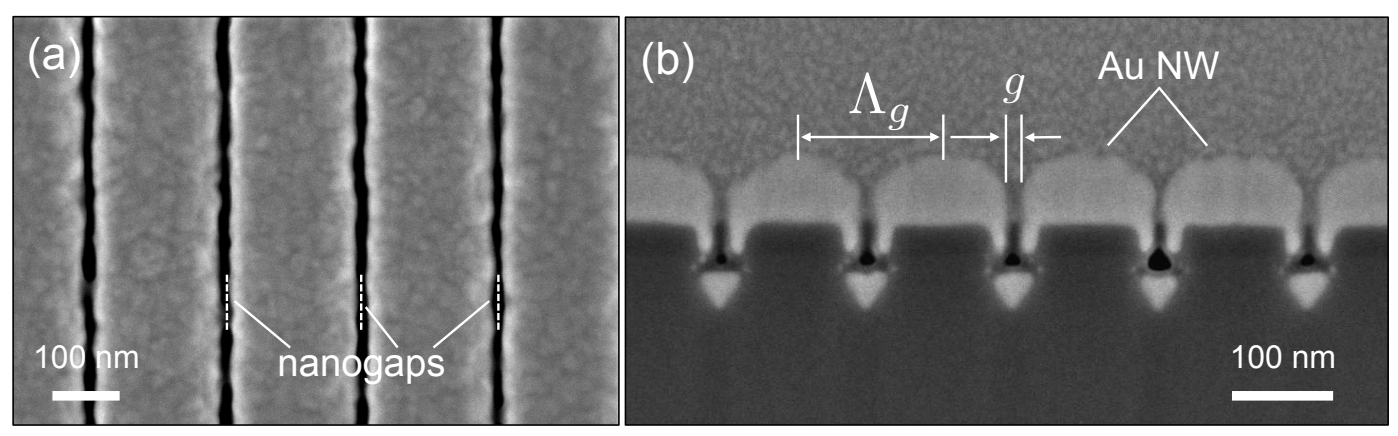

Figure 7.1. Representative SEM images of gold nanowire array surface with pitch $\Lambda_{\mathrm{g}}=200 \mathrm{~nm}$ and nanogap spacing $g=10 \mathrm{~nm}$. (a) Top view, (b) Cross section view.

Furthermore, the metal nanowire array surfaces are reusable by simply replacing the metal layer, which does not damage the template substrate materials or alter the template dimensions. This simple fabrication method can provide very uniform and high density $\left(\sim 10^{5}\right.$ nanowires $\mathrm{cm}^{-1}$ ) metal nanowire arrays with small diameters (lower limit: approximately 50 $\mathrm{nm}$ ), small separation nanogaps (lower limit: approximately $5 \mathrm{~nm}$ ), large length to pitch ratio of more than approximately $10^{4}$, and extremely large nanogap length density on the order of 1 $\mathrm{km} \mathrm{cm}^{-2}$ without the use of etching or milling the metal layer. 


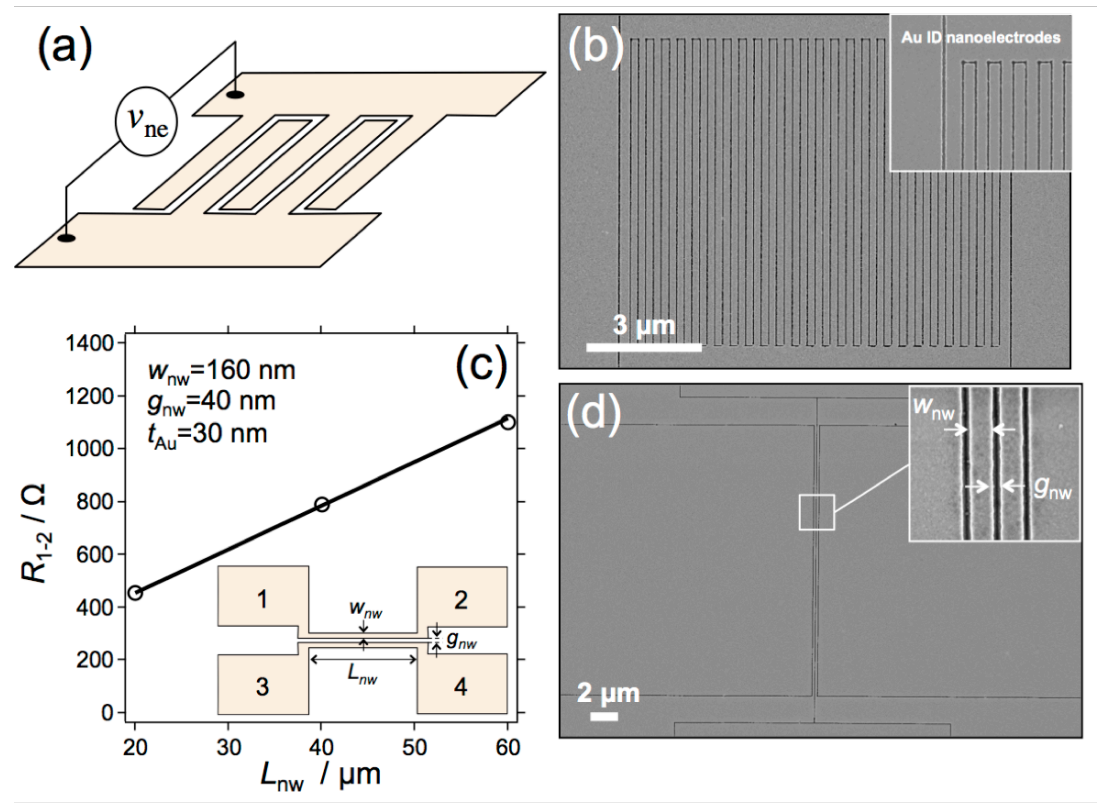

Figure 7.2. Interdigitated metal nanoelectrode substrate. (a) Schematic of 4-nanogap electrode configuration, (b) SEM image of 49-nanogap gold nanoelectrode device, (c) Measured electrical resistances $R_{1-2}$ of the gold nanowire test structure shown in the inset image, (d) SEM image of the test structure.

The metal nanowire array surfaces can be patterned into functional interdigitated nanoelectrode arrays with the number of nanogaps ranging from one up to thousands. Figure 7.2 shows a test that demonstrates electrically isolated nanowires arrays used as an electrode or interdigitated nanoelectrode; $v_{\text {ne }}$ provides a controllable electric field across each of the nanogaps. This configuration can be used for a variety of applications, such as the study of molecular conductance from molecules that bridge the nanogap width, electrochemical nanosensors, and combined electrochemical and surface spectroscopy.

The metal nanowire array surfaces are well suited for plasmonics applications, which use metal nanostructures that couple far-field electromagnetic radiation into electromagnetic surface modes, called surface plasmon polaritons, that are confined to the interface of a nonabsorbing dielectric, with positive real dielectric constant, to a conductive material with negative real value of its dielectric function, which is typically a noble metal. When two metal nanoparticles are in close proximity with the enhanced electromagnetic fields around each nanostructure coherently interfere, thus resulting in a coupled-plasmon electromagnetic field across the nanogap, which results in an increased in the electromagnetic field enhancement when the excitation polarization is aligned perpendicular to the nanogap length. For small nanogaps, below about $20 \mathrm{~nm}$, the electromagnetic field enhancement in the nanogap increases rapidly. Figure 7.3 shows an example of the confined and enhanced electromagnetic field concentrated in the nanogap region between two nanowire structures with cross section geometry modeled after the SEM images shown in Figure 7.1(b). 


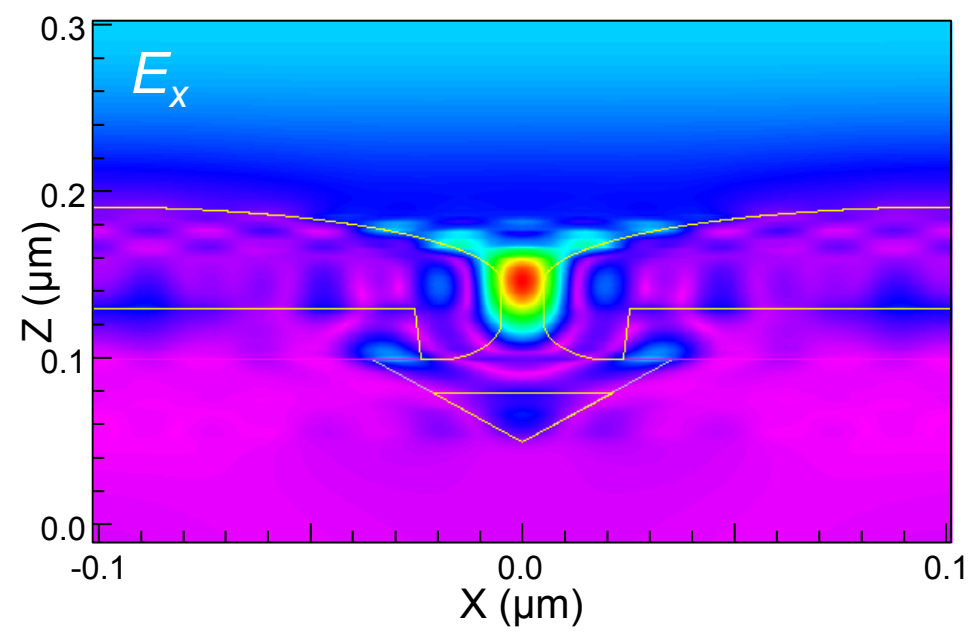

Figure 7.3. Electromagnetic field enhancement in the nanogap separation between adjacent gold nanowire structures calculated using rigorous waveguide coupled analysis software.

Reflection spectroscopy has been used to demonstrate the plasmonic functionality, and tunability and uniformity of the dimensions of the metal-NW arrays, pitch and nanogap, with a normally incident linearly polarized white light source that is aligned perpendicular to the nanogaps. The reflectance measurements of gold and silver nanowire array surfaces demonstrated that the localized surface plasmon resonance wavelength could be tuned to excitation laser wavelengths $532 \mathrm{~nm}, 633 \mathrm{~nm}$, and $785 \mathrm{~nm}$. In all cases, the localized surface plasmon resonance wavelength red-shifted when the nanogap spacing decreased, thus demonstrating the strong dependence on the couple-resonance mode in the nanogap.
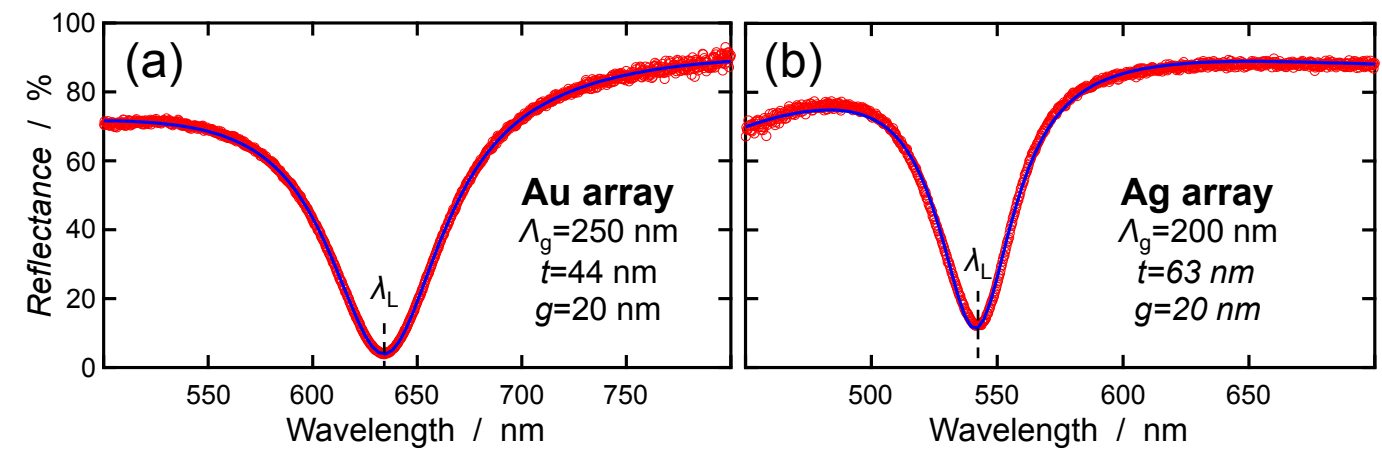

Figure 7.4. Measured reflectance spectra of (a) Gold and, (b) Silver nanowire array surfaces.

The localized surface plasmon resonance can be assessed with the resonance quality factor $Q$, which is a dimensionless metric that represents the ratio of energy stored in the resonator to energy loss per cycle. The experimental quality factor can be estimated with $Q \approx \lambda_{\mathrm{L}} / \Delta \lambda$, where $\lambda_{\mathrm{L}}$ is the localized surface plasmon resonance wavelength and $\Delta \lambda$ is the full-width halfmaximum of the localized surface plasmon resonance band, which are both measured from the measured reflectance spectrum. Figure 7.4 shows reflectance spectra for gold and silver nanowire array surfaces for a particular nanowire pitch $\Lambda_{\mathrm{g}}$, metal thickness $t$, and nanogap spacing $g$. For the gold nanowire array surfaces, $Q \approx 10$ (Fig. 7.4(a)), and for the silver 
nanowire array surfaces $Q \approx 15$ (Fig. 7.4(b)). The relatively low experimental $Q$-factors are typical for noble metals, such as gold and silver, due to absorptive damping in the metal; however, some inhomogeneous broadening of the resonance dip occurs to the finite surface roughness of the polycrystalline metal layer.

The spatially averaged SERS enhancement factor of gold nanowire array surfaces was assess from measurements of the ring-breathing mode of benzenethiol chemisorbed in the nanowire surface and was found to be in the range of $10^{7}-10^{8}$, which is extremely large considering that all of the benzenethiol molecules in the self-assembled have been included in the estimation of the enhancement factor, while it is well-known that the molecules in the nanogap region generate the majority of the measured signal. The standard deviation of the spatially averaged SERS enhancement factor is on the order of $10^{6}$, which represents an $11 \%$ enhancement factor variation.

Conventional photoluminescence from planar (un-patterned) as-deposited thin films of polycrystalline gold and plasmon-modulated photo-luminescence from large area nanostructured gold nanogap surfaces were investigated. In particular, the high uniformity of the nanostructured gold surfaces used in this thesis have tunable and highly reproducible plasmon resonances, thus enabling such systematic studies. A series of experiments have demonstrated that the plasmon-modulated photoluminescence from gold nanostructures is strongly dependent on the spectral overlap of the surface plasmon resonance energy with the excitation energy and the interband transition of the $5 d$ and $6 s p$ bands near the $X$-symmetry point of the electronic band structure.

For the He-Ne laser, with an emission energy of $1.96 \mathrm{eV}$, which is close to $\Delta_{\mathrm{X}}$, of gold, the photoluminescence involves $5 d-6 s p$ transitions near $\Delta_{\mathrm{X}}$, and as the spectral overlap of localized surface plasmon resonance with the laser energy is varied, the peak photoluminescence emission energy remains approximately constant near $\Delta_{\mathrm{X}}$ $\left(\hbar \omega_{\mathrm{PL}} \approx 1.87 \pm 0.01 \mathrm{eV}\right.$ ), while the peak photoluminescence intensity is strongly enhanced relative to the spectral overlap of laser with the localized surface plasmon resonance band, i.e. the intensity is strongly enhanced with a strong spectral overlap of the laser source and the localized surface plasmon resonance band, and weakly enhanced with a weak spectral overlap.

For the Nd-YAG laser, with excitation energy of $2.33 \mathrm{eV}$, is located near the interband transition $\Delta_{\mathrm{X}}$, the resulting photoluminescence involves $5 d-6 s p$ transitions near both interband transitions. As the spectral overlap of localized surface plasmon resonance band with $\Delta_{\mathrm{X}}$ is varied, the peak photoluminescence energy $\hbar \omega_{\mathrm{PL}}$ shifts with localized surface plasmon 
resonance band, which is interpreted as the radiative decay of surface plasmons generated near $\Delta_{\mathrm{X}}$, while the peak photoluminescence intensity is weakly enhanced. For a weak spectral overlap of the localized surface plasmon resonance band with $\Delta_{\mathrm{X}}$, the photoluminescence characteristics resemble that of conventional photoluminescence from a planar gold surface. This leads to the result that the $X$-symmetry point of the electronic band structure, which is largely negligible for conventional gold photo-luminescence, is extremely important for plasmon-modulated photo-luminescence. The background continuum accompanying surfaceenhanced Raman scattering (SERS) spectra, from gold nanowire array surfaces (He-Ne laser) modified with benzenethiol and 4-mercaptopyridine SAMs, was found to strongly correlate to plasmon-modulated photoluminescence, as shown in Figure 7.5. Using near-infrared excitation sources, which is lower than the interband transitions of gold, can minimize the occurrence of the strong background continuum of gold plasmonic surfaces, due to plasmonenhanced photo-luminescence, as well as the carbon contamination of silver plasmonic surfaces.

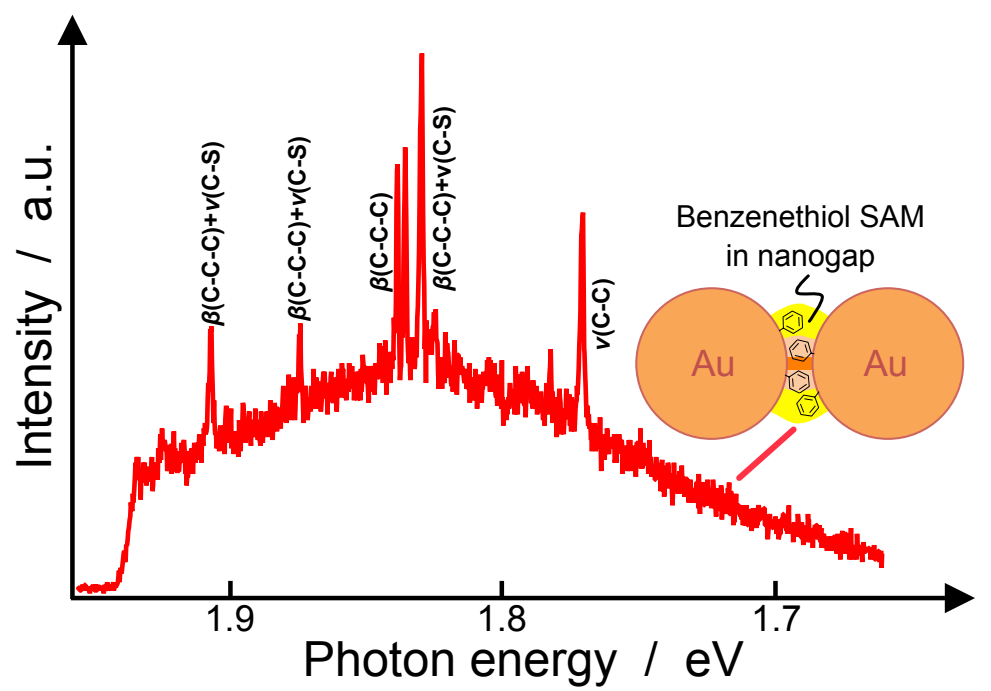

Figure 7.5. The SERS background accompanying spectrum of benzenethiol self-assembled monolayer chemisorbed on gold nanowire array surface.

The fabricated gold nanowire array surface was integrated into a SERS spectroelectrochemical (SEC) analysis system that combines a small volume microfluidic sample chamber on the order of $100 \mu \mathrm{L}$, or less, with a compact three-electrode configuration for in situ surface-enhanced Raman spectro-electrochemistry. This SEC system includes a gold nanowire array surface that serves as the electrochemical working electrode and SERS substrate, a microfabricated $\mathrm{Pt}$ counter electrode, and an external $\mathrm{Ag} / \mathrm{AgCl}$ reference electrode. The gold nanowire working electrode surface enables highly sensitive in situ SERS spectroscopy through large and reproducible SERS enhancements, which eliminates the need for resonant wavelength matching of the laser excitation source with the electronic absorption 
of the target molecule. Hemin, a metalloporphyrin, was used to examine the in situ SERS spectroelectrochemistry, which showed shifts of the oxidation marker band $v_{4}$ with the gold nanowire working electrode surface under potential control.

\subsection{Recommendations}

The gold and silver nanowire array SERS-active surfaces presented in this thesis have been demonstrated to be very effective for surface-enhanced Raman spectroscopy, however, several improvements are required in order to improve the performance and reliability of the surface, which are discussed in the following sections.

\subsubsection{Plasmon substrate development}

Many different techniques have been reported to fabricate metal plasmonic substrates. Colloidal nanoparticle suspensions comprised of bottom-up synthesized metal nanoparticles of various shapes and sizes are attractive due to their preparation simplicity and crystalline structure; however, they typically have poor enhancement reproducibility, which is attributed to many factors including their random composition and lack of precise dimensional control, nanogap spacing, and excitation polarization alignment. Top-down fabricated plasmonic substrates have also been reported extensively over the last three decades. Patterning thin metal layers by using a variety of different nanolithography techniques, such as electron-beam patterning and nanoimprinting, have been used to realize plasmonic surfaces in many different configurations, including discs of many shapes and sizes. Currently, the major challenge in fully exploiting coupled-mode plasmon resonances on metals is the reproducible realization of large area metal nanostructure arrays with uniform sub-20 nm separation nanogaps in high density, as previously described. The fabrication technology described in this thesis provides a step forward in forming large arrays of the nanogaps with small dimensions, however; further technical developments are required in order for the surfaces to be used for surface-enhanced Raman spectroscopy as a general analytical tool.

The problem of adhesion of the thin gold layers to the substrate surfaces in order to prevent delamination after the application of electrode potentials, or long incubation times for the attachment of self-assembled monolayer intermediate layers or molecules that are to be investigated. The poor adhesion of gold to silicon, silicon dioxide, and silicon nitride surfaces is well known, and is usually solved by including a thin adhesion layer, commonly titanium or chromium, before the deposition of the gold layer. However, for thin gold layers, the diffusion of the thin adhesion layer results in strong plasmon damping due to the large absorption of these layers in the visible spectrum. The preliminary results of hemin-modified gold electrode, described in Chapter 6, indicate that the SERS SEC system is a powerful technique 
to monitor surface reactions, which warrants further development. The main limitation of this report is related to the delamination of the gold layer during the attachment of the hemin layer via the 4-mercaptopyridine intermediate layer, which prevented the use of chemical cleaning prior to modifying the electrode surface. Figure 7.6 shows an example of the titanium adhesion plasmon-damping problem. The reflectance spectra in the top row of the Figure 7.6 shows the $Q$-factor decrease as the thickness of the titanium layer is increased.
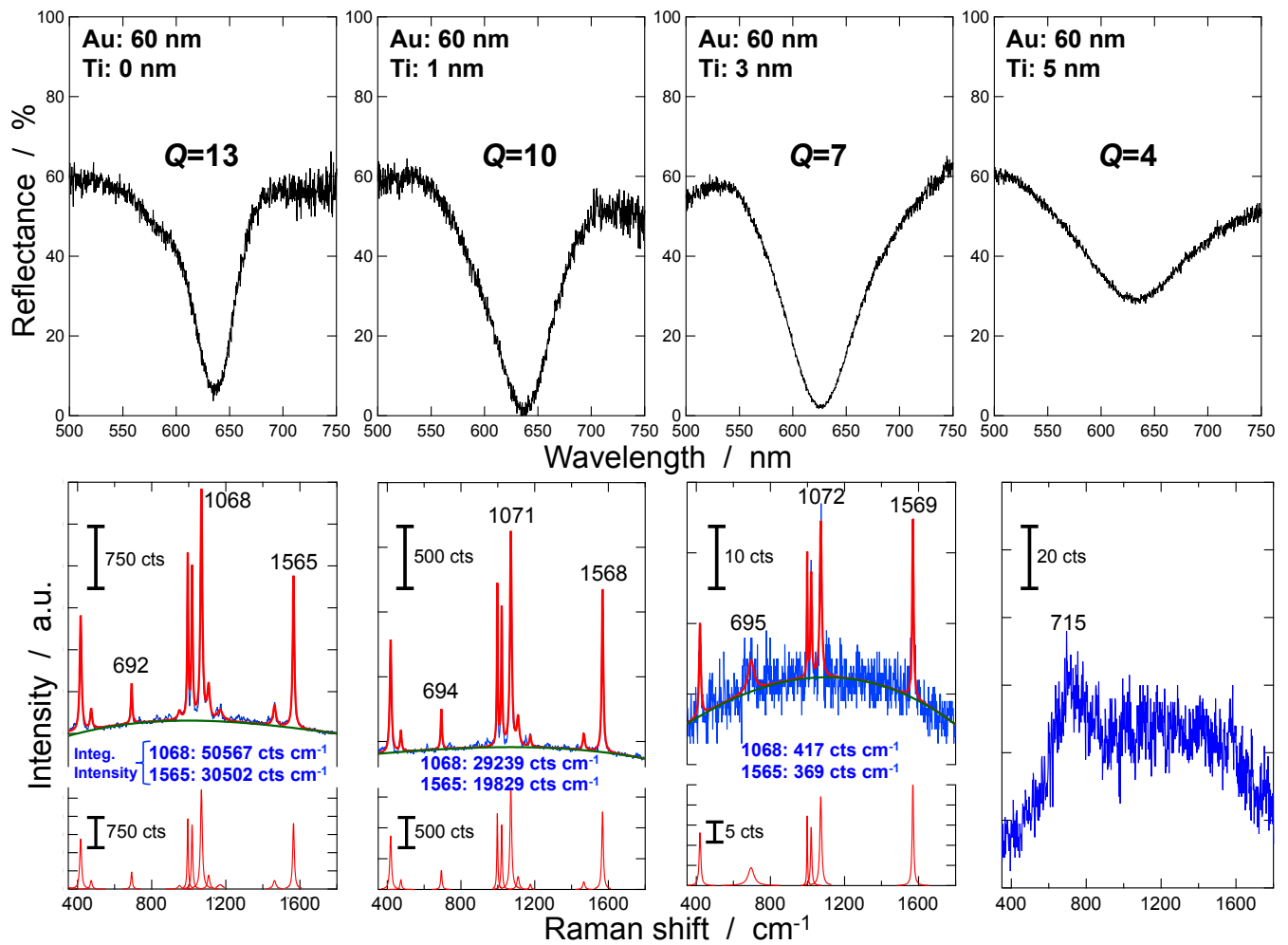

Figure 7.6. The effect of titanium adhesion layers to the localized surface plasmon resonance (top row) and the subsequent SERS spectra from gold nanowire array surfaces modified with benzenethiol self-assembled monolayers.

For titanium layers greater than $5 \mathrm{~nm}$, no localized surface plasmon resonance is observed. The SERS spectra shown in the bottom row of Figure 7.6 shows that the SERS enhancement decreases as the surface plasmon resonance was quenched as the titanium adhesion layer thickness was increased.

\subsubsection{Integration with microfluidic sample chamber}

Microfluidic devices are capable of integrating the sample preparation, reaction, separation, detection and cell culturing, sorting and dissociation into devices with possibly less than $1 \mathrm{~cm}$ microfluidic chips. If the developed metal nanowire array surfaces can be fabricated on glass substrates then the laser excitation of the localized surface plasmon resonance could be moved the bottom of the measurement setup, which will simplify the microscope collection system and more importantly the excitation of the localized surface plasmon resonance energy can be tuned with the incident angle, thus drastically improving the SERS enhancement. With 
these improvements the in situ SERS spectroelectrochemical analysis system can be better integrated with microfluidic devices, which has application in various fields such as catalysis, biochemistry and biophysics. The key to the successful deployment of the SERS analysis system relies on the preparation of a clean and stable SERS-active substrate inside the microfluidic chambers. A next step would be to coupling the SERS analysis system to separation techniques, such as electrophoresis or liquid chromatography to isolate the compounds of interest from the usually complex matrix.

\subsubsection{SERS spectroelectrochemistry}

Surface-enhanced Raman spectroscopy is suitable for pharmaceutical compounds analysis as this technique requires small amount of drug substances and gives rapid chemical identification. Small dose samples of medicine often have substance inhomogeneity over micron scales, therefore, the analysis of such dosage samples by conventional techniques such as mass spectrometry, elemental analysis, spectrophotometry or nuclear magnetic resonance can be very difficult. The small volume SERS spectroelectrochemical system described in this thesis can also be used as a surface spectroscopy investigative tool because a large number of pharmaceutical compounds investigated by SERS are water-soluble.

Furthermore, the integrated SERS spectroelectrochemical system allows combined electrochemistry with surface-enhanced vibrational spectroscopy that can provide novel insights into the interfacial processes of redox proteins that not accessible by other techniques, for example, qualitatively determining surface bonding, conformation and orientation. The in situ SERS spectroelectrochemical system can be applied to investigate solid-liquid, solid-gas and solid-solid interfaces of both fundamental and practical importance for characterization and identification of target molecules.

An interesting application of the in situ SERS SEC system is to investigate and understand the SERS enhancement mechanism and the surface selection rules in both electromagnetic and chemical enhancement that is influenced by changing the applied potential, thus changing the Fermi level of metal as well as dielectric constant of interfacial electrolyte.

Another interesting application is to use an in situ SERS SEC system to study dopamine, which is a hormone and neurotransmitter and plays important roles in the function of the human brain. This is a very interesting molecule as the SERS spectrum in the aromatic ringstretching region with intense bands at $1269 \mathrm{~cm}^{-1}, 1331 \mathrm{~cm}^{-1}, 1424 \mathrm{~cm}^{-1}$ and $1479 \mathrm{~cm}^{-1}$. Since the highest SERS signal of the $1479 \mathrm{~cm}^{-1}$ band, it can be used as the signal for quantitative dopamine measurements in combination with fast-scanning cyclic voltammetry from microscale working electrodes. 


\section{Summary}

Over the last 40 years since its discovery, Surface-enhanced Raman spectroscopy (SERS) has emerged as a powerful analytical technique in various fields of interest such as chemistry, biology and other scientific areas, which provides very large Raman signals of molecules/adsorbates on (or in close proximity to) metal surfaces. SERS is an ultrasensitive detection technique that combines all advances of Raman spectroscopy; for example, provide molecular structural information, as well as surface chemistry of adsorbates on metallic nanostructured surfaces. The widely accepted mechanism of surface-enhanced Raman scattering is electromagnetic mechanism, which is based on extremely large electric field due to highly localized surface plasmon resonances created in metal nanogaps. Making good SERS/plasmonic substrates is a key step toward SERS as a standard analytical technique. Our research group recently reported metallic nanowire array surfaces with tunable sub-20 nm separation nanogaps that are uniform and highly dense hotspot sites over large area (using SEM, AFM and TEM imaging), which was presented in Chapter 3. These substrates were then characterized by using reflectance measurements, which showed that these surfaces are optically uniform and act as very good nanoantennas with which the surface plasmon resonance can be easily and precisely tuned within visible wavelength range of interest; the characterization of these substrates used surface-enhanced Raman spectroscopy was carried out using benzenethiol self-assembled monolayer (SAM) as probed molecules, which showed the reproducible and largely enhanced Raman signal, the average enhancement factor reached up to approximately $10^{7}$, as reported in Chapter 2 and Chapter 4. Another good point for this structure that the silicon nitride templates on silicon wafer created by electron beam lithography, which used for generating the metal nanowire arrays, can be used multiple times by replacing metal thin films (Chapter 3). The developed SERS substrates were applied to single molecules detection of pairs of pyridine/deuterated-pyridine using the bi-analyte method; access to surface conformation characteristics of SAMs, as described in Chapter 2; and studying the well known SERS background continuum, which was described in detail in Chapter 5 with respect to nanostructured gold surfaces and a red laser source. In collaboration with the Analytical Biochemistry Group at the University of Groningen, an integrated microfluidic SERS spectroelectrochemical analysis system for studying Hemin-modified 
electrodes, this small volume SERS spectroelectrochemical cell can further be utilized to access to the orientation of molecules, or monitor surface reaction dynamics and surface conformation, as presented in Chapter 6. Furthermore, it is commonly known that only molecules in the hotspot regions in nanogaps that mainly contribute to the SERS signals, about $1 \%$ of entire surface coverage in this case, the SERS enhancement factor is estimated up to $10^{9}$ when the surface plasmon resonance is tuned to coincide with excitation laser wavelength, this allows regular SERS measurements, not resonant SERS, and therefore these substrates can be used for wider range of molecules of interest and make the measurements more simply, as seen in Chapters 3, 4 and 6. 


\section{Samenvatten}

Sinds de ontdekking van Surface-enhanced Raman spectroscopie / scattering (SERS) in de jaren 70, heeft het zich ontwikkeld tot een zeer krachtige analysetechniek op diverse onderzoeksgebieden, zoals in de chemie en in de biologie. Bij deze techniek worden zeer sterke Raman signalen van moleculen en/of geadsorbeerde verbindingen op of nabij metaaloppervlakken gegenereerd. Het is een uiterst gevoelige detectiemethode die de voordelen van normale Ramanspectroscopie combineert met een hoge signaalopbrengst die het gevolg is van de specifieke structuur van een substraatoppervlak. Een SERS-analyse geeft bijvoorbeeld informatie over de molecuulstructuur of informatie over de oppervlakte chemie van (op nano-structuren) geadsorbeerde verbindingen.

Het algemeen geaccepteerde mechanisme achter de surface-enhanced Raman scattering is een elektromagnetisch effect dat wordt veroorzaakt door een zeer krachtig elektrisch veld. Dit veld is het gevolg van sterk gelokaliseerde surface plasmon resonanties, opgewekt in speciale metalen nano-gaps. Goede SERS/plasmonic substraten zijn essentieel om SERS tot een standaard analysetechniek te kunnen ontwikkelen en het is dus van groot belang om deze substraten te kunnen maken.

Onlangs publiceerde onze onderzoeksgroep over een metaal nano-wire array oppervlak dat bestaat uit afstembare (separatie) nano-gaps van minder dan 20 nanometer. Een dergelijk substraat kan worden gezien als een uniform, over een groot oppervlak verdeelde, verzameling hotspots met een zeer hoge dichtheid (geverifieerd door middel van verschillende technieken als SEM, AFM en TEM). Hierop wordt verder ingaan in hoofdstuk 3. Deze substraten zijn vervolgens gekarakteriseerd door middel van reflectiemetingen. Het resultaat was dat de oppervlakken optisch uniform waren en zeer goed functioneren als nanoantennes. Hiermee kan de surface plasmon resonantie eenvoudig en met grote precisie worden afgestemd op een specifiek golflengtegebied binnen het zichtbare spectrum.

Voor de uiteindelijke karakterisatie van de substraten met behulp van SERS is gebruik gemaakt van benzeenthiol als testmolecuul in de vorm van een self-assembled monolayer (SAM). Uit deze experimenten komt de goede reproduceerbaarheid en de grote versterkingsfactor van het Raman signaal op de geteste substraten naar voren, met een gemiddelde versterkingsfactor die kan oplopen tot ongeveer 10 miljoen, zoals vermeld in 
hoofdstuk 2 en in hoofdstuk 4. De siliciumnitride maskerlaag, die fungeert als template voor de fabricage van de metaal nano-wire arrays wordt gepatroneerd met behulp van elektronenbundellithografie (Electron-beam lithography of kortweg E-beam). Een bijkomend voordeel van deze siliciumnitride template is dat deze laag kan worden hergebruikt. Bij het vervangen van de dunne metaalfilm op het substraat hoeft de nitride template niet opnieuw te worden aangebracht (zie hoofdstuk 3).

De ontwikkelde SERS-substraten zijn gebruikt voor de (enkel molecuul-) detectie van speciale pyridine/gedeuteerde pyridine paren, gebruikmakend van een bi-analiet-methode. Tevens is hierbij gebruik gemaakt van bekende eigenschappen van SAM's, zoals beschreven in hoofdstuk 2. Het welbekende SERS-achtergrondcontinuüm voor met goud bedekte nanostructuren bij toepassing van een rode laser wordt uitgebreid beschreven in hoofdstuk 5 . In samenwerking met de Analytical Biochemistry groep van de Rijksuniversiteit Groningen is een geïntegreerd micro-fluïdisch SERS spectro-elektrochemisch analysesysteem ontwikkeld voor het bestuderen van Hemin-gemodificeerde elektrodes. Deze kleine spectroelektrochemische SERS-cel kan tevens worden gebruikt voor het bepalen van de oriëntatie van moleculen, voor het monitoren van oppervlakte reacties en de verificatie van oppervlaktelagen, zoals besproken in hoofdstuk 6 .

Tenslotte is het algemeen bekend dat alleen moleculen die zich in de zogenaamde hotspotregio's van de nano-gaps bevinden een significante bijdrage leveren aan het SERSsignaal, wat overeen komt met ongeveer $1 \%$ van het totale bedekte oppervlak. De SERS versterkingsfactor kan zelfs oplopen tot circa 1 miljard, als de surface plasmon resonantie zodanig wordt afgestemd dat deze interfereert met de excitatiegolflengte van de laser. Dit maakt de weg vrij voor reguliere SERS-metingen, dus geen SERS resonantie metingen, waardoor de ontwikkelde substraten gebruikt zouden kunnen worden voor een veel grotere groep van moleculen dan tot nu toe. Bovendien zou het de metingen ook een stuk eenvoudiger kunnen maken, zoals vermeld in de hoofdstukken 3, 4 en 6. 


\section{List of publications}

\section{Peer reviewed journal publications}

1. Loan Le Thi Ngoc, Mingliang Jin, Justyna Wiedemair, Albert van den Berg, and Edwin T. Carlen, "Large area metal nanowire arrays with tunable sub-20 nm nanogaps," ACS Nano 7, 5223, 2013.

2. Justyna Wiedemair, Loan Le Thi Ngoc, Albert van den Berg, and Edwin T. Carlen, "Surface-enhanced Raman spectroscopy of self-assembled monolayer conformation and spatial uniformity on silver surfaces," J. Phys. Chem. C 118, 11857, 2014.

3. Tao Yuan, $\dagger$ Loan Le Thi Ngoc, $\dagger$, Mathieu Odijk, Albert van den Berg, Hjalmar Permentier, Rainer Bischoff, and Edwin T. Carlen, "In situ surface-enhanced Raman spectroelectrochemical analysis system with a hemin modified nanostructured gold surface," Anal. Chem. 87, 2588, 2015. († Authors contribute equally to this work)

4. Loan Le Thi Ngoc, Justyna Wiedemair, Albert van den Berg, and Edwin T. Carlen, "Plasmon-modulated photoluminescence from gold nanostructures and its dependence on plasmon resonance, excitation energy, and band structure," Opt. Express 23, 5547, 2015.

5. Loan Le Thi Ngoc, Tao Yuan, Albert van den Berg, Rainer Bischoff, Edwin T. Carlen, "Effects of adhesion layers on surface-enhanced Raman scattering from gold nanostructures," in preparation, February 2015.

\section{International conference proceedings}

1. Mingliang Jin, Loan Le Thi Ngoc, Henk van Wolferen, Herbert Wormeester, Albert van den Berg, and Edwin T. Carlen, Wafer-scale nanogap plasmon resonator substrates for surface enhanced Raman scattering spectroscopy, Proc. $23^{\text {rd }}$ International Conference on Raman Spectroscopy (ICORS 2012), August 2012, Bangalore, India.

2. Justyna Wiedemair, Mingliang Jin, Loan Le Thi Ngoc, Albert van den Berg, and Edwin T. Carlen, Towards the measurement of ${ }^{13} \mathrm{CO}_{2} /{ }^{12} \mathrm{CO}_{2}$ ratios using SERS, Proc. $23^{\text {rd }}$ International Conference on Raman Spectroscopy (ICORS 2012), August 2012, Bangalore, India.

3. Loan Le Thi Ngoc, Mingliang Jin, Albert van den Berg, and Edwin T. Carlen, Large area metal nanowire arrays with submicron pitch and tunable sub-20 nm nanogaps, Proc. The $17^{\text {th }}$ International Conference on Solid-State Sensors, Actuators and Microsystems (Transducers \& Eurosensors XXVII), pp. 206-209, June 2013, Barcelona, Spain. (Poster presentation)

4. Loan Le Thi Ngoc, Albert van den Berg, and Edwin T. Carlen, Towards single molecule surface enhanced Raman scattering, Nanophysics: From Fundamentals to Applications, August 2013, Quy Nhon, Vietnam (Oral presentation). 
5. Loan Le Thi Ngoc, Tao Yuan, Jan van Nieuwkasteele, Mathieu Odijk, Hjalmar Permentier, Ranier Bischoff, Albert van den Berg, Edwin T. Carlen, Surface-enhanced Raman spectroelectrochemical analysis system with metalloporphyrin modified electrodes for drug metabolism investigation, Proc. $25^{\text {th }}$ International Conference on Raman Spectroscopy (ICORS 2014), August, 2014, Jena, Germany (Poster presentation, Student Scholarship Award).

6. Justyna Wiedemair, Mingliang Jin, Loan Le Thi Ngoc, Albert van den Berg, and Edwin T. Carlen, Conformation assessment of self-assembled monolayers using surface-enhanced Raman scattering, Proc. $25^{\text {th }}$ International Conference on Raman Spectroscopy (ICORS 2014), August, 2014, Jena, Germany (Poster presentation).

7. Loan Le Thi Ngoc, Justyna Wiedemair, Albert van den Berg, and Edwin T. Carlen, The contribution of plasmon-enhanced photoluminescence to the SERS background, Proc. SPIE 9163, Plasmonics: Metallic Nanostructures and Their Optical Properties XII, pp. 91632S(1-4), August 2014, San Diego, CA, USA (Poster presentation). 


\section{Acknowledgements}

The research presented in this thesis had been performed in the BIOS Lab-on-a-Chip Group, MESA+ Institute for Nanotechnology, the Faculty of Electrical Engineering, Mathematic and Computer Science, the University of Twente, the Netherlands. This work was completed under the supervision, support, and contribution of my supervisors, colleagues, friends and family. I would like to take this opportunity to thank all of you.

First, I would like to thank Prof. Albert van den Berg for giving me the chance to pursue my doctoral degree in your research group, I am grateful for your supervision and support throughout the last four years. Under your guidance, I found the BIOS Lab-on-a-Chip Group to be a wonderful place to carry out research with great Lab facilities and organization. In addition, I really appreciate all the social activities, such as the BBQ's at Albert's place, mountain biking, and Friday afternoon talks; all of these have been so much fun and have given me many nice memories. I especially enjoyed the Work-Weeks in France and the UK, which gave me the opportunity to visit and learn from excellent research groups abroad; these also gave me the opportunity to learn about different cultures and to form from my colleagues and gain collaboration amongst us.

Second, I'd like to express my gratitude to Prof. Edwin T. Carlen for accepting me as your $\mathrm{PhD}$ student and for being my daily supervisor during last four years. It was a difficult time when I first arrived, but your clear scientific roadmap and strategy have helped me to solve problems step-by-step. Thank you for teaching me many experimental skills in the laboratory and teaching me how to do scientific research. Your passion and patience in doing research has inspired me to explore new challenging scientific topics during my research project. Two years ago, while I was busy preparing my first article, you asked me to come to your office and told me that you were moving to Japan. I was shocked, speechless, and nervous that I would not be able to complete my degree in time. But you promised me that you would continue to supervise my research until graduation. I am now thankful and very glad that we made it to the end with even more articles during the last two years. Thanks for sharing your ideas and helping to bring our research project to life. I very much appreciate to work with you, and wish to continue to learn and collaborate with you in my future career.

Next I want to express my gratitude to the technical team in the BIOS Group. Johan 
Bomer, you gave me my first lessons in the cleanroom and I will never forget the skills I learned from you. You are precise, skillful, and hard working, and help so many students with each chip you fabricate. This is quite opposite with the daily quiet you that we often see in the coffee corner. Jan van Nieuwkasteele, you helped me in so many different ways, such as guiding me in using the microscopes, fixing my laptop, installing software, and helping me build my last experimental setup. Paul ter Braak, thank you for all the help with ordering chemicals and for keeping the chemical lab in order. Sometimes you respond faster than the 'speed of internet'. Thank you Hans for making the shadow mask for my last experiments and Eddy for teaching me how to use the AFM and translating my research summary into Dutch, as well as encouraging me all the time. Thanks to you all for your hard work, dedication, and willingness to help us when we need it - you are an amazing team!

I am thankful to my officemates who made my life much easier and more enjoyable. Thank you so much Arpita for introducing me to the Lab, and providing me so much information and help on how to survive abroad and helping me with so many things. Thanks to Fluer for helping me to adapt to my new workplace as well as taking me to the hospital when I had an eye-infection, and also the very nice dinner that you and Mark organized at your place. Allison, thank you for sharing the office with me for the last four years, and for sharing problems related to research and life, accompanying me during the Work-Week in France and the UK, and being my paranymph. Thanks to Miguel, a very nice and courageous man who dared to share an office with all ladies. Thanks to Wei-Shu for making very tasty chicken soup for me, the dinners at your place, and helping me with things during my absence from BIOS for the last few months. Thanks also to Henriette, Rosa, Liza and Movaffag. All of you helped to make my life in the office full of fun and laughter, and help me to get through the difficult times and failed experiments.

I sincerely acknowledge Jan Eijkel, Severine Le Gac, Wouter Olthuis and Loes Segerink for all your encouragement, support and discussions during the time I have been in the group. I would like to thank Mathieu Odijk for the fruitful collaboration between BIOS and the Analytical Biochemistry Group at the University of Groningen during the last year of my project, and for teaching me some of the basic fundamentals of electrochemistry; hopefully we can continue to collaborate in the future. Thanks a lot to Wesley for depositing nitride layers on my wafers, learning together to use the AFM and Raman microscope, and being my paranymph. I would like to acknowledge Mingliang Jin and Justyna Wiedemair for training me in the early stage of my project. I also want to take this opportunity to thank Hermine for all the paper work that you have helped with over the last four years. Thanks to Yawar, 
Mathieu and Arpita who helped make a wonderful trip to the conference in Barcelona. I'd like to thank all wonderful colleagues who organized and participants of the many activities in the group, such as the Monday morning talks, technical discussions, coffee breaks, dinners, and concerts at the city center: Lennart, Verena, JP, Jean-Baptiste, Floris, Stefan, Burcu, Marinke, Laura, Afshan, Trieu, Hai, Susan, Rogier, Miguel, Tao, Bjorn, Ashish, Songyue, Rik, Adithya, Sertan, Lenka, Maria, Anna, Martijn, Paul, Ray, Yanbo, Zhenxia, Lingling, Rerngchai, Masood; you all made BIOS a wonderful place to work.

I am thankful to all my Vietnamese friends in Enschede and Europe. Special thanks to Anh Minh-Chi Giang family (including Dung-Manh), Anh Hanh-Lan Anh (including Tom-Tep), Tan-Dao, Tuan-Hieu families who were always willing to take care of my daughter - Phuong Anh, and help when we needed it. Thanks also to Anh Hien, So, Duc, Trung, Yen, Hieu, Phong, Bach, Tram, Chau, Viet Anh, Quyen, Van, Dung, Hanh, Trang, Tuan, Hang, Son, Trinh, Duc-Hong family for making delicious food, cakes and lots of fun at BBQs and our new year celebrations and mid-autumn festivals. Thank you to my colleagues from Quy Nhon University and ITIMS: Hai-Trung, Hung-Hang, Hieu (Belgium), Hien, Anh, Tan, Huong, Thu (Germany), Thuong (Italy) for warmly welcoming us when we visited; special thanks to Bay, Vinh, Tam-Thuan, An-Ha, Thang who travelled many times to visit us in Enschede. We had so much fun and it made us less homesick. I am grateful to Prof. Jurriaan Schmitz for introducing me to the BIOS group, Alexey Kovalgin and Michel de Jong for your understanding and making the most convenient place for us to work and take care of Phuong Anh. Thanks to Hao's officemates and colleagues for organizing nice dinners and drinks. Special thanks to Tom-Elly for very nice dinners and memories at your place and our places in Enschede and Den Haag. Thanks to my teachers and colleagues at the Physics department, Quy Nhon University and ITIMS who supported and encouraged me during the last four years. Thank you very much to all the teachers and parents at the International School in Enschede and Den Haag for teaching and caring for my daughter. I apologize if I missed mentioning anyone.

Last but not least, I'd like to give special thanks to my husband, Hao van Bui, for arranging everything before Phuong Anh and I arrived in the Netherlands, and for taking care of our lovely daughter - We are very happy to see how much Phuong Anh has grown. Thank you so much to my Angel, Phuong Anh, who always puts smile on my face, a hug and smile from you helped me to forget any problems and restore my energy after a long day of experiments. Stories from your school always brought more flavor and fun to our every dinner - Love you so much! I want to express my gratitude to my parents who raised me and 
give me endless support. Thanks to my parents in law, my sisters, sisters and brothers in law, my grandparents, my uncles, my aunts who support and encourage me over the last four years.

Finally, I would like to express my gratitude to The Ministry of Education and Training, Vietnam (MOET), Project No. 322 for funding my doctoral program. Thanks to Ms. Tran Thi Nga and Ms. Nguyen Hong Hanh for all the paper work and consultation over the last four years.

Thanks to all of you for making this part of my life unforgettable!

Cheers,

Loan 